Pacific Northwest National Laboratory

Operated by Battelle for the

U.S. Department of Energy

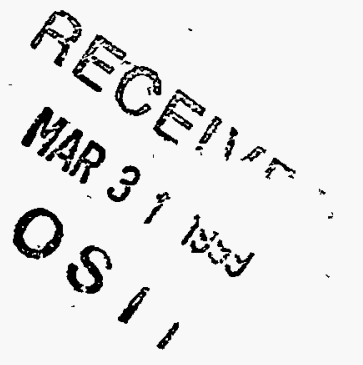

\title{
Borehole Data Package for Well 299-E33-44 at Single-Shell Tank Waste Management Area B-BX-BY
}

D. G. Horton

S. M. Narbutovskih

March 1999

Prepared for the U.S. Department of Energy under Contract DE-ACO6-76RLO 1830 


\section{DISCLAIMER}

This report was prepared as an account of work sponsored by an agency of the United States Government. Reference herein to any specific commercial product, process, or service by trade name, trademark, manufacturer, or otherwise does not necessarily constitute or imply its endorsement, recommendation, or favoring by the United States Government or any agency thereof, or Battelle. Memorial Institute.

\section{PACIFIC NORTHWEST NATIONAL LABORATORY operated by \\ BATTELLE \\ for the \\ UNITED STATES DEPARTMENT OF ENERGY}

under Contract DE-AC06-76RLO.1830

Printed in the United States of America

Available to DOE and DOE contractors from the

Office of Scientific and Technical Information, P.0. Box 62, Oak Ridge, TN 37831;

prices available from (615) 576-8401.

Available to the public from the National Technical Information Service, U.S. Department of Commerce, 5285 Port Royal Rd., Springfield, VA 22161 


\section{DISCLAIMER}

This report was prepared as an account of work sponsored by an agency of the United States Government. Neither the United States Government nor any agency thereof, nor any of their employees, make any warranty, express or implied, or assumes any legal liability or responsibility for the accuracy, completeness, or usefulness of any information, apparatus, product, or process disclosed, or represents that its use would not infringe privately owned rights. Reference herein to any specific commercial product, process, or service by trade name, trademark, manufacturer, or otherwise does not necessarily constitute or imply its endorsement, recommendation, or favoring by the United States Government or any agency thereof. The views and

opinions of authors expressed herein do not necessarily state or reflect those of the United States Government or any agency thereof. 


\section{DISCLAIMER}

Portions of this document may be illegible in electronic image products. Images are produced from the best available original document. 


\section{Borehole Data Package for Well 299-E33-44 at Single-Shell Tank Waste Management Area B-BX-BY}

D. G. Horton

S. M. Narbutovskih

March 1999

Prepared for the U.S. Department of Energy under Contract DE-AC06-76RLO 1830

Pacific Northwest National Laboratory

Richland, Washington 99352 



\section{Acknowledgment}

The analyses of particle size distribution, moisture content, $\mathrm{pH}$, electrical conductivity, cation exchange capacity, calcium carbonate content, and alkalinity were done in the Applied Geology and Geochemistry Group laboratory, Pacific Northwest National Laboratory, Richland, Washington. The work was supervised by Jeff Serne. His laboratory also produced the 1:1 water:sediment extracts for major cation and anion analyses. The cation analyses were done at the Chemical Analysis Laboratory at the University of Georgia in Athens, Georgia. The anion analyses were done in the Pacific Northwest National Laboratory interfacial geochemistry laboratory. Mr. Serne provided interpretations of all the physical and chemical data from sediment samples, and those interpretations are incorporated into this report. His contribution is very much appreciated. 



\section{Contents}

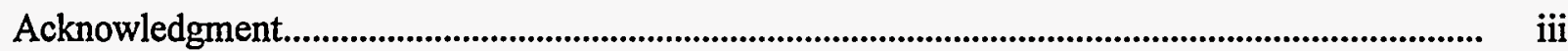

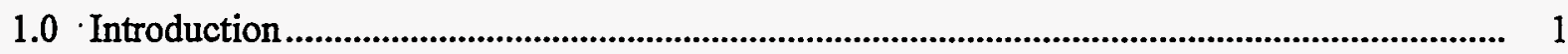

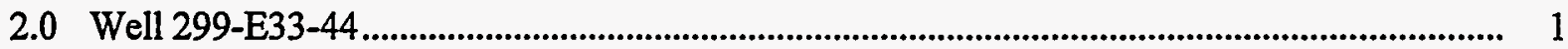

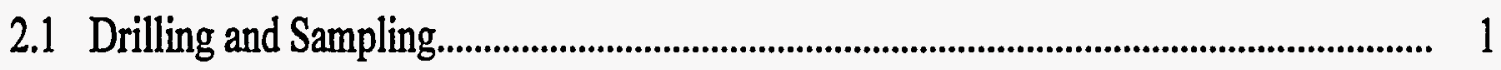

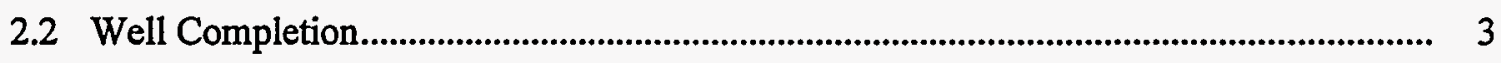

2.3 Well Development and Pump Installation ......................................................................... 4

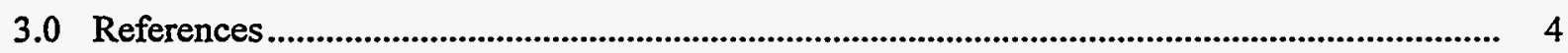

Appendix A - Well Construction and Completion Documentation................................................ A.1

Appendix B - Physical and Chemical Properties Data ................................................................... B.1

Appendix C - Geophysical Logs .......................................................................................... C.1

\section{Figure}

1 Map of Waste Management Area B-BX-BY and Location of Wells in the Groundwater Monitoring Network

\section{Table}

1 Survey Data for Well 299-E33-44 


\subsection{Introduction}

One new Resource Conservation and Recovery Act (RCRA) groundwater monitoring well was installed during September 1998 at the single-shell tank farm Waste Management Area (WMA) B-BXBY in 1998 in fulfillment of Tri-Party Agreement (Ecology 1996) milestone M-24-40. The well is 299E33-44 and is located east of the BY single-shell tank farm. The well is a new upgradient monitoring well drilled in support of the groundwater assessment program at WMA B-BX-BY. The locations of all wells in the monitoring network at WMA B-BX-BY are shown on Figure 1.

The groundwater monitoring plan for single-shell tanks (Caggiano and Goodwin 1991) describes the hydrogeology of the 200 East Area and WMA B-BX-BY. An Interim Change Notice to the groundwater monitoring plan provides justification for the new well. The new well was constructed to the specifications and requirements described in Washington Administrative Code (WAC) 173-160 and WAC-173-303.

This document is a compilation of information on the drilling and construction, well development, pump installation, and sediment testing and analyses applicable to well 299-E33-44. Appendix A contains copies of the geologist's log, the Well Construction Summary Report, and Well Summary Sheet (as-built diagram); Appendix B contains results of laboratory analyses completed on samples of sediment from the well; and Appendix $\mathrm{C}$ contains geophysical logs. An aquifer test (slug test) was done in the well after well completion. Results from the aquifer test will be published elsewhere. Additional documentation concerning well construction is on file with Bechtel Hanford, Inc., Richland, Washington.

English units are used in this report because they are used by drillers to measure and report depths and well construction details. The conversion is made by multiplying feet by 0.3048 to obtain meters or multiplying inches by 2.54 to obtain centimeters.

\subsection{Well 299-E33-44}

\subsection{Drilling and Sampling}

Well 299-E33-44 was drilled using a cable tool rig and a drive barrel from 0 to $247 \mathrm{ft}$ below ground surface (bgs) and cable tool rig and hard tool from 247 to $255 \mathrm{ft}$ bgs. The well was drilled to a total depth of $255 \mathrm{ft}$ bgs during September 1998. Temporary 8 5/8-in.-outside-diameter, carbon steel casing was used from ground surface to $247.8 \mathrm{ft}$ bgs. Approximately 45 gal of water was added to the borehole during hard tool drilling in basalt from 247 to $255 \mathrm{ft}$ bgs. Static water level was $239.36 \mathrm{ft}$ bgs on September 26, 1998. 


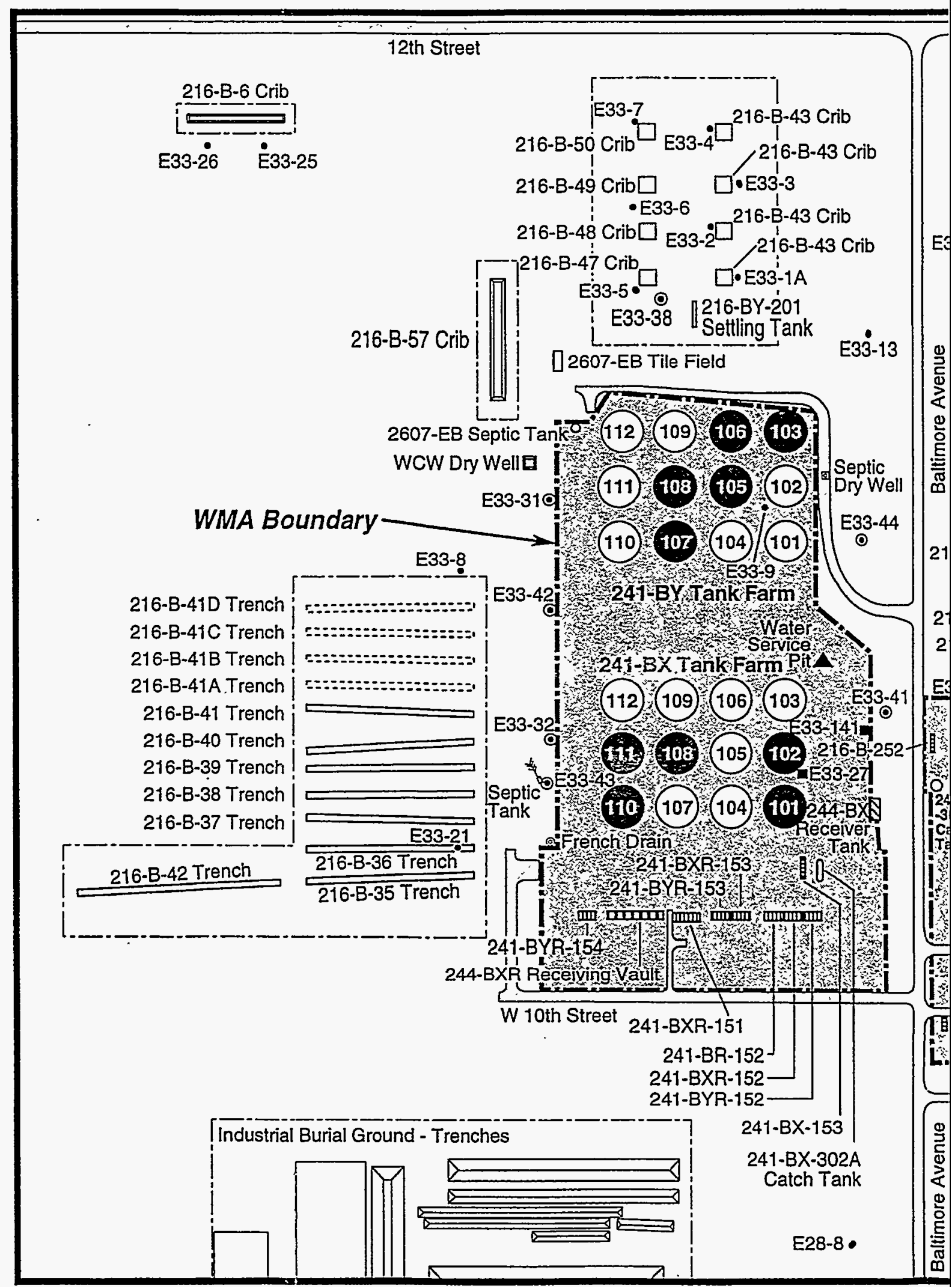

Figure 1. Map of Waste Management Area B-BX-BY an Monitoring Network 
Sediments encountered during drilling are part of the Hanford formation and were predominantly sandy gravel and sand with minor silty sand and silty, sandy gravel from the surface to about $163 \mathrm{ft}$ bgs; silt and sandy silt from 163 to about $174 \mathrm{ft}$ bgs; gravelly sand and silty, sandy gravel from 174 to $247 \mathrm{ft}$ bgs; and basalt from $247 \mathrm{ft}$ to total depth. A geologist's log is included in Appendix A.

Grab samples were collected at 5- $\mathrm{ft}$ intervals from the surface to $230 \mathrm{ft}$ bgs for analysis of leachable ions, $\mathrm{pH}$, electrical conductivity, cation exchange capacity, and moisture and calcium carbonate contents. Additional grab samples were collected at 70, 125, 230, 231, 234, 236, 241, 243, and $245 \mathrm{ft}$ bgs for analysis of particle size distribution. One split spoon sample was collected from 208 to $210.5 \mathrm{ft}$ bgs ( $100 \%$ of recovery) for determination of particle size distribution, moisture content and hydraulic properties. Samples also were collected every foot from $230 \mathrm{ft}$ to $246 \mathrm{ft}$ bgs for analysis of residual contaminants left from a falling water table. Results from all available laboratory analyses are in Appendix B. At depths from which samples were not collected for other purposes, sediment samples were collected for geologic description and archive at approximately 5-ft intervals throughout the entire borehole.

The particle size data show that the sediment at 70 to $74 \mathrm{ft}$ bgs is very fine grained (predominantly very fine sand, silt and clay), the sediment at $125 \mathrm{ft}$ bgs is coarser sand, and the sample from near $240 \mathrm{ft}$ bgs is a mixture of gravel, sand, and silt. There is a thin layer of cemented material at $166 \mathrm{ft}$ depth that appears to be sulfate rich (possibly gypsum). The moisture contents seem to correlate with particle size with the finer grained material having higher moisture content.

The water extract nitrate data for depths 80 to $105 \mathrm{ft}$ and 140 to $175 \mathrm{ft}$ seem higher than natural sediment, suggesting that the vadose zone in this area may have been impacted by liquid discharges. Sulfate is higher than expected in many of the same samples. The cation exchange capacity seems to show a positive correlation with particle size distribution as is expected. The mix of divalent and monovalent cations is within the range expected for natural sediments.

The borehole and drill cuttings were monitored regularly for organic vapors and radionuclide contaminants. No contamination was found.

A gross gamma-ray log, using a sodium iodide detector, and a neutron moisture log were obtained for lithologic purposes on September 23, 1998. A high resolution, spectral gamma-ray log was obtained on September 24, 1998, for identification of man-made radionuclides. All geophysical logs were run from 0 to $254 \mathrm{ft}$ bgs. The spectral gamma-ray log identified cesium- 137 near the surface ( 0.5 to $3 \mathrm{ft})$ with a maximum activity of $3 \mathrm{pCi} / \mathrm{g}$ and cesium-137 at intermittent depths throughout the borehole at activities $<0.3 \mathrm{pCi} / \mathrm{g}$. No other man-made radionuclides were identified. All geophysical logs are in Appendix C.

\subsection{Well Completion}

The permanent casing and screen were installed in well 299-E33-44 during September 1998. A 4-in.inner-diameter, stainless steel, wire wrap (0.01 in. slot) screen was set from 253.0 to $238.0 \mathrm{ft}$ bgs. The 
permanent casing is 4-in.-inner-diameter stainless steel from $238.0 \mathrm{ft}$ bgs to $1.94 \mathrm{ft}$ above ground surface. Centralizers were placed above and below the screen and every $40 \mathrm{ft}$ from the screen to the surface. The bottom of the screen has a 4-in. end cap.

The sand pack is 20 to 40 mesh silica sand from 253.9 to $227.2 \mathrm{ft}$ bgs. The annular seal is $0.25 \mathrm{in}$. bentonite pellets from 227.2 to $199 \mathrm{ft}$ bgs, medium bentonite chunks from 199 to $9.8 \mathrm{ft}$ bgs, and Portland cement from $9.8 \mathrm{ft}$ to the surface. $\mathrm{A} 4 \mathrm{ft}$ by $4 \mathrm{ft}$ by $6 \mathrm{in}$. concrete pad was placed around the well at the surface. A protective casing with locking cap, four protective steel posts, an a brass marker stamped with the well number were set into the concrete pad. The Well Construction Summary Report and the Well Summary Sheet (as-built) are in Appendix A.

The vertical and horizontal coordinates of the well were surveyed in December 1998. The horizontal position of the well was determined by Global Positioning System observations referenced to horizontal control stations established by the U.S. Army Corps of Engineers. The coordinates are Washington Coordinate System, South Zone, NAD83/91 datum. Vertical datum is NAVD 1988 and is based on existing bench marks established by the U.S. Army Corps of Engineers. Survey data are included in Table 1 .

Table 1. Survey Data for Well 299-E33-44

\begin{tabular}{|l|c|c|c|l|}
\hline \multirow{3}{*}{$299-$ Well Name } & Easting (m) & Northing (m) & Elevation (m) & \\
\hline & $573,706.411$ & $137,469.1635$ & & Center of casing \\
\cline { 2 - 5 } & & & 196.7733 & "X" on casing rim \\
\cline { 2 - 5 } & $573,706.420$ & $137,469.4315$ & 196.0284 & Brass cap \\
\hline
\end{tabular}

\subsection{Well Development and Pump Installation}

Well 299-E33-44 was developed on October 1, 1988. A 2 hp Grundfos pump was used to remove about $300 \mathrm{gal}$ of formation water from the well at $4 \mathrm{gal} / \mathrm{min}$. The final turbidity was $3.23 \mathrm{NTU}$.

A dedicated Hydrostar sampling pump was installed in well 299-E33-44 on October 17, 1998. The sampling pump intake is at $251.4 \mathrm{ft}$ depth relative to the brass cap (see Table 1 ).

\subsection{References}

Caggiano, J. A., and S. M. Goodwin. 1991. Interim Status Groundwater Monitoring Plan for the SingleShell Tanks. WHC-SD-EN-AP-012, Rev. 1. Westinghouse Hanford Company, Richland, Washington. 
Ecology - Washington State Department of Ecology, U.S. Environmental Protection Agency, and U.S. Department of Energy. 1996. Hanford Federal Facility Agreement and Consent Order. Document No. 89-10, Rev. 4 (The Tri-Party Agreement), Ecology, Olympia, Washington.

RCRA - Resource Conservation and Recovery Act. 1976. Public Law 94-580, as amended, 90 Stat. 2795,42 USC 6901 et seq.

WAC 173-160, Washington Administrative Code. Minimum Standards for Construction and Maintenance of Wells. Olympia, Washington.

WAC 173-303, Washington Administrative Code. Dangerous Waste Regulations. Olympia, Washington. 
Appendix A

Well Construction and Completion Documentation 


\section{WELL CONSTFUCTION SUMMARY REPOR ,}

$\begin{array}{ll}\text { Start Date: } & 9 / 12 / 98 \\ \text { Finish Date: } & 9 / 26 / 98\end{array}$ Page 1 of 1

\begin{tabular}{|c|c|c|c|c|}
\hline 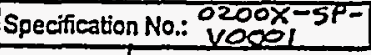 & \multirow{2}{*}{\multicolumn{2}{|c|}{ Rev. No.: 0}} & Well Name: $299-E 33-44$ & Fonfinto: 88554 \\
\hline ECNs: $N A$ & & & \multicolumn{2}{|c|}{ Approximate Location: 100 'Eost of $241-B Y$ Fankfarm, $200 \mathrm{E}$} \\
\hline Project: 1998 RCRA & \multicolumn{2}{|l|}{9 Drilling } & \multicolumn{2}{|c|}{ Other Companies: $\mathrm{Cff} 2 \mathrm{mH}$ Hill } \\
\hline Dilling Company: Lqyene & \multicolumn{2}{|c|}{ Christensen } & \multirow{2}{*}{\multicolumn{2}{|c|}{ Geologist(s): D.C.Weetes }} \\
\hline \multicolumn{3}{|l|}{ Driller. M. Ulngspir } & & \\
\hline \multicolumn{3}{|c|}{ TEMPORARY CASING AND DRULL DEPTH } & \multicolumn{2}{|c|}{ DRILLING METHODHOLEDLAMETER: } \\
\hline "SizellGradellbs. Per Ft." & Interval & Shoe O.Dת.D. & \multicolumn{2}{|r|}{ Diameter From. } \\
\hline \multirow[t]{5}{*}{ Canbonsteel (FJ) } & $0^{\prime}-247.8^{\circ}$ & $0.8 \% 0.66^{\prime}$ & Cable Tool: $0180-244^{\prime}$ & Diameler From $0^{\circ}$ to $255^{\prime}$ \\
\hline & $=$ & & Air Rotary: & Diameter From \\
\hline & $=-$ & & A.R. w/Sonic: & Diameler From \\
\hline & $=$ & & & Diameter From \\
\hline & $\therefore$ & & & Diameter From \\
\hline \multicolumn{3}{|c|}{ Indicate Welded (M) - Flush Joint (FJ) Coupled (C) \& Thread Design } & & Diameter From \\
\hline \multirow{2}{*}{\multicolumn{3}{|c|}{. }} & & \\
\hline & & & \multicolumn{2}{|l|}{ Drilling Fluid: Water } \\
\hline Total Drilled Depth: $255^{\prime}$ & Hole Dia @ TD: & 8" & \multirow{2}{*}{\multicolumn{2}{|c|}{\begin{tabular}{|l|} 
Total Amt of Water Added During Drilling: $4599 /$ during A7 \\
Static Water Level: 239.36 Date: $9 / 26 / 98$ \\
\end{tabular}}} \\
\hline \multicolumn{3}{|c|}{ Well Straightness Test Results: $N A$} & & \\
\hline
\end{tabular}

\begin{tabular}{|c|c|c|c|c|c|}
\hline Sondes (type) & Interval & Dato & Sondes (type) & Interval & Dats \\
\hline $\mathrm{NaI}$ (Grossgamma) & $0^{\prime}-254^{\prime}$ & $9 / 23 / 98$ & & & \\
\hline Neutron & $0^{\prime} .254$ & $9 / 23 / 98$ & & $=$ & \\
\hline Spectral Grmmalk-uT & $0^{\prime}-254$ & $9 / 24 / 98$ & & $=$ & \\
\hline
\end{tabular}

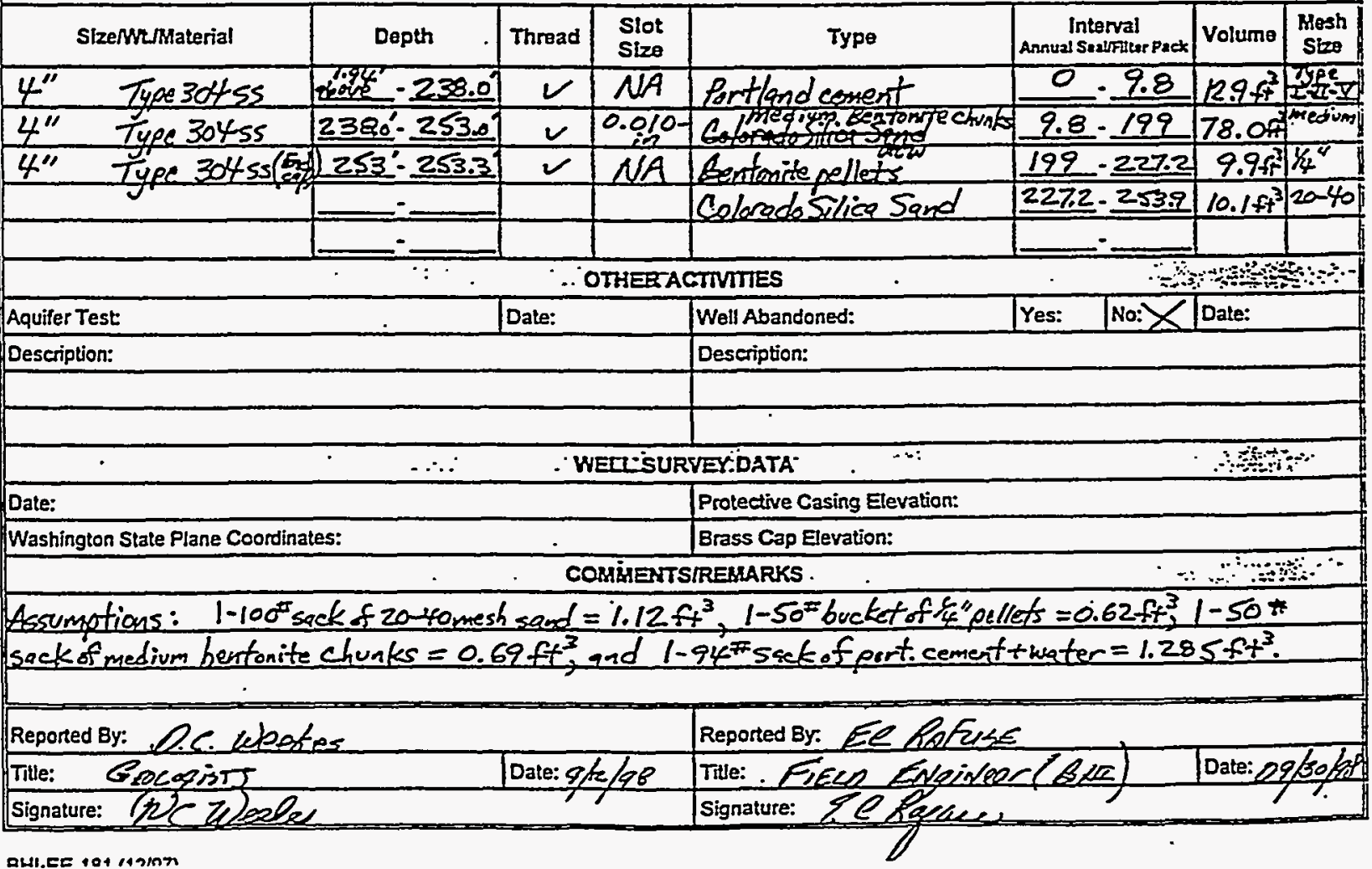




\begin{tabular}{|c|c|c|c|c|}
\hline \multicolumn{4}{|c|}{ WELL SUMMARY SHEET } & $\begin{array}{l}\text { Page } 1 \text { of } 1 \\
\text { Date: } 9 / 26 / 98\end{array}$ \\
\hline \multicolumn{2}{|l|}{ Well 1D: $\quad B 8554$} & \multicolumn{3}{|c|}{ Well Name: $299-E 33-44$} \\
\hline \multicolumn{2}{|c|}{ Location: $100^{\prime}$ Eqst of $241-T Y$ Tan kfarm, $200 \mathrm{E}$} & \multicolumn{3}{|c|}{ Project: 1998 RCRA Drilling } \\
\hline Prepared By: DCWeekes & Date: $9 / 26 / 98$ & Reviewed B & By: & Date: og/2g/as \\
\hline \multicolumn{2}{|l|}{ signature: $A^{\prime C}$ Zlee/zer } & \multicolumn{3}{|c|}{ Signature: Ĺluesh Latues } \\
\hline \multicolumn{2}{|l|}{ CONSTRUCTION DATA } & \multirow{2}{*}{$\begin{array}{c}\text { Depth in } \\
\text { Feet }\end{array}$} & \multicolumn{2}{|c|}{ GEOLGEICIHYDROLOGIC DATA } \\
\hline Description & Diagram & & $\begin{array}{c}\text { Graphic } \\
\text { Log }\end{array}$ & Lithologic Description \\
\hline Portland Cement $0-9.8^{\prime}$ & \multirow{25}{*}{ 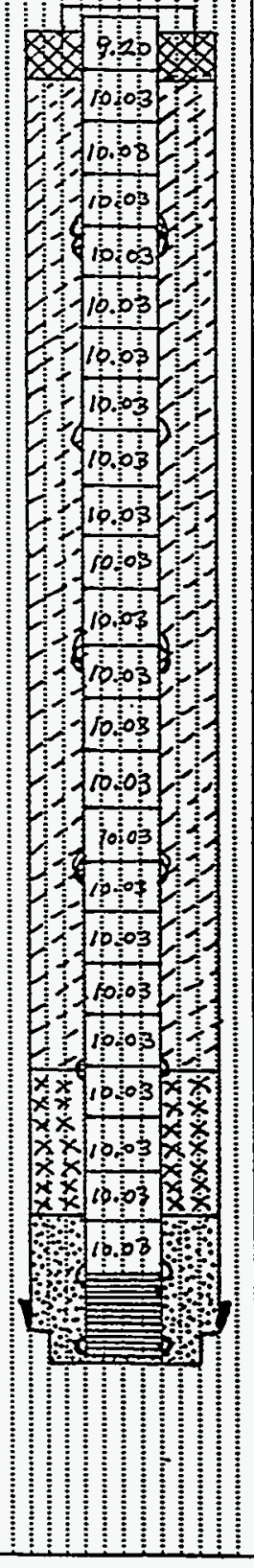 } & \multirow{25}{*}{$150-$} & & O'O': Siltu Sindy GRAVEL \\
\hline Med.chunk Bentonite $9.8^{\prime}=199^{\prime}$ & & & & \\
\hline "s"Bentonite Bellets 199'-227.2' & & & & 9'23: Sandy GRAVEL \\
\hline Colorado Silica Sand(20.40) & & & & 23'-39': Slightly Sitty Gravelly SAND \\
\hline from $227.2^{\circ}-253.9^{\circ}$ & & & & $39^{\prime}-44^{\prime}:$ Gravelly SAND \\
\hline & & & & $\Psi 4^{\prime}>0^{\prime}: \operatorname{SAND}$ \\
\hline $4 " 10$ Type 304 stainless steel & & & & $70^{\prime}-745^{\prime}:$ silty SAND \\
\hline (flush joint threaded) $1.9 x^{\prime}$ bove & & & & $74.5^{\prime}-124^{\prime}:$ SAND \\
\hline ground to 238.0 below graund & & & & \\
\hline $4^{\prime \prime} 1 D$ Type $30455 \cdot 0.010$-in & & & & \multirow[t]{2}{*}{ 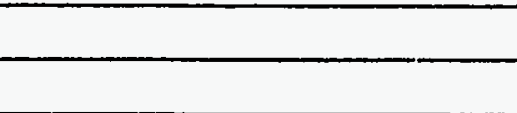 } \\
\hline slot continuous wire wand & & & & \\
\hline sreen from $238.0^{\prime}-253.0^{\prime}$ & & & & $124^{\prime}-128:$ Silty Sandy GRAVEL \\
\hline 55 end cap $253.0^{\prime}-253.3^{\prime}$ & & & & $128^{\circ}-134^{\circ}$ : Slightly Sitty SAND \\
\hline & & & & $134^{\prime}-154^{\prime}:$ SAND \\
\hline Centralizers (ss) above and & & & & $154^{\prime}-163.5^{\prime}:$ Slighthy Silty SAND \\
\hline below the screen and at & & & & $163.5^{\prime}-165^{\prime}:$ Silty SAND \\
\hline $40 \mathrm{ft}$ intervals on the riser. & & & & $165^{\prime}-167.5^{\prime}:$ Sandy SlLT \\
\hline & & & & $167.5^{\prime}-174.5^{\prime}:$ SAND \\
\hline & & & & 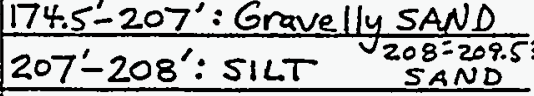 \\
\hline & & & & $20 \% .5^{\prime}-212.5^{\prime}:$ S1LtY Sqndy GRAVE \\
\hline Water level $239.36^{\prime}(9 / 26 / 98)$ & & & 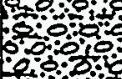 & $2229^{\prime}-231:$ Gravelly silty SAND \\
\hline & & & & $231^{\prime}-247.4:$ Sitty Sendy GAAVEL \\
\hline All depths are fromground surf. & & & & $247.4^{\circ} 255^{\prime}:$ BASAL \\
\hline casingremoved & & & & \\
\hline from the ground & & & & \\
\hline
\end{tabular}

BHI-EE-189 (12/97) 


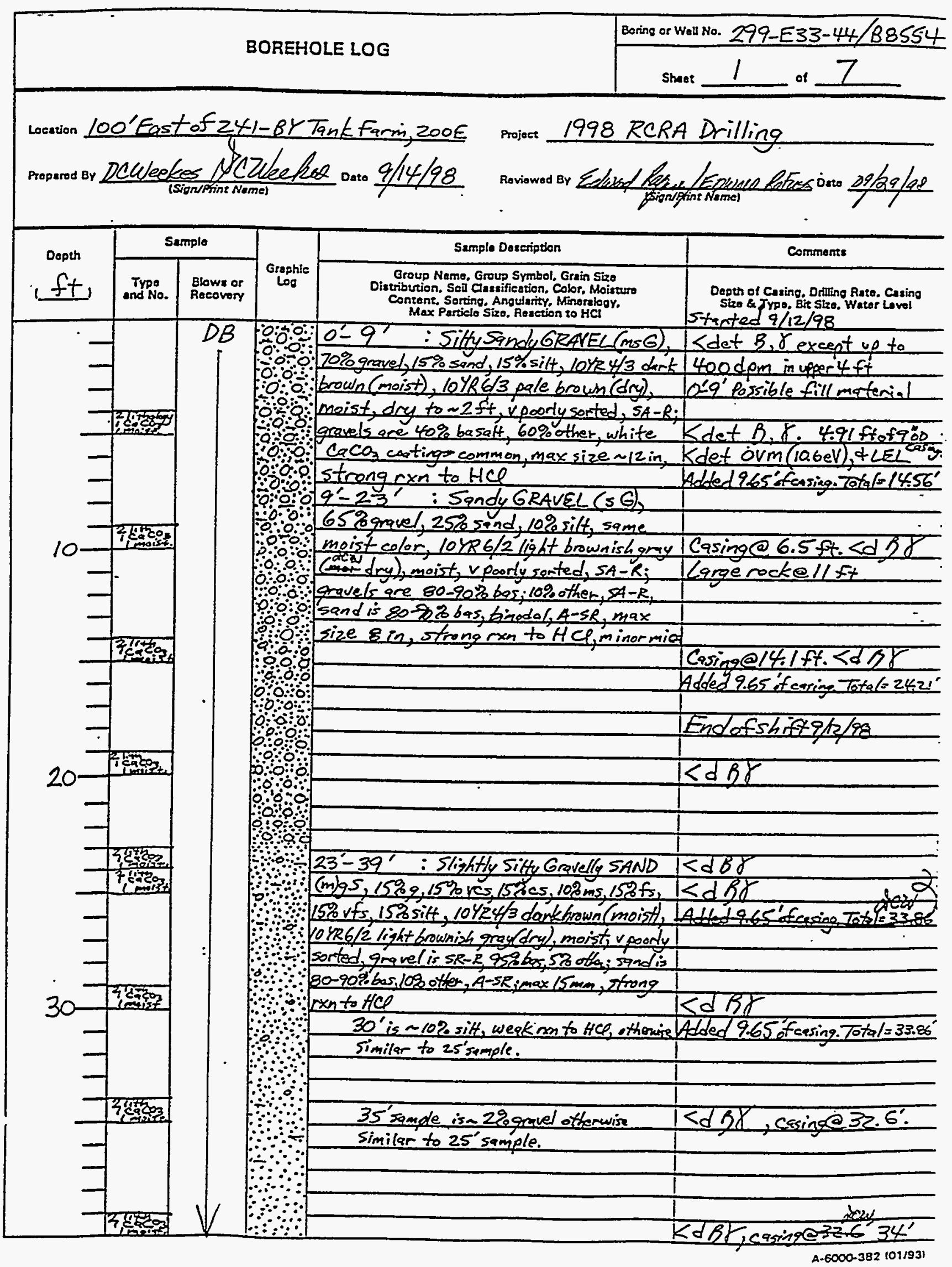




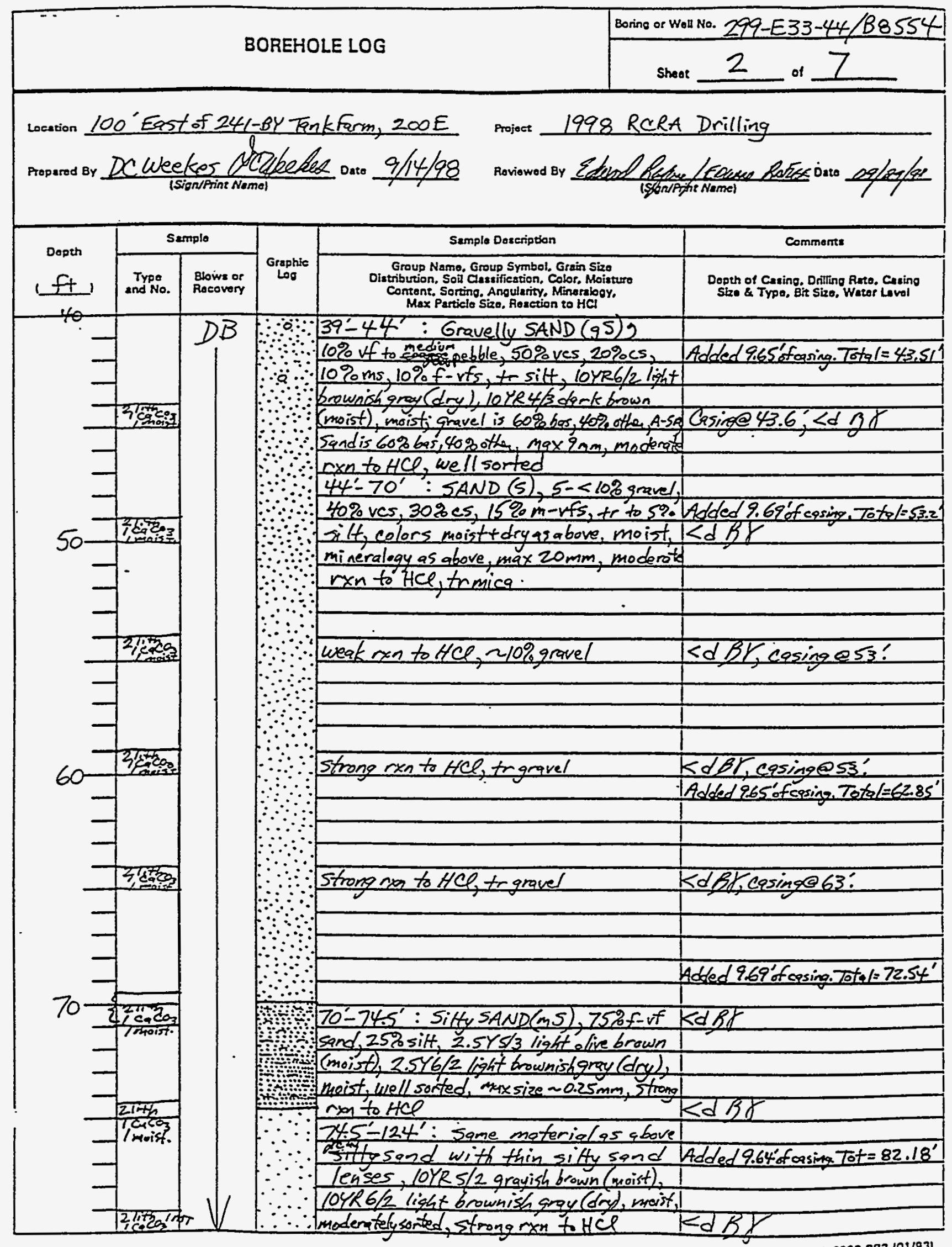




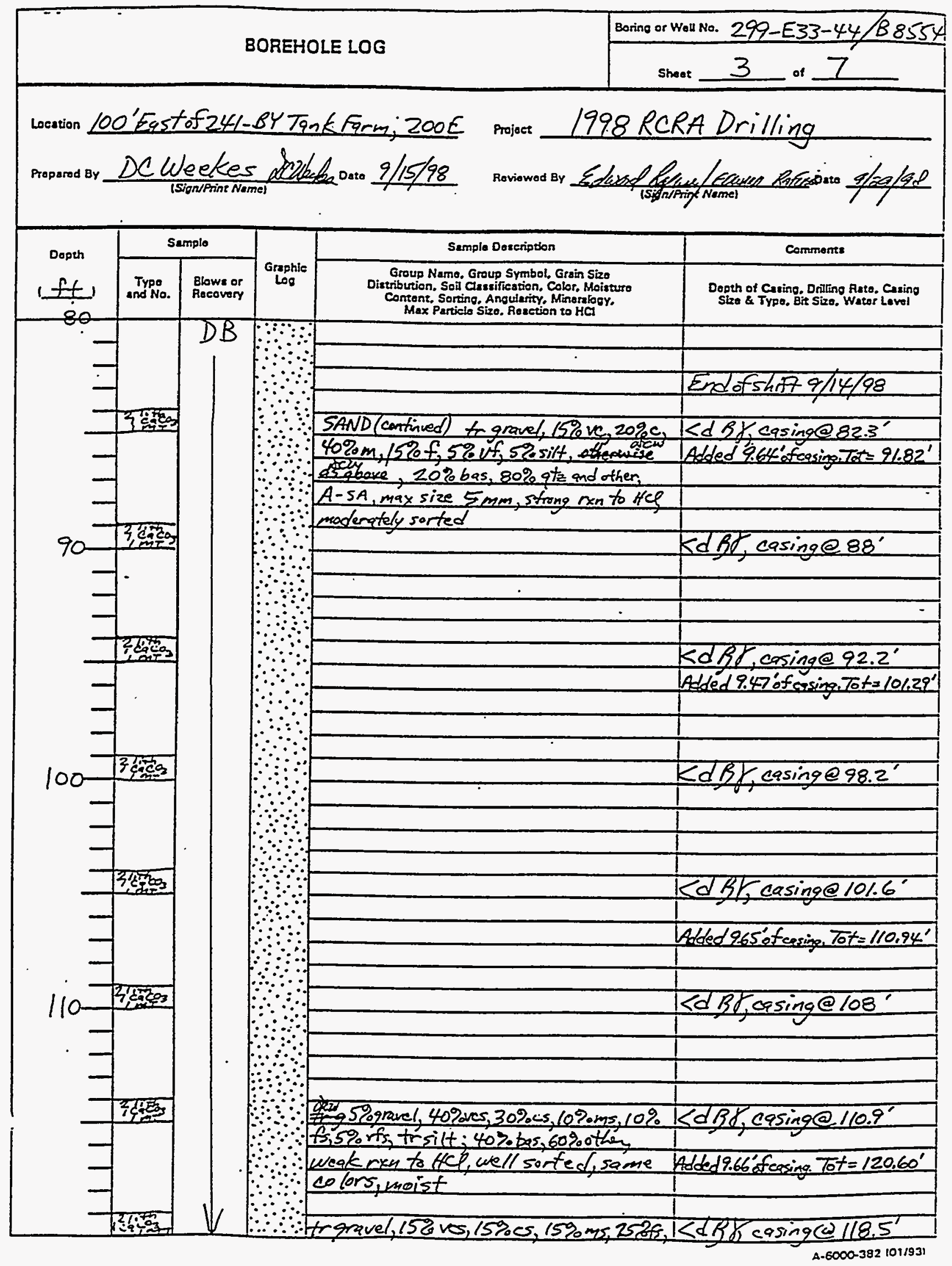




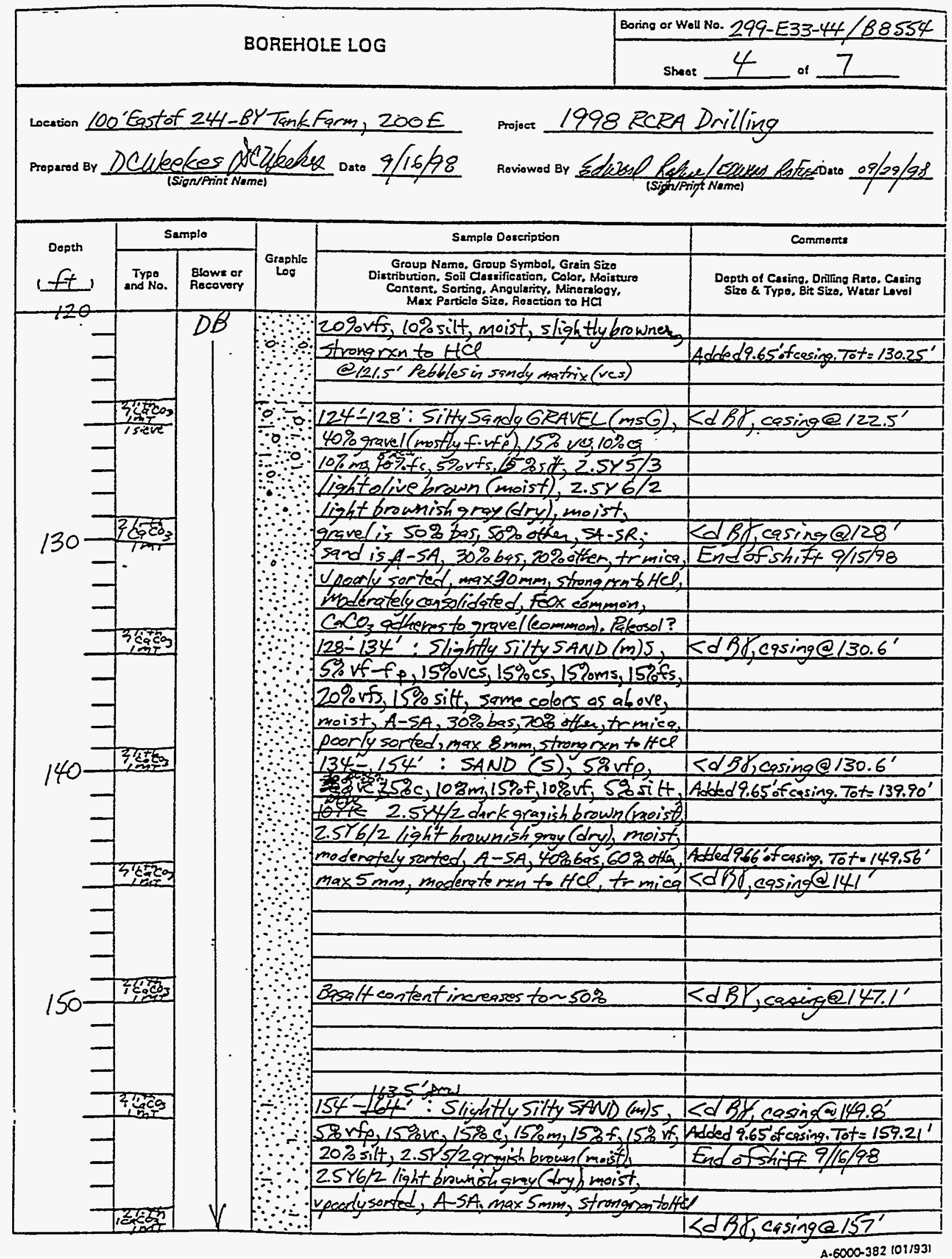




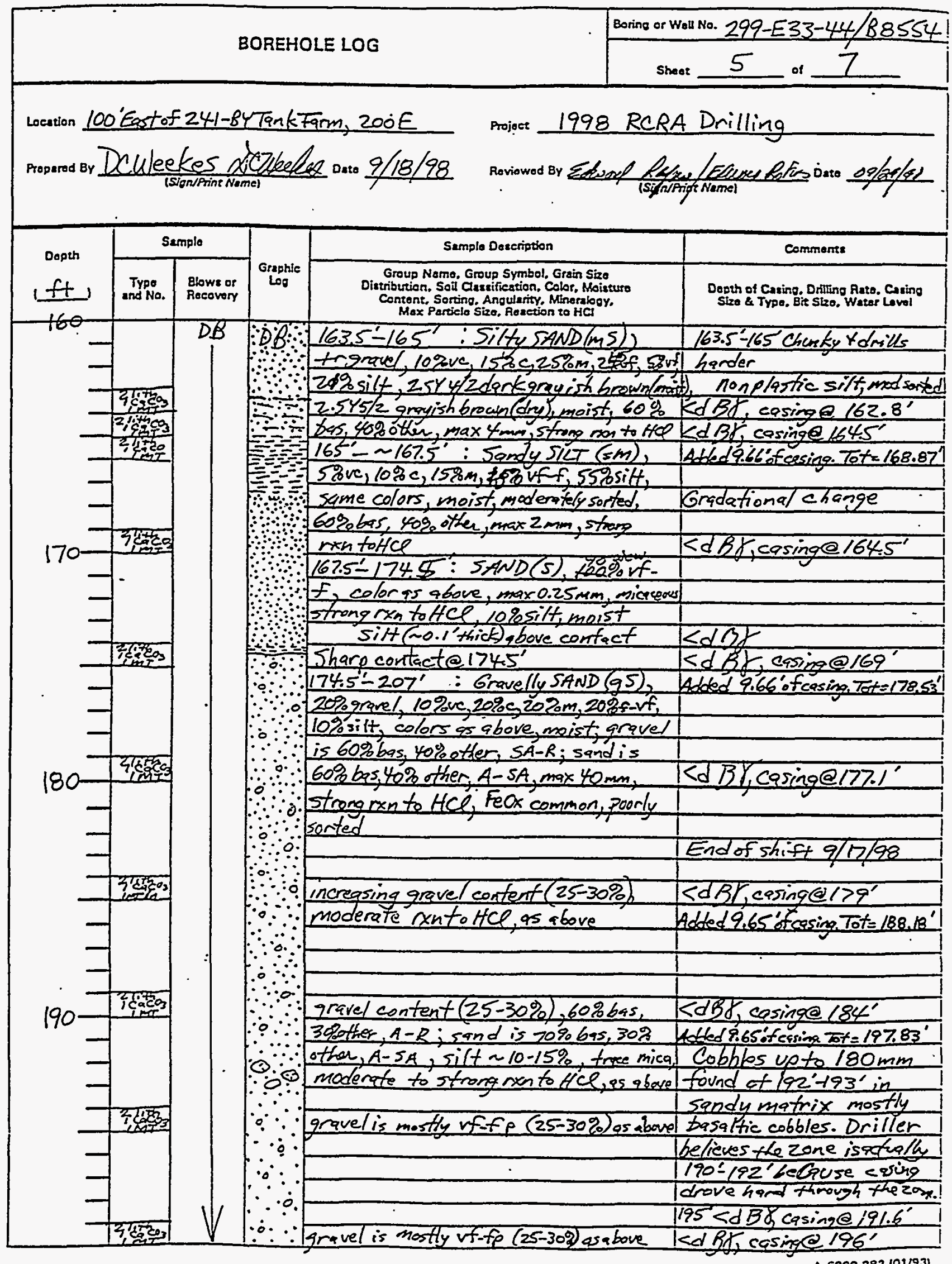




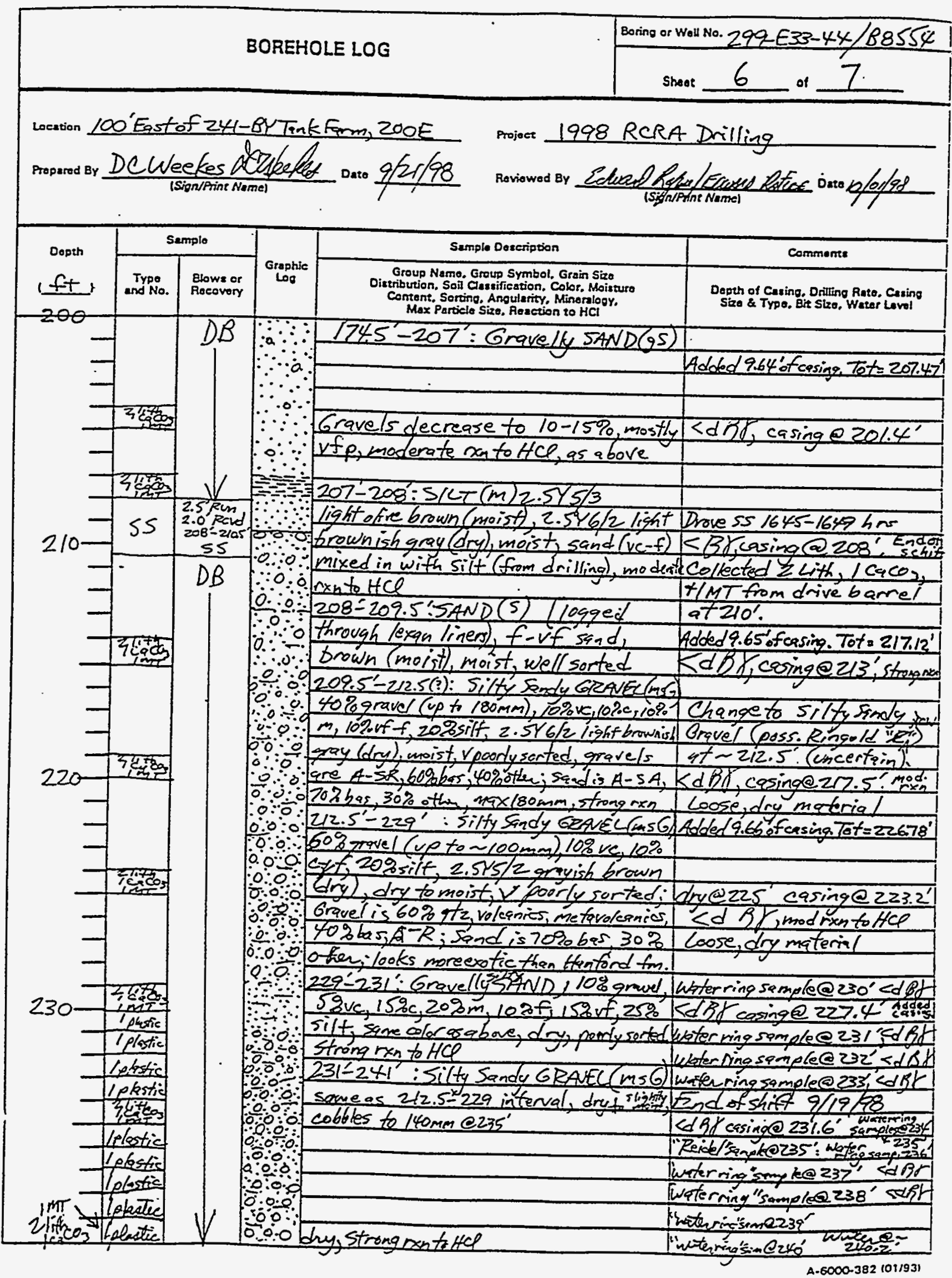




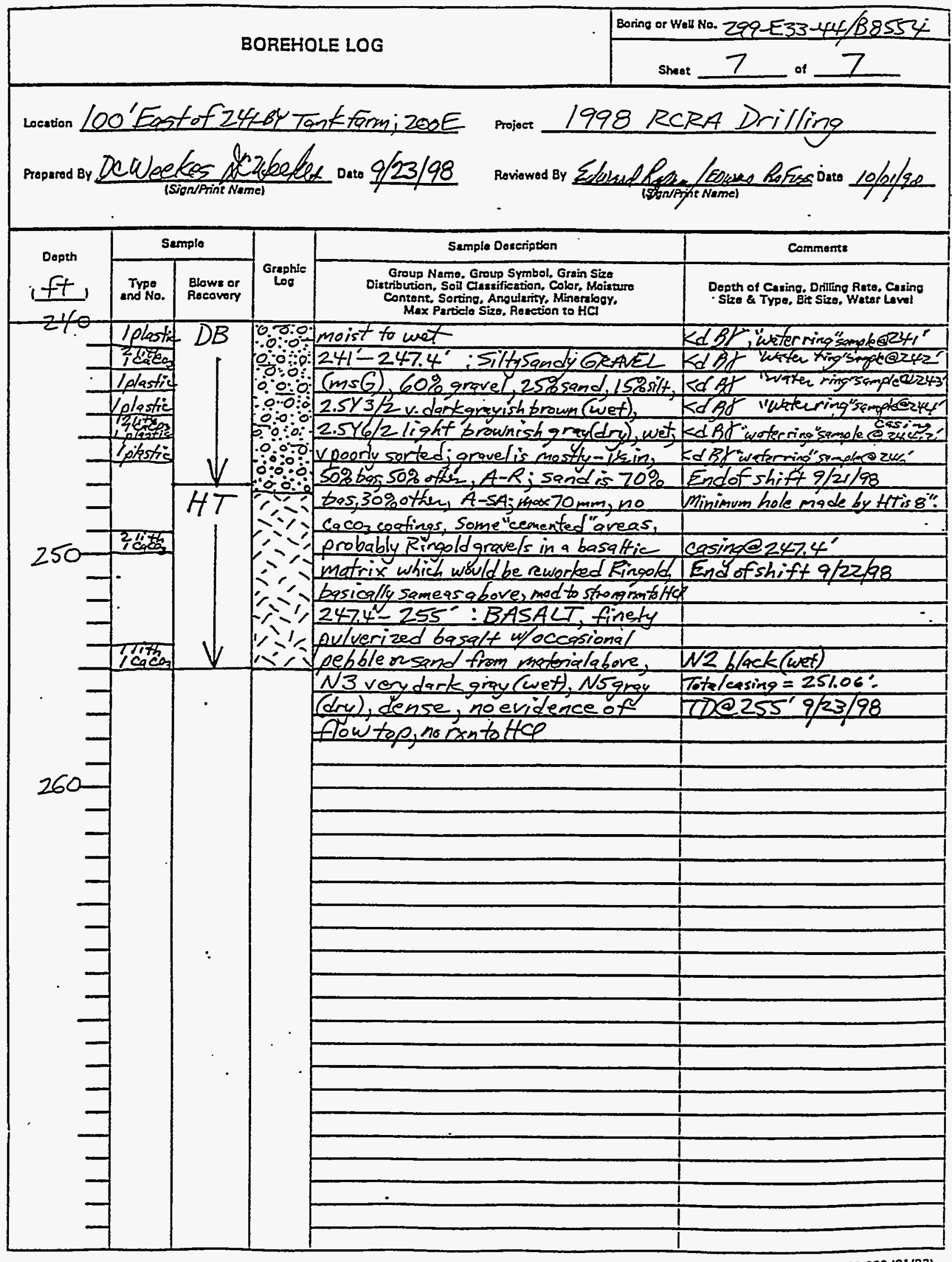

A.6000-382 101/931 
Appendix B

Physical and Chemical Properties Data 


\section{Appendix B}

\section{Physical and Chemical Properties Data}

This Appendix includes the results of laboratory testing for $\mathrm{pH}$, conductivity, particle size distribution, moisture content, total inorganic carbon, and major cations and anions from 1:1 water:sediment extractions.

Moisture content was measured as weight loss after drying an aliquot of the bulk sample at $105^{\circ} \mathrm{C}$ for $24 \mathrm{~h}$ or until weight was constant for two consecutive measurements.

Total inorganic carbon was measured from an air-dried aliquot of the $<2 \mathrm{~mm}$ fraction. The sample was combusted in a total carbon analyzer at $975^{\circ} \mathrm{C}$ and the weight of evolved carbon dioxide was determined and converted to calcium carbonate equivalent. Reagent grade calcium carbonate was used as a standard. Instrument precision was \pm 3 weight percent based on replicate measurements of reagent grade calcium carbonate.

Exchangeable cations were determined by inductively coupled plasma analysis (ICP) after exchange with an ammonium acetate solution. Twenty grams of air dried sediment were mixed with $50 \mathrm{~mL}$ of $1 \mathrm{M}$ ammonium acetate for 16 hours. Electrical conductivity, $\mathrm{pH}$, and major cations and anions were determined from a 1:1 water:sediment extract. The concentrations of major cations were measured with ICP, anions were determined by ion chromatography (IC), and alkalinity was measured by titration. Electrical conductivity and $\mathrm{pH}$ were determined from unfiltered aliquots and cations and anions from aliquots of solution filtered through $0.45 \mu \mathrm{m}$ membranes.

Particle size analysis was done using standard sieve techniques. Samples were oven dried at $105^{\circ} \mathrm{C}$ for $24 \mathrm{~h}$ (or until weight was constant for two consecutive measurements) prior to analysis. 
Table B.1. Moisture Content, pH, and Electrical Conductivity of Samples from Well 299-W33-4

\begin{tabular}{|c|c|c|c|c|}
\hline Depth (ft) & $\begin{array}{l}\text { Moisture } \\
\text { Content } \\
\text { (wt } \%)\end{array}$ & 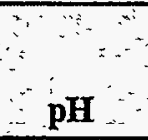 & $\begin{array}{l}\text { Conductivity } \\
\text { (jiS/cm) }\end{array}$ & $\begin{array}{l}\text { Temperature } \\
\left({ }^{\circ} \mathrm{C}\right)\end{array}$ \\
\hline 24 & 5.1 & 8.55 & 163 & 22.3 \\
\hline 25 & 4.9 & 8.55 & 180 & 22.4 \\
\hline 30 & 4.7 & 8.45 & 137 & 22.2 \\
\hline 70 & 7.7 & 8.02 & 323 & 22.3 \\
\hline 75 & 3.0 & 8.55 & 194 & 22.2 \\
\hline 80 & 9.4 & 8.09 & 478 & 22.2 \\
\hline 95 & 4.7 & 8.53 & 242 & 22.2 \\
\hline 100 & 4.3 & 8.44 & 303 & 22.3 \\
\hline 105 & 4.8 & 8.35 & 272 & 22.4 \\
\hline 140 & 3.5 & 8.2 & 292 & 22.2 \\
\hline 150 & 3.5 & 8.23 & 395 & 22.2 \\
\hline 165 & 5.3 & 8.27 & 409 & 22.3 \\
\hline 166 & 14.9 & 7.96 & 913 & 22.5 \\
\hline 170 & 5.9 & 8.07 & 366 & 22.2 \\
\hline 174.5 & & 7.92 & 616 & 22.4 \\
\hline 175 & 5.5 & 8.18 & 374 & 22.2 \\
\hline 205 & 3.5 & 8.3 & 255 & 22.3 \\
\hline 208 & 13.5 & 8.04 & 508 & 22.3 \\
\hline 210 & 2.9 & 8.21 & 375 & 22.3 \\
\hline 230 & 2.6 & 8.42 & 228 & 22.3 \\
\hline 231 & & 8.42 & 182 & 22.3 \\
\hline 232 & & 8.57 & 140 & 22.4 \\
\hline 239 & & 8.52 & 181 & 22.3 \\
\hline 240 & 2.6 & 8.48 & 208 & 22.3 \\
\hline 241 & & 8.57 & 147 & 22.3 \\
\hline 242 & & 8.62 & 120 & 22.3 \\
\hline
\end{tabular}


Table B.2. Particle Size Distribution Data for Samples from Well 299-E33-44

\begin{tabular}{|c|c|c|c|}
\hline $\begin{array}{l}\text { Particle } \\
\text { Size (mm) }\end{array}$ & $\begin{array}{l}\text { Sieve } \\
\text { Sove }\end{array}$ & $\begin{array}{l}\text { Wèight of } \\
\text { Dry } \\
\text { Sample(g) }\end{array}$ & $\begin{array}{l}\text { Weight } \\
\text { Percent }\end{array}$ \\
\hline$\because \because$ & \multicolumn{3}{|c|}{ Depth-70 to:74.5 ft } \\
\hline 2 & 10 & 0.93 & 0.5 \\
\hline 0.88 & 20 & 2.36 & 1.3 \\
\hline 0.425 & 40 & 2.86 & 1.6 \\
\hline 0.25 & 60 & 1.96 & 1.1 \\
\hline 0.106 & 140 & 21.58 & 12.1 \\
\hline 0.075 & 200 & 20.5 & 11.5 \\
\hline \multirow[t]{2}{*}{$<0.075$} & Pan & 127.78 & 71.8 \\
\hline & Total & 177.97 & 100.0 \\
\hline \multicolumn{4}{|c|}{ Depth $230 \mathrm{ft}$} \\
\hline 2 & 10 & 26.9 & 13.7 \\
\hline 0.88 & 20 & 33.5 & 17.1 \\
\hline 0.425 & 40 & 35.59 & 18.1 \\
\hline 0.25 & 60 & 17 & 8.7 \\
\hline 0.106 & 140 & 23.05 & 11.7 \\
\hline 0.075 & 200 & 8 & 4.1 \\
\hline \multirow[t]{2}{*}{$<0.075$} & Pan & 52.35 & 26.7 \\
\hline & Total & 196.39 & 100.0 \\
\hline \multicolumn{4}{|c|}{ Depth 234 ft } \\
\hline 2 & 10 & 408.6 & 51.7 \\
\hline 0.88 & 20 & 69.57 & 8.8 \\
\hline 0.425 & 40 & 65.37 & 8.3 \\
\hline 0.25 & 60 & 37.04 & 4.7 \\
\hline 0.106 & 140 & 51.31 & 6.5 \\
\hline 0.075 & 200 & 15.81 & 2.0 \\
\hline \multirow[t]{2}{*}{$<0.075$} & Pan & 142.88 & 18.1 \\
\hline & Total & 790.58 & 100.0 \\
\hline
\end{tabular}

\begin{tabular}{|c|c|c|c|}
\hline $\begin{array}{l}\text { Particle } \\
\text { Size (mm) }\end{array}$ & $\begin{array}{r}\text { S } \\
\text { Sieve } \\
\end{array}$ & \begin{tabular}{|} 
Weight of \\
Dry Sample \\
(g)
\end{tabular} & $\begin{array}{l}\text { Weight } \\
\text { Percent }\end{array}$ \\
\hline \multicolumn{4}{|c|}{ Depth 125ft } \\
\hline 2 & 10 & 44.03 & 23.7 \\
\hline 0.88 & 20 & 37.64 & 20.2 \\
\hline 0.425 & 40 & 21.83 & 11.7 \\
\hline 0.25 & 60 & 31.49 & 16.9 \\
\hline 0.106 & 140 & 20.22 & 10.9 \\
\hline 0.075 & 200 & 5.59 & 3.0 \\
\hline \multirow[t]{2}{*}{$<0.075$} & Pan & 25.29 & 13.6 \\
\hline & Total & 186.09 & 100.0 \\
\hline \multicolumn{4}{|c|}{ Depth 231 ft } \\
\hline 2 & 10 & 37 & 18.9 \\
\hline 0.88 & 20 & 41.63 & 21.3 \\
\hline 0.425 & 40 & 40.35 & 20.6 \\
\hline 0.25 & 60 & 17.11 & 8.8 \\
\hline 0.106 & 140 & 17.75 & 9.1 \\
\hline 0.075 & 200 & 5.99 & 3.1 \\
\hline \multirow[t]{2}{*}{$<0.075$} & Pan & 35.57 & 18.2 \\
\hline & Total & 195.4 & 100.0 \\
\hline \multicolumn{4}{|c|}{ Depth $236 \mathrm{ft}$} \\
\hline 2 & 10 & 184.6 & 41.7 \\
\hline 0.88 & 20 & 56.3 & 12.7 \\
\hline 0.425 & 40 & 51.42 & 11.6 \\
\hline 0.25 & 60 & 23.73 & 5.4 \\
\hline 0.106 & 140 & 32.73 & 7.4 \\
\hline 0.075 & 200 & 10.89 & 2.5 \\
\hline \multirow[t]{2}{*}{$<0.075$} & Pan & 82.95 & 18.7 \\
\hline & Total & 442.62 & 100.0 \\
\hline
\end{tabular}


Table B.2. (contd)

\begin{tabular}{|c|c|c|c|}
\hline $\begin{array}{c}\text { Particle } \\
\text { Size (mm) }\end{array}$ & Sieve & $\begin{array}{l}\text { Weight of } \\
\text { Dample (g) }\end{array}$ & $\begin{array}{l}\text { Weight } \\
\text { Percent }\end{array}$ \\
\hline. & \multicolumn{2}{|c|}{ Depth 241 ft } & $\therefore$ \\
\hline 2 & 10 & 659.8 & 67.1 \\
\hline 0.88 & 20 & 85.3 & 8.7 \\
\hline 0.425 & 40 & 65.92 & 6.7 \\
\hline 0.25 & 60 & 31.44 & 3.2 \\
\hline 0.106 & 140 & 41.34 & 4.2 \\
\hline 0.075 & 200 & 12.3 & 1.3 \\
\hline \multirow[t]{2}{*}{$<0.075$} & Pan & 86.75 & 8.8 \\
\hline & Total & 982.85 & 100.0 \\
\hline
\end{tabular}

\begin{tabular}{|c|c|c|c|}
\hline $\begin{array}{l}\text { Particle } \\
\text { Size (imm) }\end{array}$ & $\begin{array}{l}\text { Sieve } \\
\text { Sen }\end{array}$ & $\begin{array}{l}\text { Weíght'of } \\
\text { Dry Sample } \\
\text { (g) }\end{array}$ & $\begin{array}{l}\text { Weight } \\
\text { Percent }\end{array}$ \\
\hline \multicolumn{4}{|c|}{ Depth243 ff 2} \\
\hline 2 & 10 & 367.7 & 74.3 \\
\hline 0.88 & 20 & 40.17 & 8.1 \\
\hline 0.425 & 40 & 27.31 & 5.5 \\
\hline 0.25 & 60 & 13.88 & 2.8 \\
\hline 0.106 & 140 & 16.15 & 3.3 \\
\hline 0.075 & 200 & 5.4 & 1.1 \\
\hline \multirow[t]{2}{*}{$<0.075$} & Pan & 24 & 4.9 \\
\hline & Total & 494.61 & 100.0 \\
\hline
\end{tabular}

\begin{tabular}{|c|c|c|c|}
\hline \multirow{4}{*}{$\begin{array}{c}\text { Particle } \\
\text { Size (mm) }\end{array}$} & Sieve & $\begin{array}{r}\text { Weight of } \\
\text { Dry } \\
\text { Sample (g) }\end{array}$ & $\begin{array}{c}\text { Weight } \\
\text { Percent }\end{array}$ \\
\hline \multicolumn{4}{|c|}{ Depth 245.ft } \\
\hline 2 & 10 & 326.2 & 59.8 \\
0.88 & 20 & 80.8 & 14.8 \\
0.425 & 40 & 47.17 & 8.6 \\
0.25 & 60 & 21.42 & 3.9 \\
0.106 & 140 & 22.92 & 4.2 \\
0.075 & 200 & 6.75 & 1.2 \\
$<0.075$ & Pan & 40.23 & 7.4 \\
\cline { 2 - 4 } & Total & $\mathbf{5 4 5 . 4 9}$ & $\mathbf{1 0 0 . 0}$ \\
\hline
\end{tabular}


Table B.3. Major Cation and Anion Concentrations from 1:1 Water:Sediment Extracts of Samples from Well 299-E33-44

\begin{tabular}{|c|c|c|c|c|c|c|c|c|c|}
\hline \multirow[b]{2}{*}{$\begin{array}{l}\text { Depth } \\
\text { (ft) }\end{array}$} & \multicolumn{6}{|c|}{ Cations $(\overline{\mathrm{m}} \mathrm{g} / \mathrm{L})$} & \multirow{2}{*}{$\begin{array}{l}\text { Total } \\
\text { Cations } \\
\text { (meqL) }\end{array}$} & \multirow{2}{*}{$\begin{array}{c}\text { Monoyalent } \\
\text { Cations } \\
(\%)\end{array}$} & \multirow{2}{*}{$\begin{array}{l}\text { Divalent } \\
\text { Cations } \\
\text { (\%) }\end{array}$} \\
\hline & $\begin{array}{c} \\
\therefore \mathbf{B a}\end{array}$ & $\mathrm{Ca}$ & $\mathbf{K}$ & $\mathbf{M g}$ & $\mathbf{N a}$ & $\begin{array}{r}\mathbf{S} \mathbf{r} \\
-\mathbf{r}\end{array}$ & & & \\
\hline 24 & 0.00 & 7.58 & 0.00 & 2.03 & 23.61 & 0.05 & 1.57 & 65.27 & 34.73 \\
\hline 25 & 0.05 & 9.27 & 1.44 & 2.22 & 21.31 & 0.05 & 1.61 & 59.83 & 40.17 \\
\hline 30 & 0.06 & 8.75 & 3.12 & 2.67 & 10.65 & 0.04 & 1.20 & 45.21 & 54.79 \\
\hline 70 & 0.05 & 23.12 & 0.00 & 6.75 & 15.96 & 0.13 & 2.41 & 28.85 & 71.15 \\
\hline 75 & 0.03 & 9.74 & 0.00 & 3.00 & 21.25 & 0.04 & 1.66 & 55.73 & 44.27 \\
\hline 80 & 0.06 & 27.81 & 2.80 & 9.25 & 41.09 & 0.15 & 4.01 & 46.34 & 53.66 \\
\hline 95 & 0.02 & 12.39 & 0.00 & 3.63 & 26.28 & 0.06 & 2.06 & 55.44 & 44.56 \\
\hline 100 & 0.00 & 14.90 & 0.98 & 4.17 & 30.60 & 0.08 & 2.44 & 55.48 & 44.52 \\
\hline 105 & 0.06 & 15.31 & 1.47 & 4.76 & 22.34 & 0.08 & 2.17 & 46.56 & 53.44 \\
\hline 140 & 0.02 & 23.43 & 3.56 & 6.75 & 10.94 & 0.12 & 2.29 & 24.70 & 75.30 \\
\hline 150 & 0.00 & 31.67 & 6.59 & 9.15 & 15.52 & 0.18 & 3.18 & 26.53 & 73.47 \\
\hline 165 & 0.02 & 27.06 & 12.58 & 8.05 & 36.62 & 0.17 & 3.93 & 48.70 & 51.30 \\
\hline 166 & 0.06 & 76.87 & 14.66 & 23.89 & 58.59 & 0.43 & 8.73 & 33.47 & 66.53 \\
\hline 170 & 0.07 & 33.27 & 9.16 & 8.59 & 26.61 & 0.17 & 3.76 & 36.98 & 63.02 \\
\hline 174.5 & 0.08 & 47.08 & 15.94 & 16.85 & 39.64 & 0.28 & 5.87 & 36.29 & 63.71 \\
\hline 175 & 0.05 & 31.79 & 13.95 & 10.34 & 27.39 & 0.18 & 3.99 & 38.80 & 61.20 \\
\hline 205 & 0.04 & 17.75 & 14.14 & 5.16 & 24.93 & 0.11 & 2.76 & 52.40 & 47.60 \\
\hline 208 & 0.01 & 42.65 & 13.18 & 11.58 & 33.36 & 0.23 & 4.87 & 36.68 & 63.32 \\
\hline 210 & 0.08 & 24.65 & 18.84 & 6.69 & 35.17 & 0.15 & 3.80 & 52.98 & 47.02 \\
\hline 230 & 0.00 & 15.46 & 10.33 & 3.94 & 22.94 & 0.09 & 2.36 & 53.49 & 46.51 \\
\hline 231 & 0.08 & 17.79 & 10.12 & 4.21 & 21.32 & 0.11 & 2.42 & 48.94 & 51.06 \\
\hline 232 & 0.00 & 10.43 & 7.52 & 2.45 & 18.56 & 0.07 & 1.72 & 58.00 & 42.00 \\
\hline 239 & 0.07 & 12.75 & 2.94 & 3.11 & 12.12 & 0.09 & 1.50 & 40.22 & 59.78 \\
\hline 240 & 0.04 & 14.79 & 2.46 & 4.12 & 15.17 & 0.10 & 1.80 & 40.10 & 59.90 \\
\hline 241 & 0.02 & 9.71 & 0.00 & 2.46 & 10.72 & 0.06 & 1.15 & 40.40 & 59.60 \\
\hline 242 & 0.00 & 9.51 & 0.00 & 2.50 & 6.97 & 0.06 & 0.99 & 30.78 & 69.22 \\
\hline
\end{tabular}


Table B.3. (contd)

\begin{tabular}{|c|c|c|c|c|c|c|c|}
\hline \multirow{2}{*}{ Depth (ft) } & \multicolumn{5}{|c|}{ Anions (mg/L) } & \multirow{2}{*}{$\begin{array}{l}\text { Total } \\
\text { Anions } \\
\text { (meq/L) }\end{array}$} & \multirow{2}{*}{ Electrical Balance (\%) } \\
\hline & $\begin{array}{l}\text { Alkalinity } \\
\text { as } \mathbf{C a C O}\end{array}$ & $\mathbf{F}$ & $\mathbf{C l}^{-}$ & $\mathrm{NO}_{3}$ & $\begin{array}{l}\mathbf{S O}_{4} \\
\mathbf{S}\end{array}$ & & \\
\hline 24 & 54.98 & 0.39 & 1.31 & 10.53 & 9.17 & 1.52 & 3.59 \\
\hline 25 & 61.65 & 0.48 & 2.14 & 7.20 & 13.45 & 1.72 & -6.28 \\
\hline 30 & 43.73 & 0.27 & 1.23 & 9.72 & 8.21 & 1.25 & -4.10 \\
\hline 70 & 48.76 & 0.40 & 8.80 & 14.16 & 69.00 & 2.91 & -18.90 \\
\hline 75 & 34.16 & 0.45 & 2.78 & 12.85 & 33.93 & 1.70 & -2.41 \\
\hline 80 & 47.49 & 0.50 & 13.89 & 66.76 & 104.48 & 4.62 & -14.08 \\
\hline 95 & 75.73 & 0.34 & 5.28 & 25.31 & 39.89 & 2.92 & -34.47 \\
\hline 100 & 133.40 & 0.38 & 6.60 & 41.31 & 57.69 & 4.74 & -63.94 \\
\hline 105 & 56.04 & 0.21 & 6.06 & 33.81 & 49.64 & 2.88 & -28.26 \\
\hline 140 & 63.64 & 0.35 & 10.28 & 37.94 & 54.09 & 3.32 & -36.51 \\
\hline 150 & 329.02 & 0.32 & 14.21 & 48.31 & 65.15 & 9.13 & -96.69 \\
\hline 165 & 36.36 & 0.55 & 26.92 & 64.96 & 81.33 & 4.26 & -7.94 \\
\hline 166 & 44.02 & 0.58 & 58.62 & 77.90 & 242.85 & 8.88 & -1.61 \\
\hline 170 & 38.81 & 0.43 & 6.90 & 15.78 & 118.69 & 3.72 & 1.17 \\
\hline 174.5 & 46.55 & 0.21 & 52.02 & 57.37 & 120.86 & 5.85 & 0.41 \\
\hline 175 & 64.10 & 0.34 & 18.86 & 17.15 & 95.15 & 4.09 & -2.48 \\
\hline 205 & 44.67 & 0.48 & 12.68 & 7.38 & 47.60 & 2.39 & 14.50 \\
\hline 208 & 40.17 & 0.62 & 31.40 & 27.78 & 128.69 & 4.85 & 0.54 \\
\hline 210 & 56.51 & 0.62 & 8.94 & $<0.06$ & 110.53 & 3.72 & 2.11 \\
\hline 230 & 47.18 & 0.70 & 2.22 & 4.85 & 52.15 & 2.13 & 10.23 \\
\hline 231 & 32.79 & 0.34 & 1.35 & 2.19 & 26.16 & 1.29 & 60.93 \\
\hline 232 & 34.23 & 0.38 & 1.47 & 2.23 & 27.82 & 1.36 & 23.50 \\
\hline 239 & 44.17 & 0.65 & 2.43 & $<0.06$ & 38.45 & 1.79 & -17.64 \\
\hline 240 & 46.47 & 0.69 & 2.66 & $<0.06$ & 40.98 & & \\
\hline 241 & 36.69 & 0.56 & 1.46 & 0.53 & 24.73 & 1.33 & -14.00 \\
\hline 242 & 27.97 & 0.38 & 1.24 & 2.39 & 15.08 & 0.97 & 1.87 \\
\hline
\end{tabular}


Table B.4. Cation Exchange Capacity and $\mathrm{CaCO}_{3}$ Content of Sediment Samples from Well 299-E33-44

\begin{tabular}{|c|c|c|c|}
\hline Depth (ft) & $\begin{array}{l}\text { Total CEC } \\
\text { (meq/100 g) }\end{array}$ & $\begin{array}{l}\text { Divalent } \\
\text { Cations } \\
(\%)\end{array}$ & $\begin{array}{l}\mathrm{CaCO} \text { Content } \\
(w t \%)\end{array}$ \\
\hline 5 & 9.25 & 88.38 & 10.80 \\
\hline 24 & 6.38 & 88.81 & 0.97 \\
\hline 25 & 5.87 & 91.47 & 1.10 \\
\hline 30 & 5.28 & 93.92 & 1.01 \\
\hline 65 & 5.02 & 94.06 & 1.18 \\
\hline 70 & 6.74 & 93.79 & 2.00 \\
\hline 75 & 5.21 & 92.77 & 2.68 \\
\hline 80 & 6.07 & 91.28 & 1.22 \\
\hline 95 & 4.73 & 92.86 & .2 .95 \\
\hline 100 & 5.37 & 92.40 & 1.86 \\
\hline 105 & 5.03 & 92.81 & 1.56 \\
\hline 140 & 4.55 & 94.99 & 2.44 \\
\hline 150 & 4.71 & 93.04 & 1.03 \\
\hline 165 & 5.71 & 90.51 & 1.55 \\
\hline 166 & 7.11 & 92.41 & 2.28 \\
\hline 170 & 5.47 & 95.85 & 1.70 \\
\hline 174.5 & & & 2.34 \\
\hline 175 & 6.09 & 93.68 & 1.83 \\
\hline 200 & 5.71 & 92.91 & 0.73 \\
\hline 205 & 5.18 & 92.33 & 0.92 \\
\hline 208 & 8.64 & 94.52 & 2.20 \\
\hline 210 & 6.21 & 90.87 & 1.00 \\
\hline 230 & 6.15 & 93.13 & 0.89 \\
\hline 231 & & & 1.43 \\
\hline 232 & & & 0.72 \\
\hline 239 & . & & 0.95 \\
\hline 240 & 6.58 & 91.93 & 1.19 \\
\hline 241 & & & 0.68 \\
\hline 242 & & & 0.56 \\
\hline
\end{tabular}


Appendix C

Geophysical Logs 


\section{Appendix C}

\section{Geophysical Logs.}

This appendix contains the high purity, germanium spectral gamma-ray log; the sodium iodide spectral gamma-ray log (with units pCi/g); the sodium iodide spectral gamma-ray log (with units counts/ second); and the neutron moisture log. All logs were run by Waste Management Federal Services, Inc., Northwest and log data analyses completed by Three Rivers Scientific Company. Included with each log are a Log Header sheet, Acceptance QA Processing data, and a Log Analysis Summary Report. In addition, a description of the method used to convert the $\mathrm{pCi} / \mathrm{g}$ values from the sodium iodide log to counts per second values is included in this appendix. 


\section{RLS Spectral Gamma-Ray Borehole Survey \\ Waste Management Federal Services NW}

\section{Log Header}

Project: RCRA Drilling -1998

Well: $299-$ E33 -44

Log Type: HPGe Spectral Gamma-Ray

Borehole Information

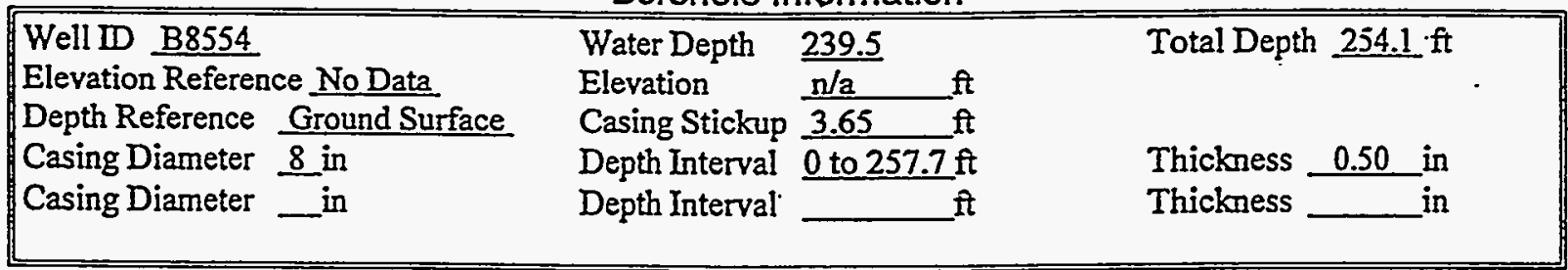

Logging Information

\begin{tabular}{|ll|}
\hline Log Type: & HPGe Spectral Gamma Ray . \\
Company & Waste Management Federal Services NW \\
Date/Archive File Name & Sept 24, 1998 H2E33044 \\
Logging Engineers & A.Pearson \\
Instrument Series & RLSG3.1 \\
Logging Unit & RLS2 \\
Depth Interval & 0 to $180 \mathrm{ft} \quad$ Prefix B217 \\
& 177 to 253.5 ft Prefix B218 \\
& 253 to 225 ft Prefix B218 (repeat) \\
& Sep 11, 1998 \\
Instrument Calibration Date & WHC-SD-EN-TI-292, Rev. 0 \\
\hline
\end{tabular}

Analysis Information

$\begin{array}{ll}\text { Company } & \text { Three Rivers Scientific } \\ \text { Analyst } & \text { Randall Price } \\ \text { Date } & \text { - Sept 30, 1998 }\end{array}$

Notes Cs-137 was identified near the surface $(0.5$ to $3.0 \mathrm{ft})$ with a maximum concentration of $3 \mathrm{pCi} / \mathrm{g}$ and at . intermittent locations (less than $0.3 \mathrm{pCi} / \mathrm{g}$ ) to the bottom of the well. No other man made radionuclides were detected. 


\section{RLS Spectral Gamma Ray Borehole Survey Waste Management Federal Services NW}

Project: . RCRA Drilling-1998 . Log Date: . Sept. 24; 1998 Borehole: 299-E33-44 (B8554) Naturally Occurring Radionuclides

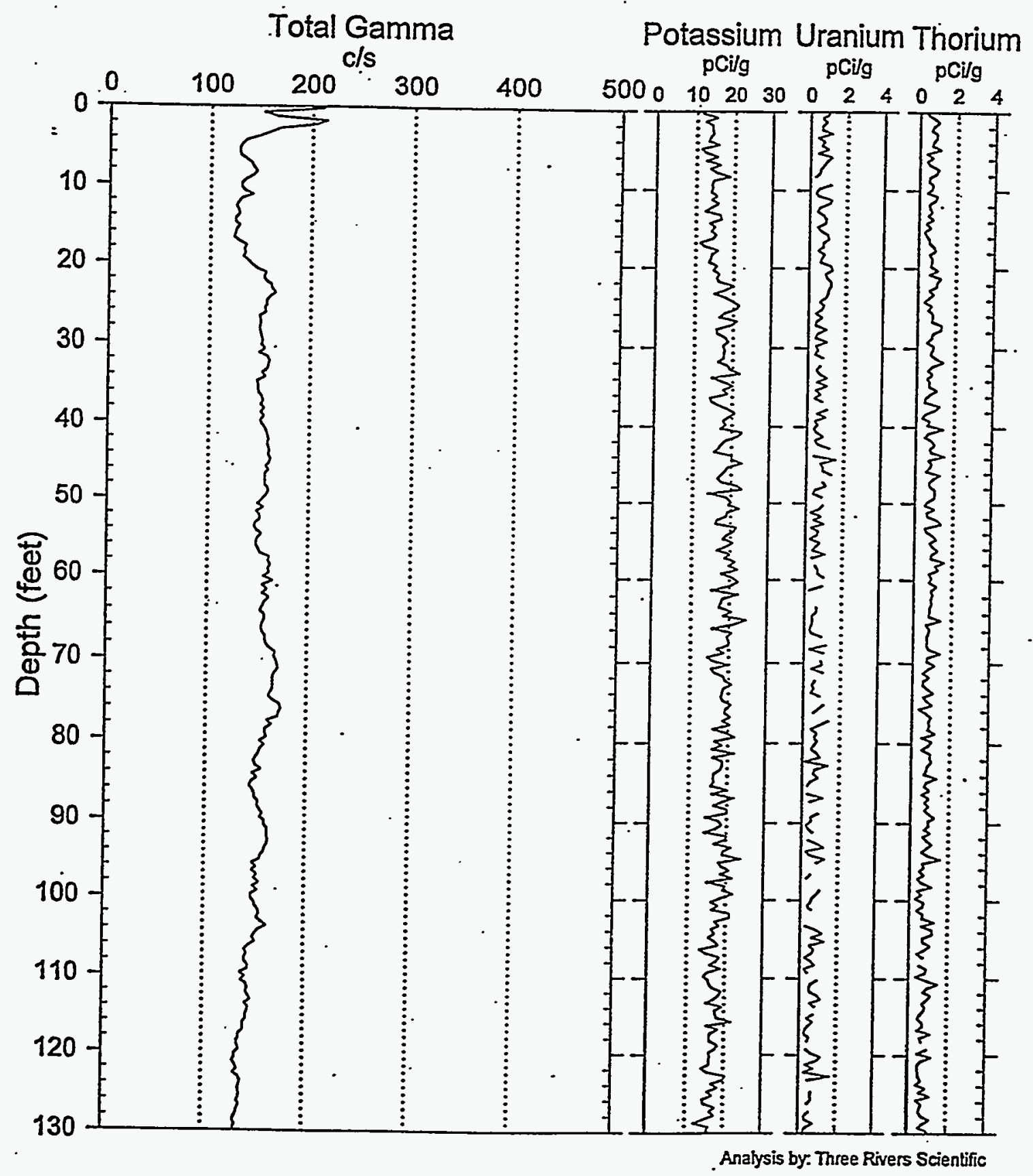




\section{RLS Spectral Gamma Ray Borehole Survey Waste Management Federal Services.NW}

Project: $\quad$ RCRA Drilling - 1998 . Log Date: Sept. 24, 1998. Borehole: 299-E33-44 (B8554) Naturally Occurring Radionuclides

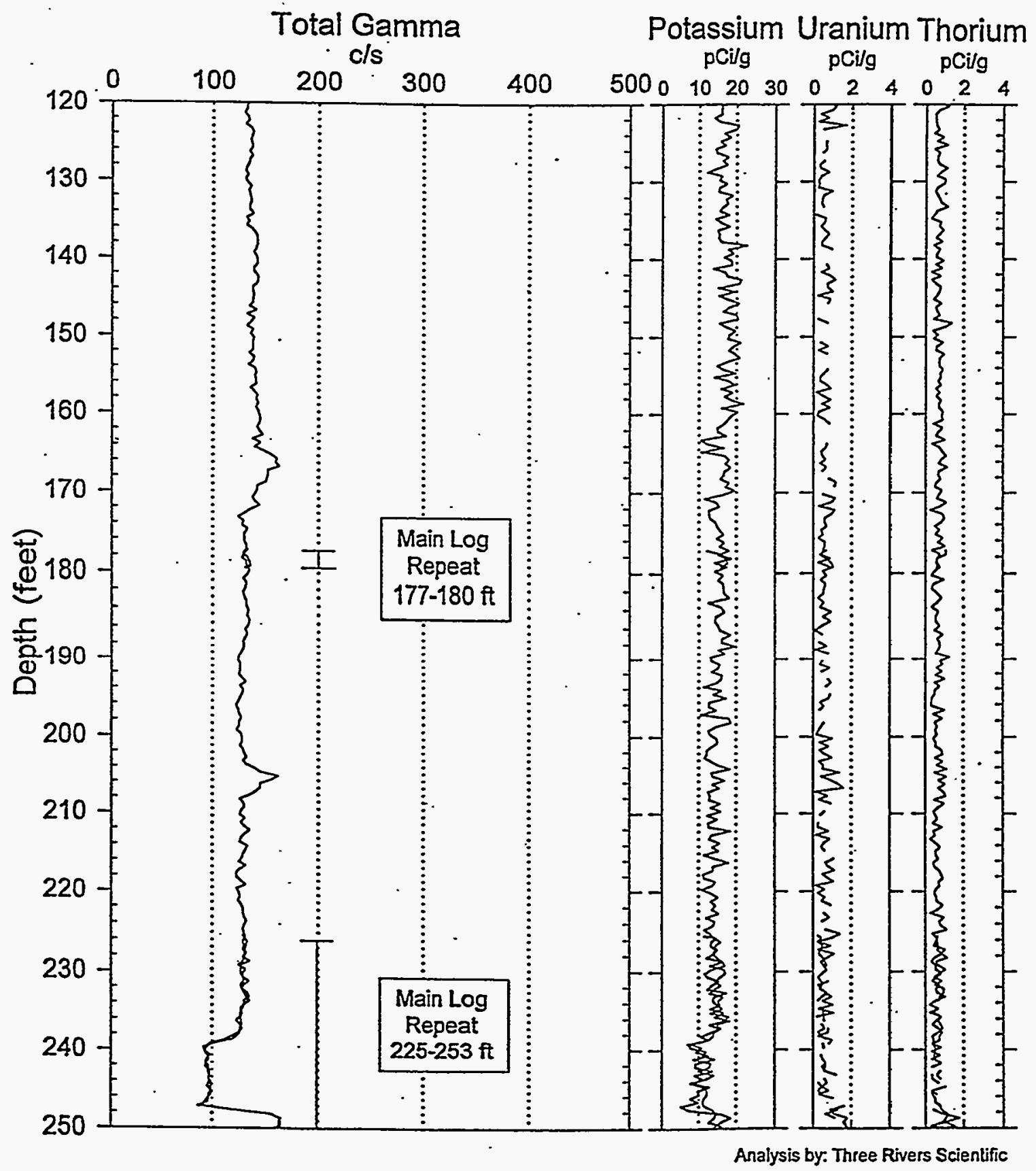




\section{RLS Spectral Gamma Ray Borehole Survey Waste Management Federal Services.NW}

Project: : RCRA Drilling-1998 Log Date: . . Sept. 24, 1998. Borehole: 299-E33-44 (B8554) Naturally Occurring Radionuclides

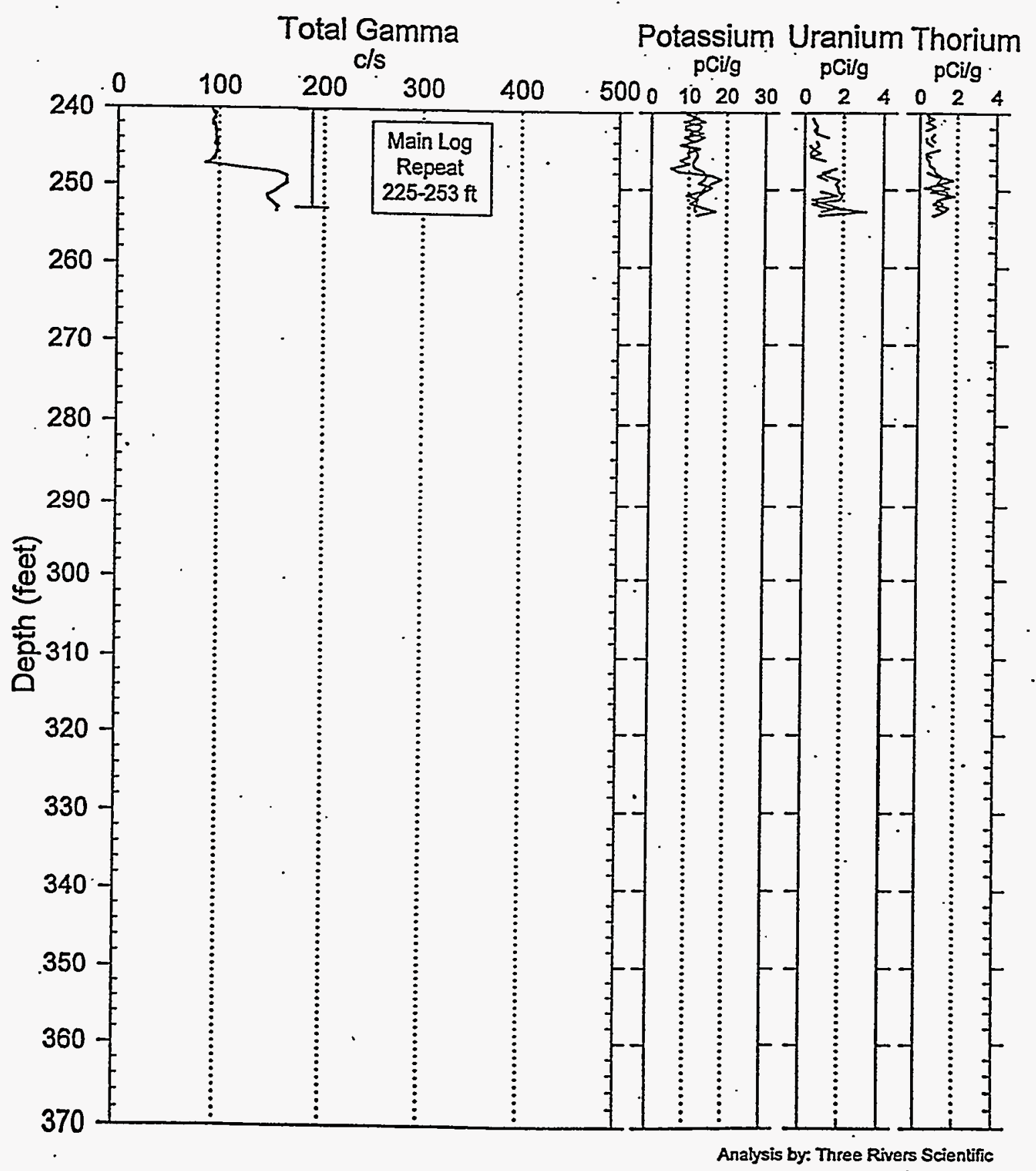




\section{RLS Spectral Gamma-Ray Borehole Survey Waste Management Federal Services NW}

Project: RCRA Drilling - 1998

Log Date: Sept. 24, 1998

Borehole: 299-E33-44 (B8554) Man-Made Radio-lsotopes of Concern

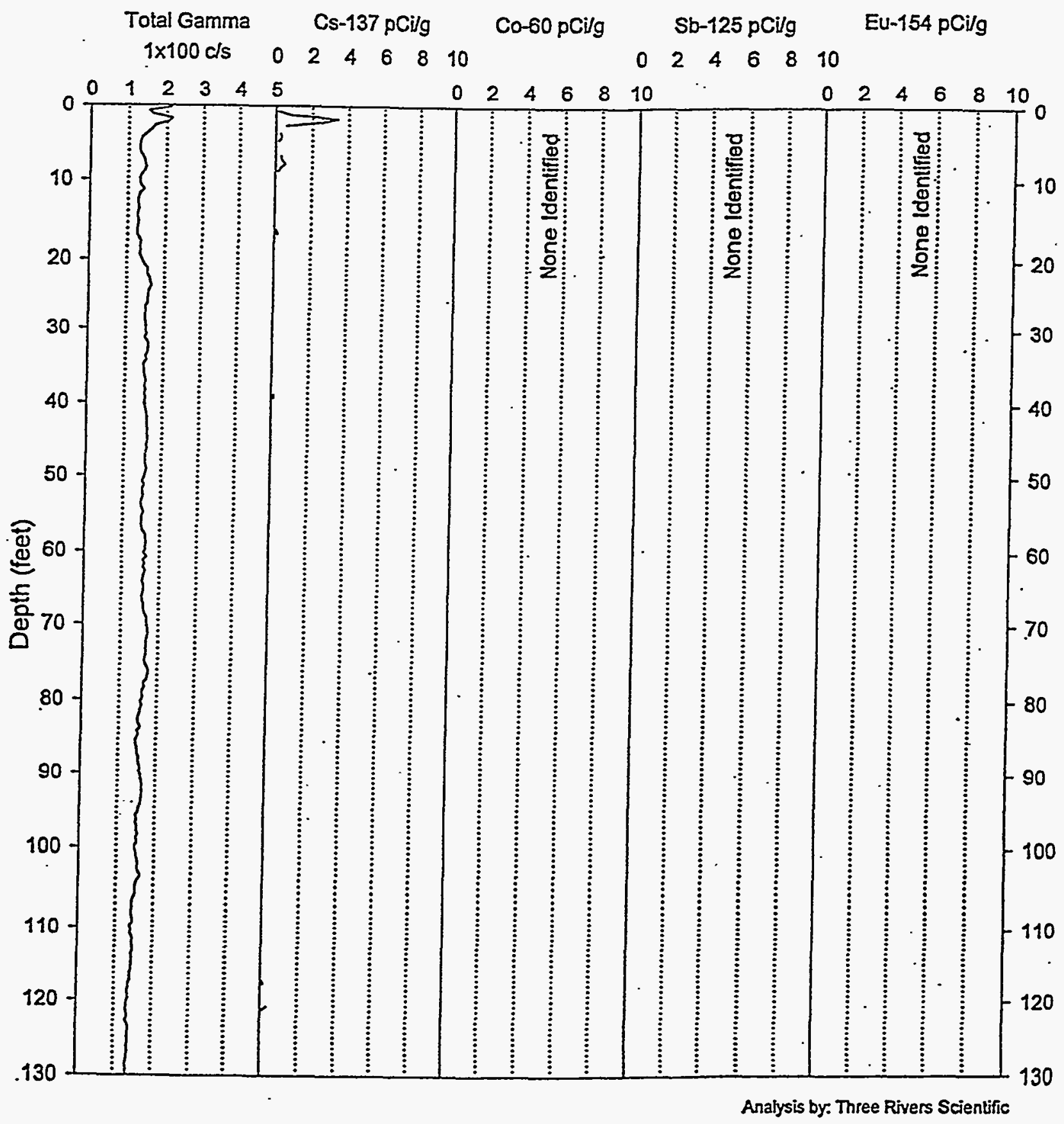




\section{RLS Spectral Gamma-Ray Borehole Survey}

Waste Management Federal Services NW

Troject: RCRA Drilling - 1998 Log Date: Sept. 24, 1998 Borehole: 299-E33-44 (B8554) Man-Made Radio-Isotopes of Concern

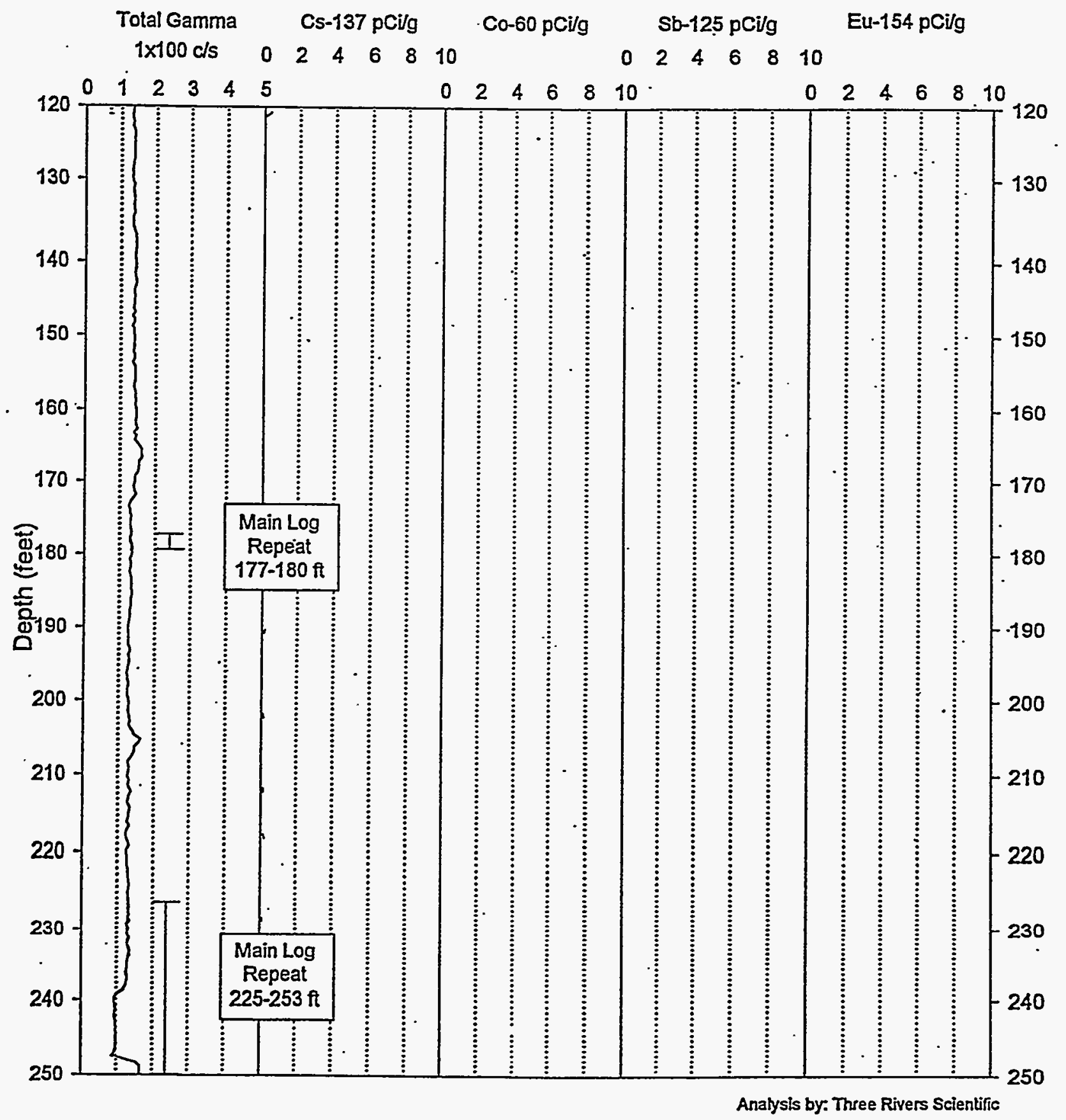




\section{RLS Spectral Gamma-Ray Borehole Survey Waste Management Federal Services NW}

Project: RĊRA Drilling - $1998 \quad \therefore$ Log Date: Sept. 24, 1998 Borehole: 299-E33-44 (B8554) Man-Made Radio-Isotopes of Concern

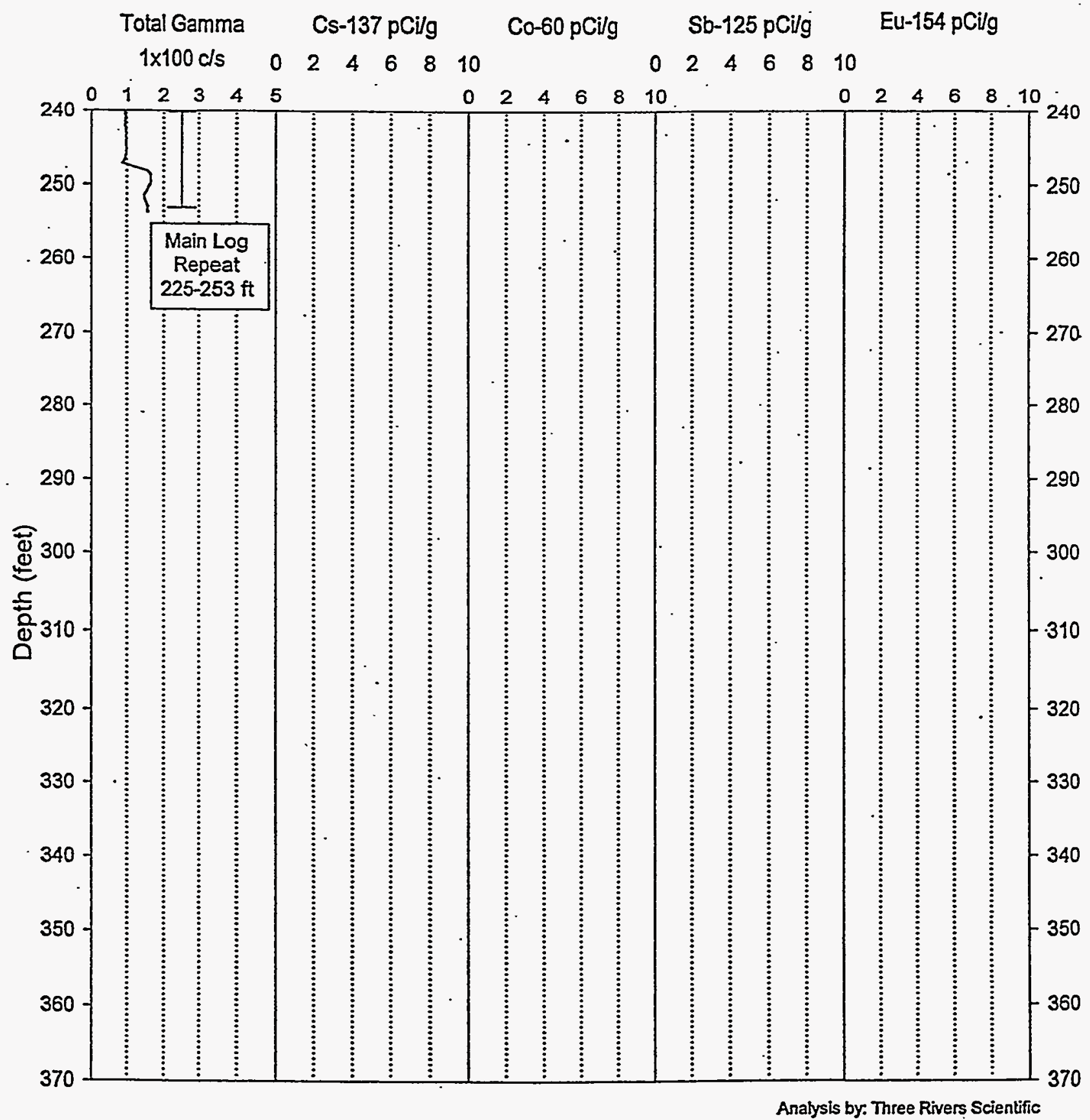




\section{RLS Spectral Gamma Ray Borehole Survey Waste Management Federal Services NW}

Project: RCRA Drilling - 1998 Log Date: . Sept. 24, 1998 Borehole: $299-E 33-44$ (B8554) $\therefore$ Natural Radionuclides \& Cs-137

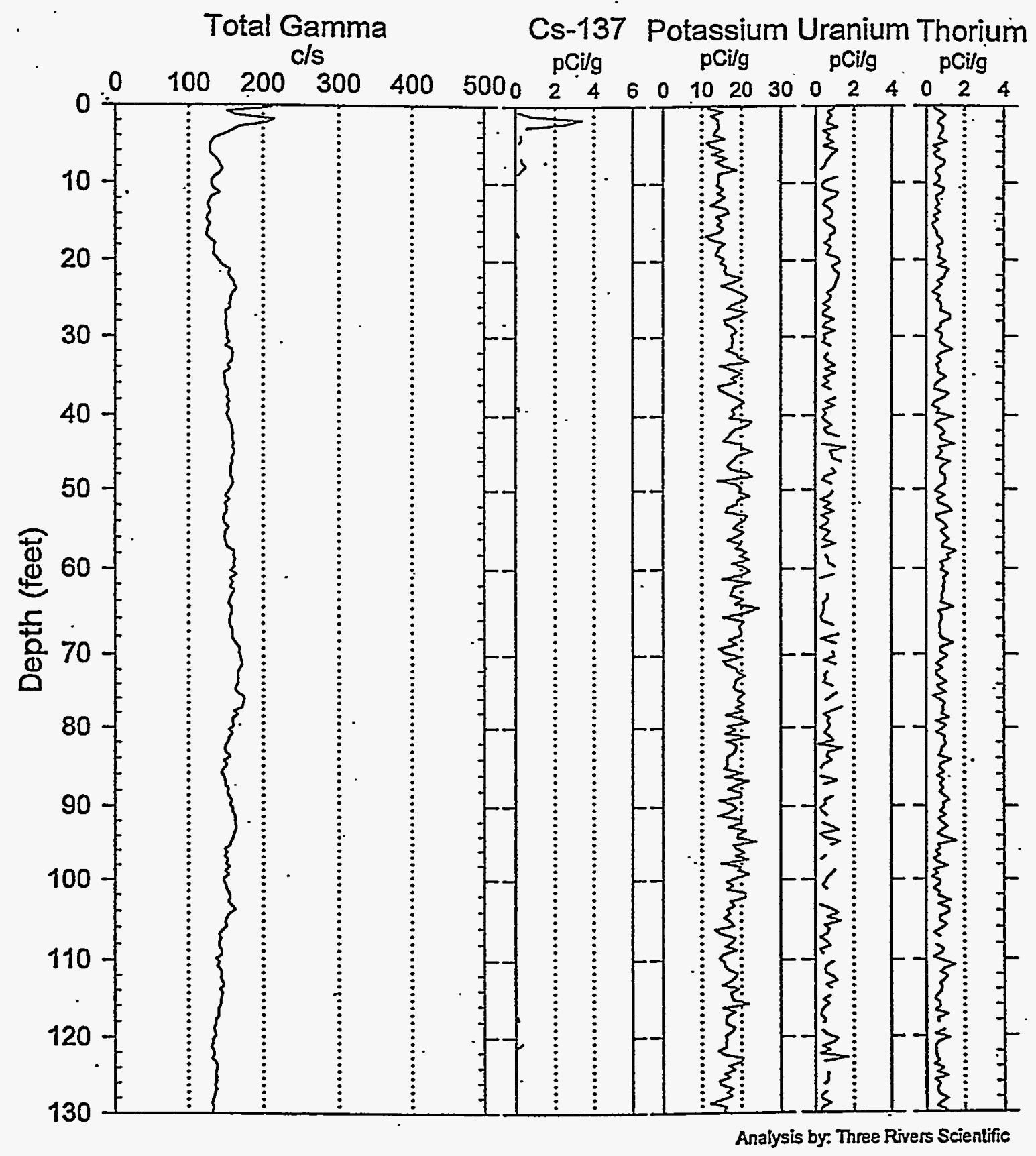




\section{RLS Spectral Gamma Ray Borehole Survey Waste Management Federal Services NW}

Project: - RCRA Drilling - 1998. - Log Date: . Sept. 24, 1998 Borehole: 299-E33-44 (B8554) Natural Radionuclides \& Cs-137.

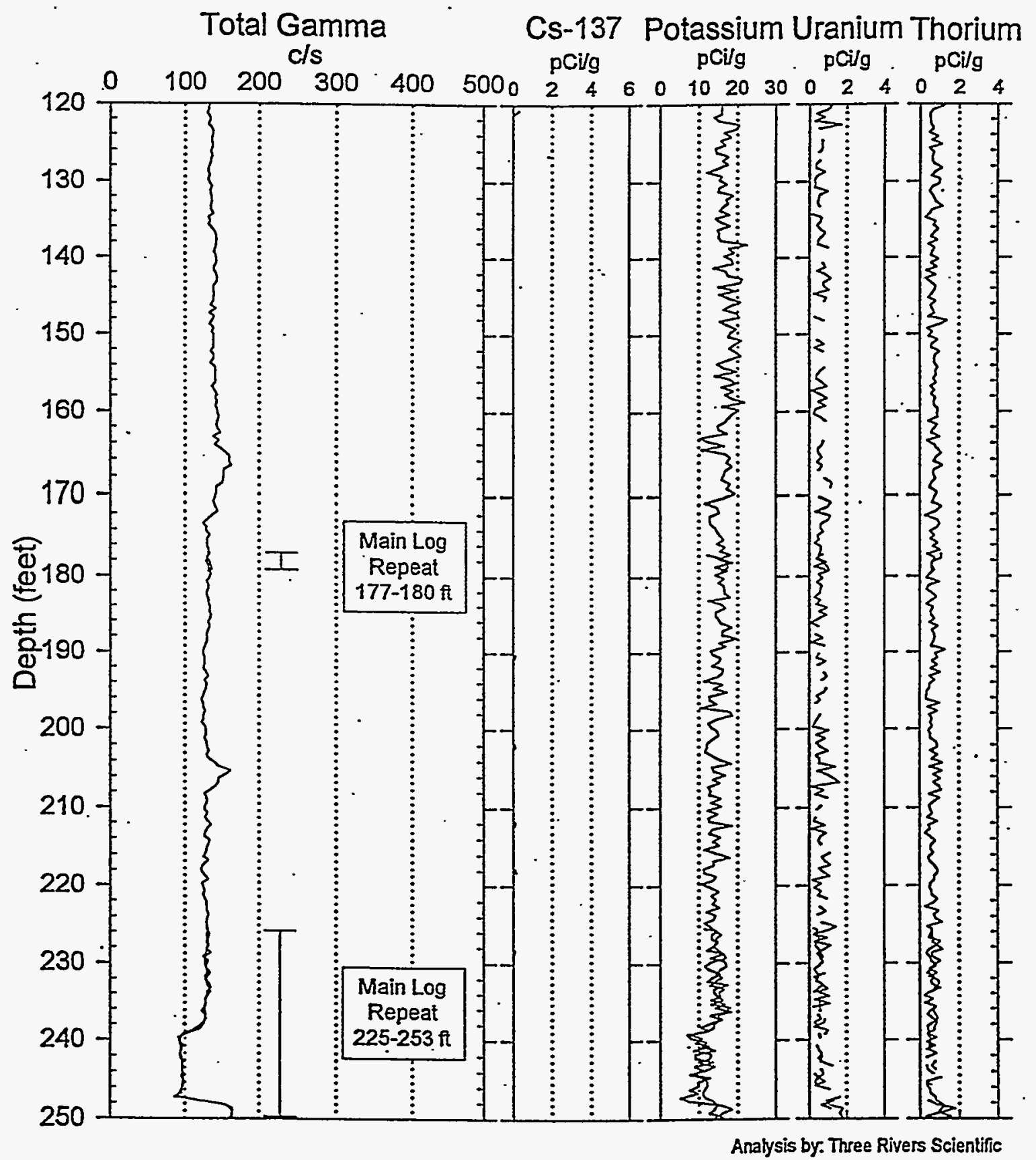




\section{RLS Spectral Gamma Ray Borehole Survey Waste Management Federal Services NW}

Project: RCRA Drilling - 1998 Log Date:. . Sept. 24, 1998 Borehole: 299-E33-44 (B8554) Natural Radionuclides \& Cs-137

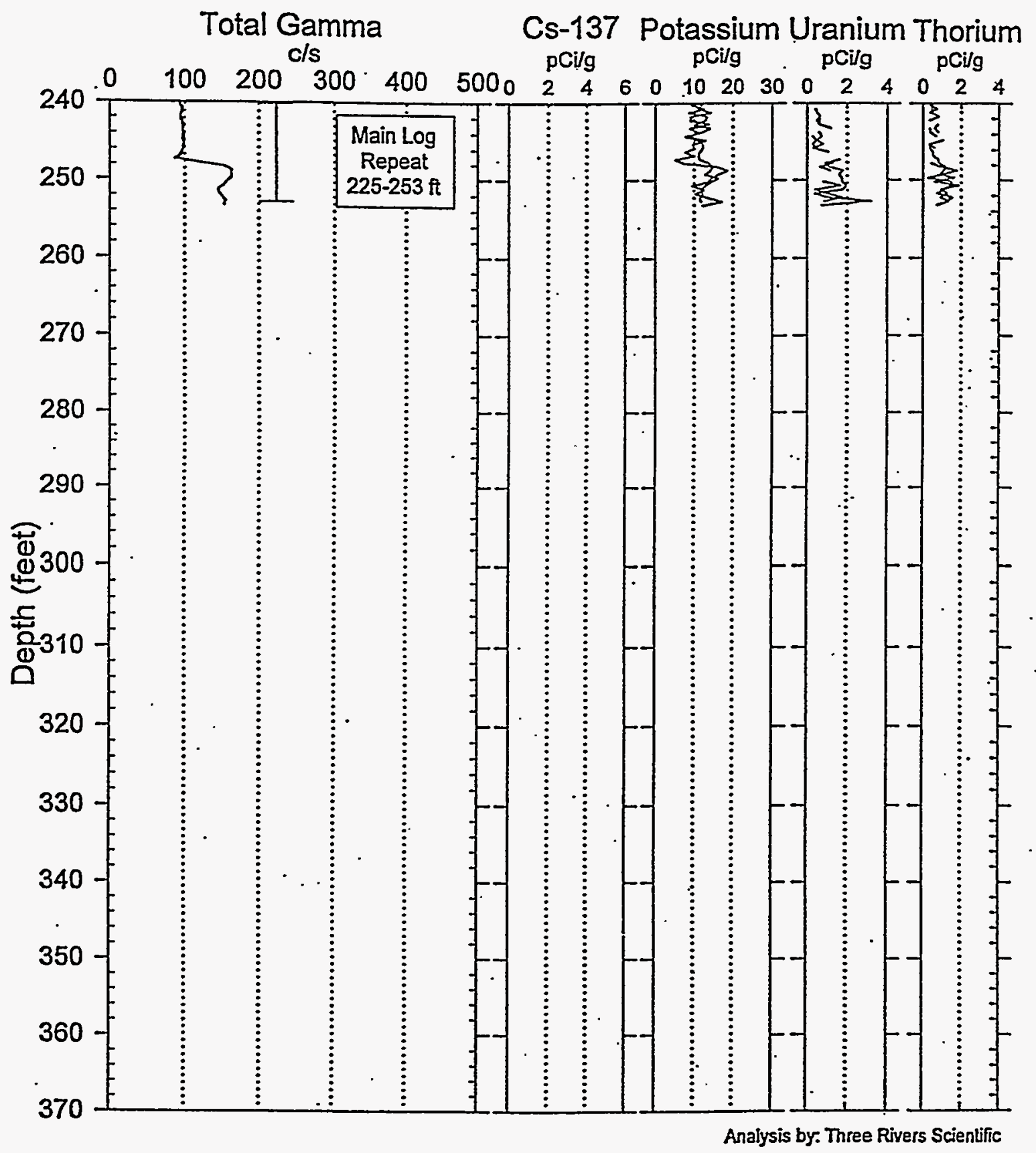




\section{RLS Spectral Gamma-Ray Borehole Survey Acceptance QA Processing}

Project: RCRA Drilling - 1998 Log. Date: Sept 24, 1998

Borehole: 299-E33-44 (B8554) Compare Main Log \& Repeat

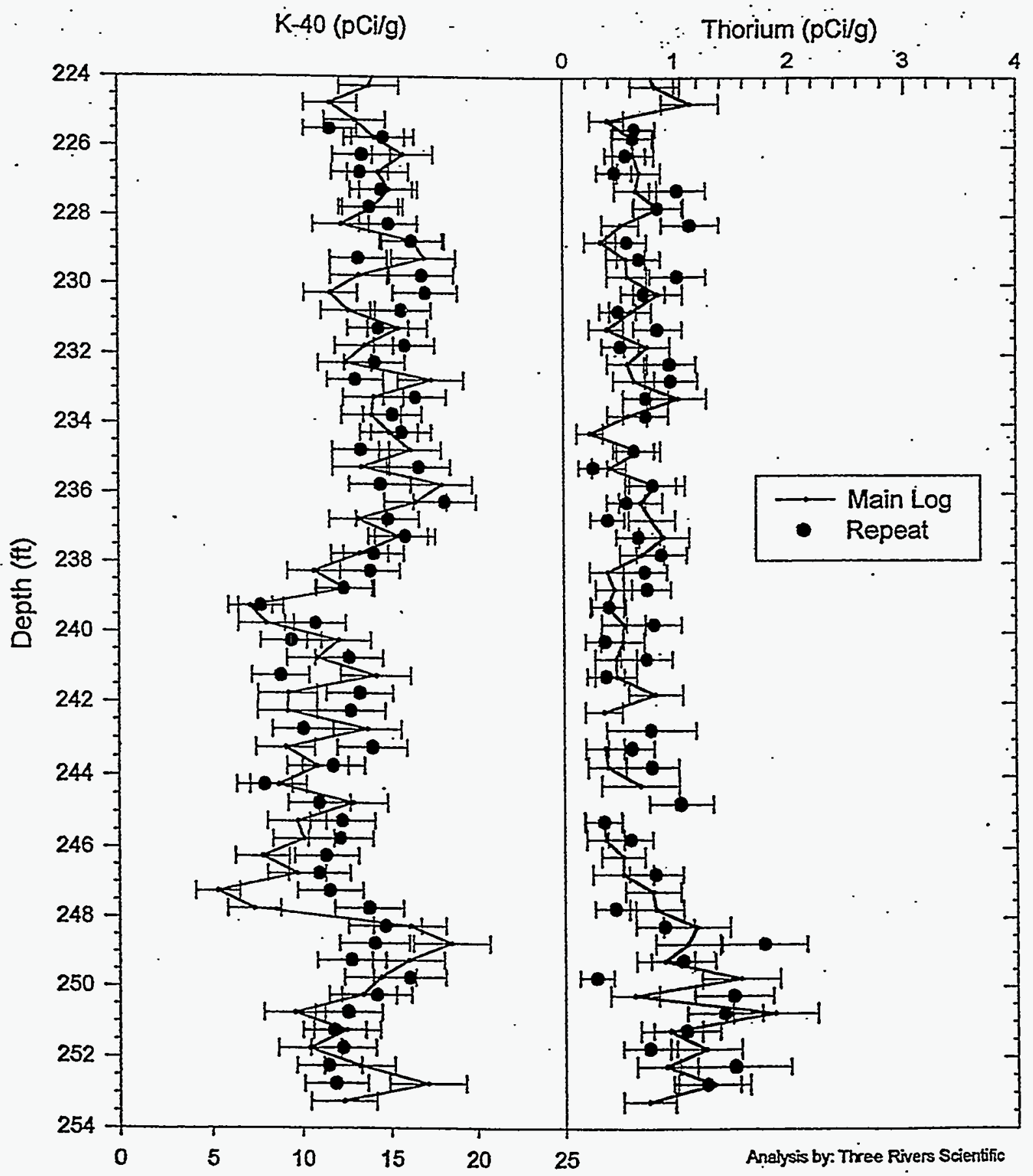

C.12 


\section{RLS Spectral Gamma-Ray Borehole Survey \\ Waste Management Federal Services NW}

$\therefore \quad$ Log Analysis Summary Report

Project: $\quad$ RCRA Drilling - 1998

Well ID: $\quad$ 299-E33-44

Log Type: HPGe Spectral Gamma-Ray ～· Log Dates: Sept 24, 1998

\section{General Notes:}

Total gamma is a response to man made gamma-ray emitters and formation lithology from surface to 4 feet, and a function of formation lithology below 4 feet.

The system dead-time limit was not exceeded (less than 5\%) for the borehole survey.

System Performance Verify: The pre- and post-log verification was performed using coleman $\$ 2$ mantle, and passed the equipment performance check. The maximum FWHM for the $583 \mathrm{keV}$ gamma ray photo peak for both survey dates was $2.20 \mathrm{keV}$. The maximum acceptable FWHM resolution is $3.10 \mathrm{keV}$ for probe RLSG3.1 on the log dates.

Repeat Interval: The repeat interval, 225 to $253 \mathrm{fh}$ agrees with the main log within acceptable limits, refer to the Acceptance QA Processing plot.

Environmental Corrections: The KUT and man made radionuclide concentrations have been corrected for casing attenuation (entire well) and water inside the casing (below $239.5 \mathrm{ft}$ ) for a centralized detector configuration. No casing correction was applied to the total gamma due to Compton downscatter interference.

\section{Radionuclides:}

Cs-137 was identified near the surface $\left(0.5\right.$ to $\left.3.0^{\circ} \mathrm{ft}\right)$ with a maximum concentration of $3 \mathrm{pCi} / \mathrm{g}$ at 2 feet and at intermittent locations (less than $0.3 \mathrm{pCi} / \mathrm{g}$ at depths greater than $10 \mathrm{ft}$ ) to the bottom of the well. No other man made radionuclides detected. 


\section{RLS Spectral Gamma Ray Borehole Survey \\ Waste Management Federal Services NW}

\section{Log Header}

Project: RCRA Drilling Well: 299-E33-44

\section{Log Type: $\quad$ Nal Spectral Gamma Ray}

Borehole Information

\begin{tabular}{|c|c|c|c|c|c|}
\hline Well ID & B8554 & Water Depth & 239.5 & Total Depth & $254.1 \mathrm{ft}$ \\
\hline $\begin{array}{l}\text { Elevanon Reterenc } \\
\text { Depth Reference }\end{array}$ & $\frac{\text { UN }}{\text { Ground Level }}$ & Elevation & UN & & \\
\hline Casing Diameter & 8 in $\mathrm{WD}$ & Depth Interval & 0 to $254.1 \mathrm{ft}$ & Thi & 0.5 \\
\hline Casing Diameter & in ID & Depth Interval & ft & Thickness & in \\
\hline
\end{tabular}

Logging Information

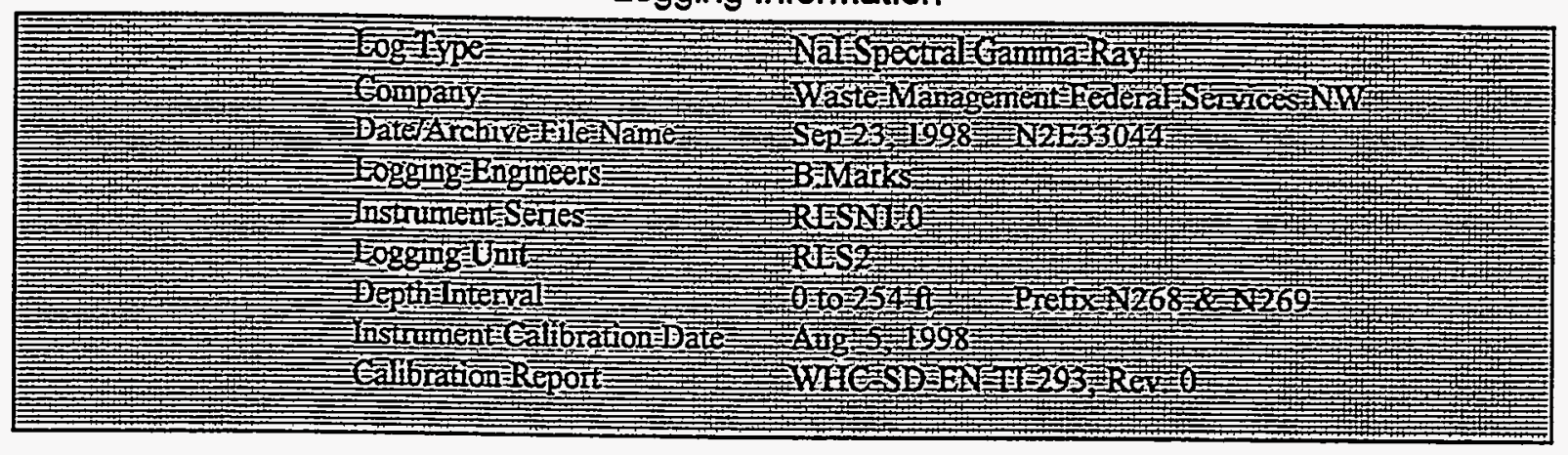

Analysis Information

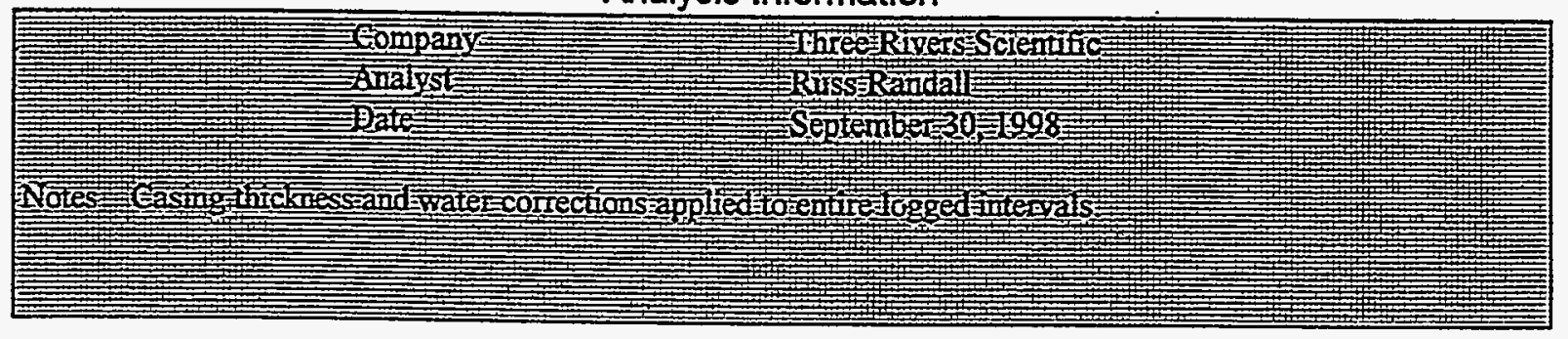


RLS Nal KUT Processed Data.

Waste Management Federal Services NW.

Project: RCRA Drilling

Log Date : Sep 23, 1998

Borehole: 299-E33-44

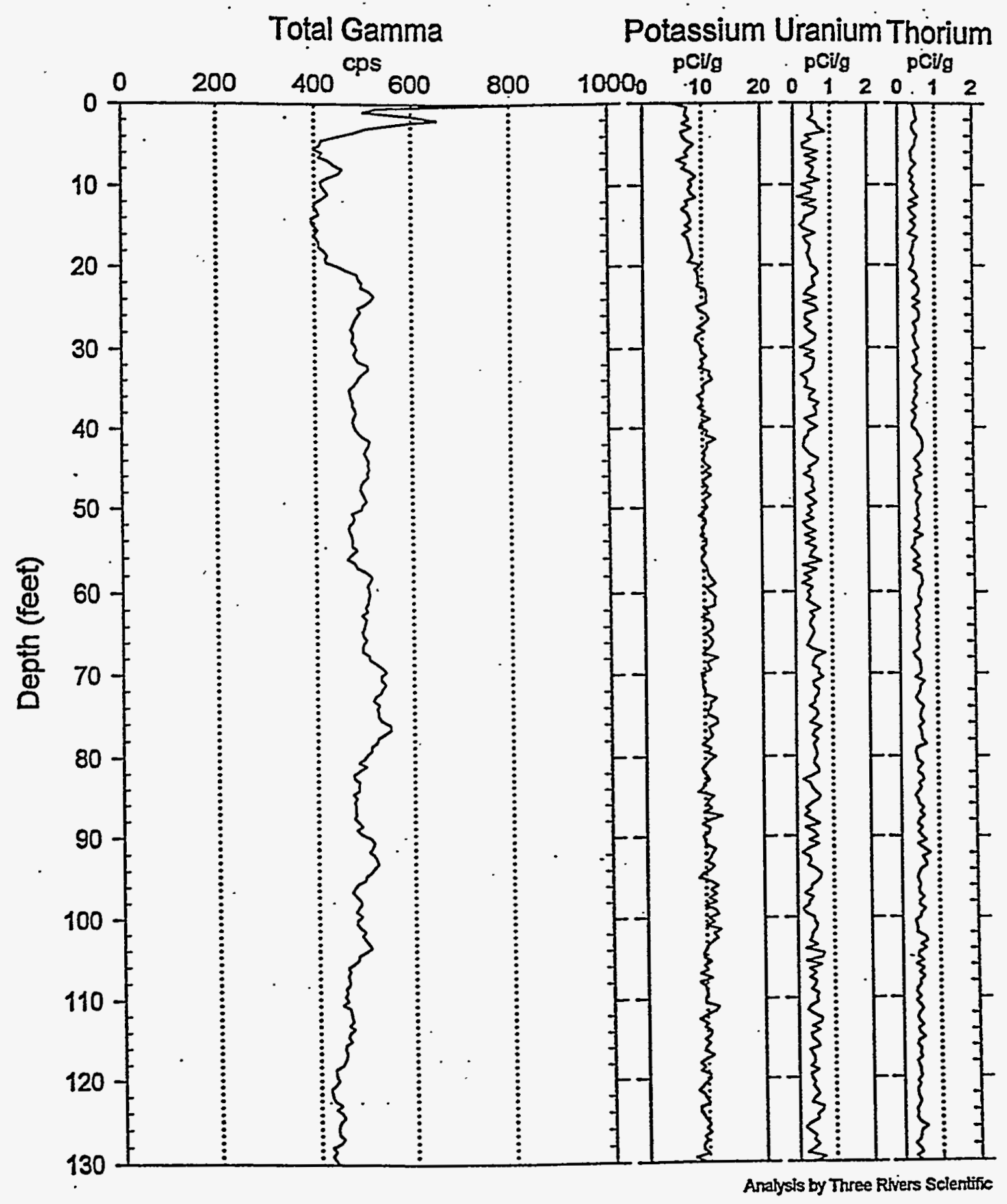




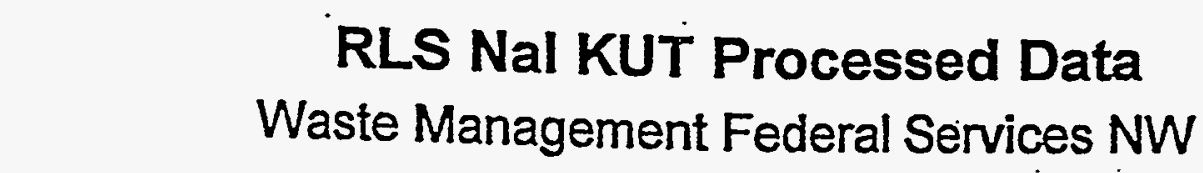

Project: RCRA Drilling

Borehole: 299-E33-44

Log Date : Sep 23, 1998

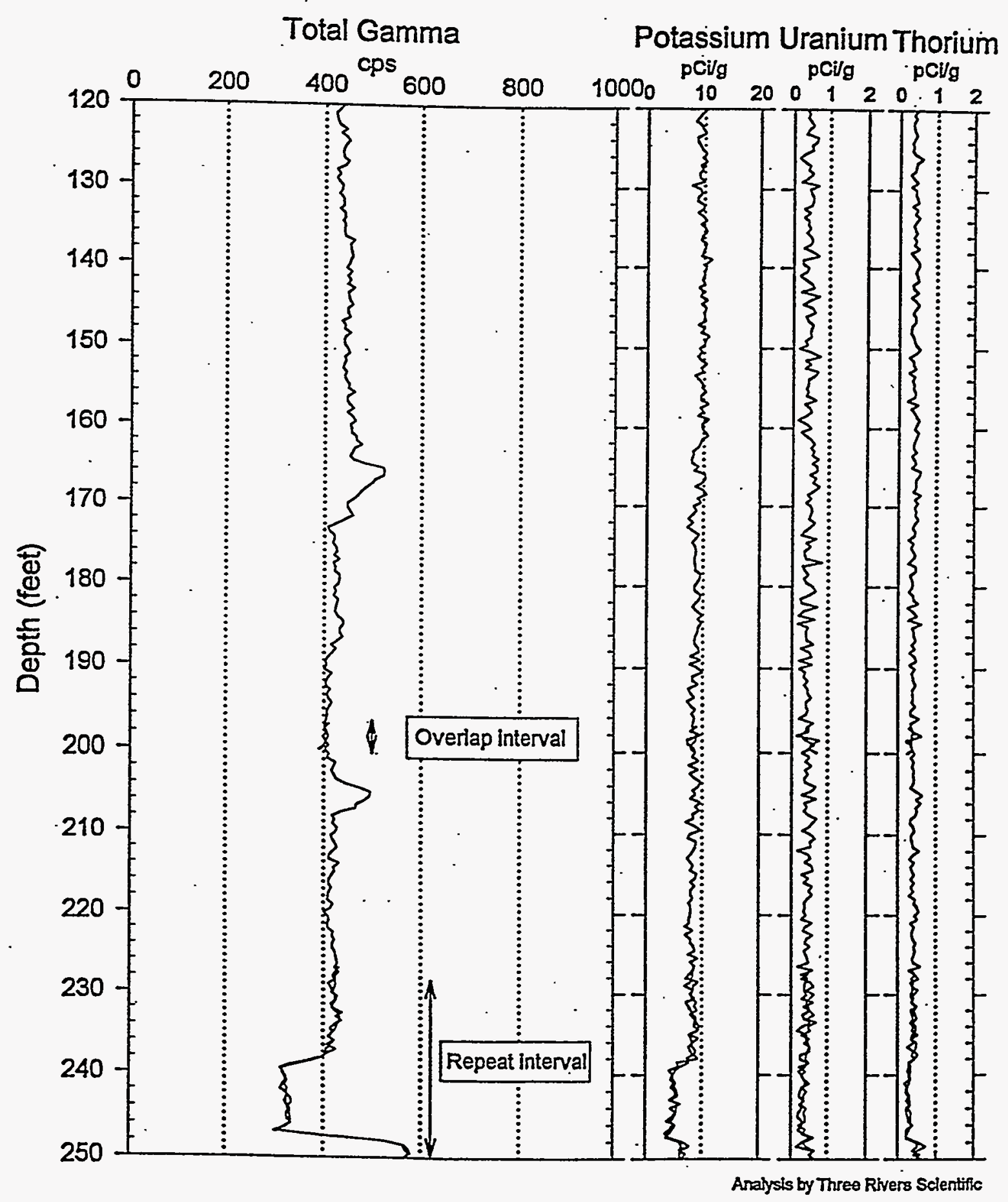

C.16 


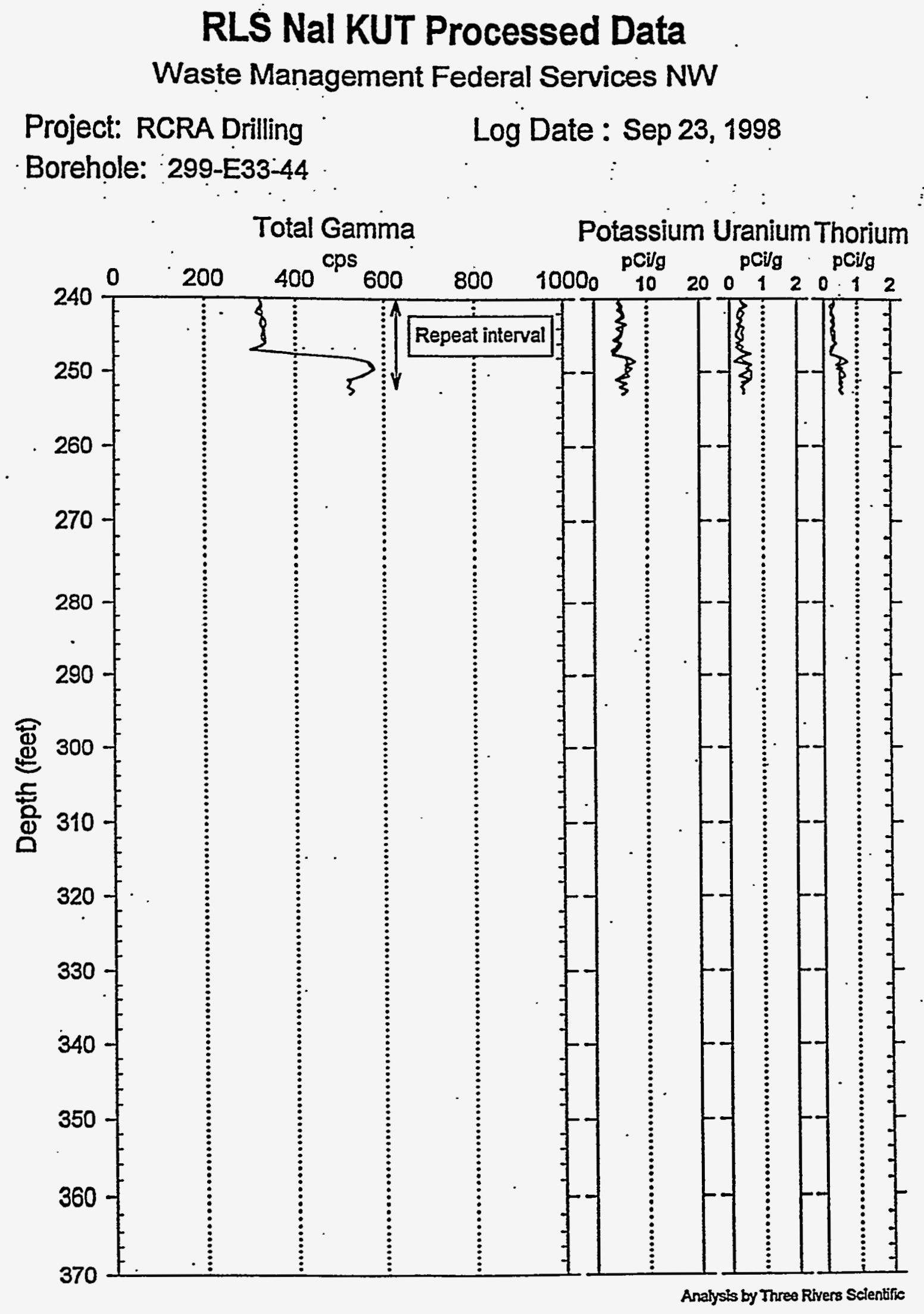




\section{RLS Spectral Gamma Ray Borehole Survey Acceptance QA Processing}

Project: · RCRS Drilling Borehole: 299-E33-44
Log Date: SEp 23, 1998 Compare Main Log and Repeat

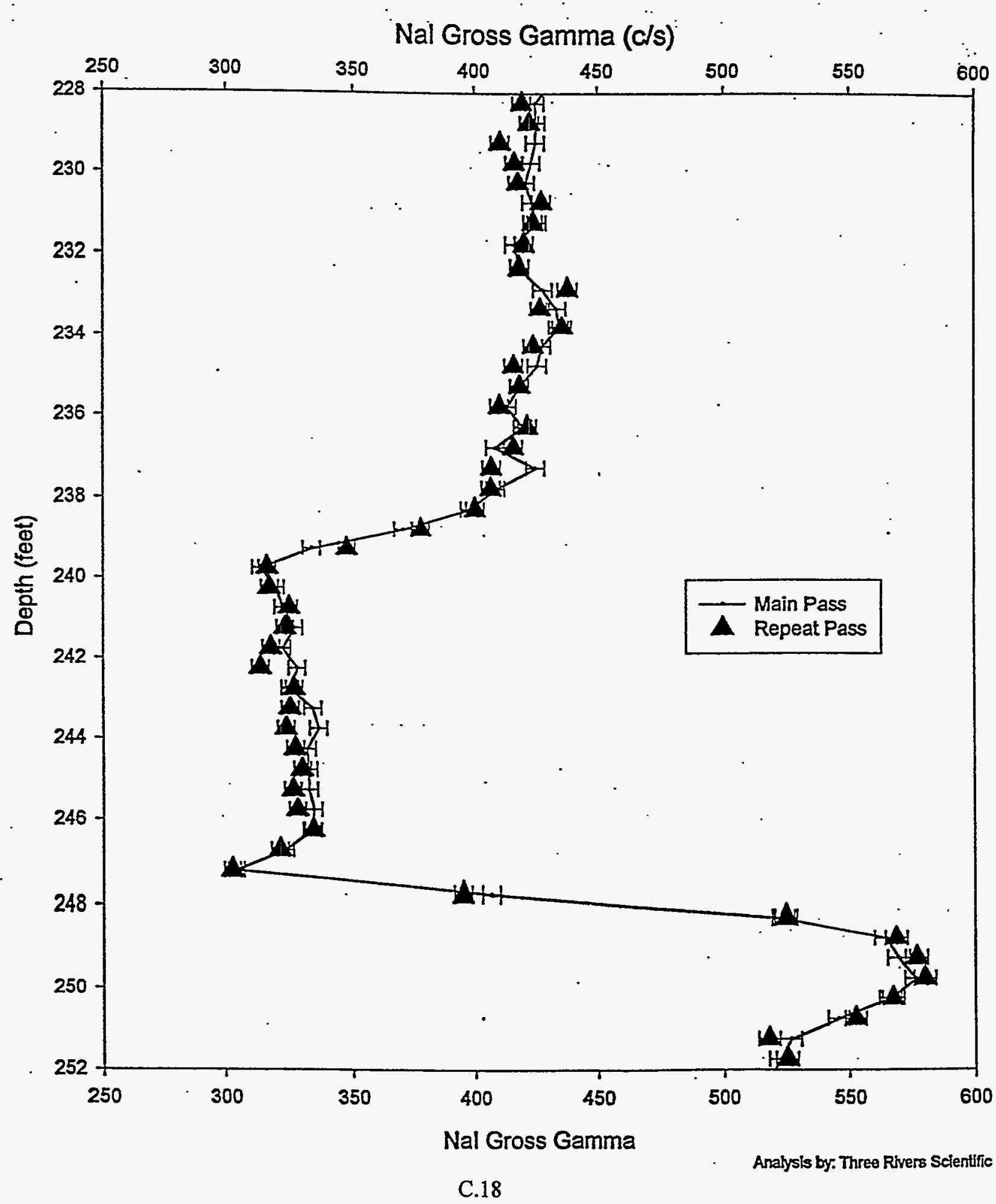




\section{RLS Spectral Gamma Ray Borehole Survey \\ Waste Management Federal Services NW \\ Log Analysis Summary Report}

Project: RCRADrilling

Iog Type: NaI Spectral Gamma Ray

Well ID:

Log Dates: Sep 23, 1998

\section{Basic Response:}

Total gamma is, in general, a response of formation lithology, except for the near surface peak at 2 feet. Cs-137 is identified with the HPGe logging system in the peak at 2 feet. The NaI detector logging system is only calibrated for the natural radionuclides and not man-made radionuclides.

The potassium, uranium and thorium concentrations are normal for Hanford formations.

\section{Repeat Interval:}

The repeat interval, 228 to 252 feet, agrees with the main log within acceptable limits (refer to the Acceptance . QA Processing plot).

\section{Environmental Corrections:}

The KंUT concentrations have been corrected for casing attenuation over the entire well. No casing correction was applied to the total gamma due to Compton downscatter interference. Water correction was applied over the intervals where water is present in the borehole. 


\section{RLS Nal Special Processed Data Waste Management Federal Services NW}

Project: RCRA Drilling Borehole: 299-E33-44
Log Date : Sep 23, 1998

KUT Processed for Counts/Second

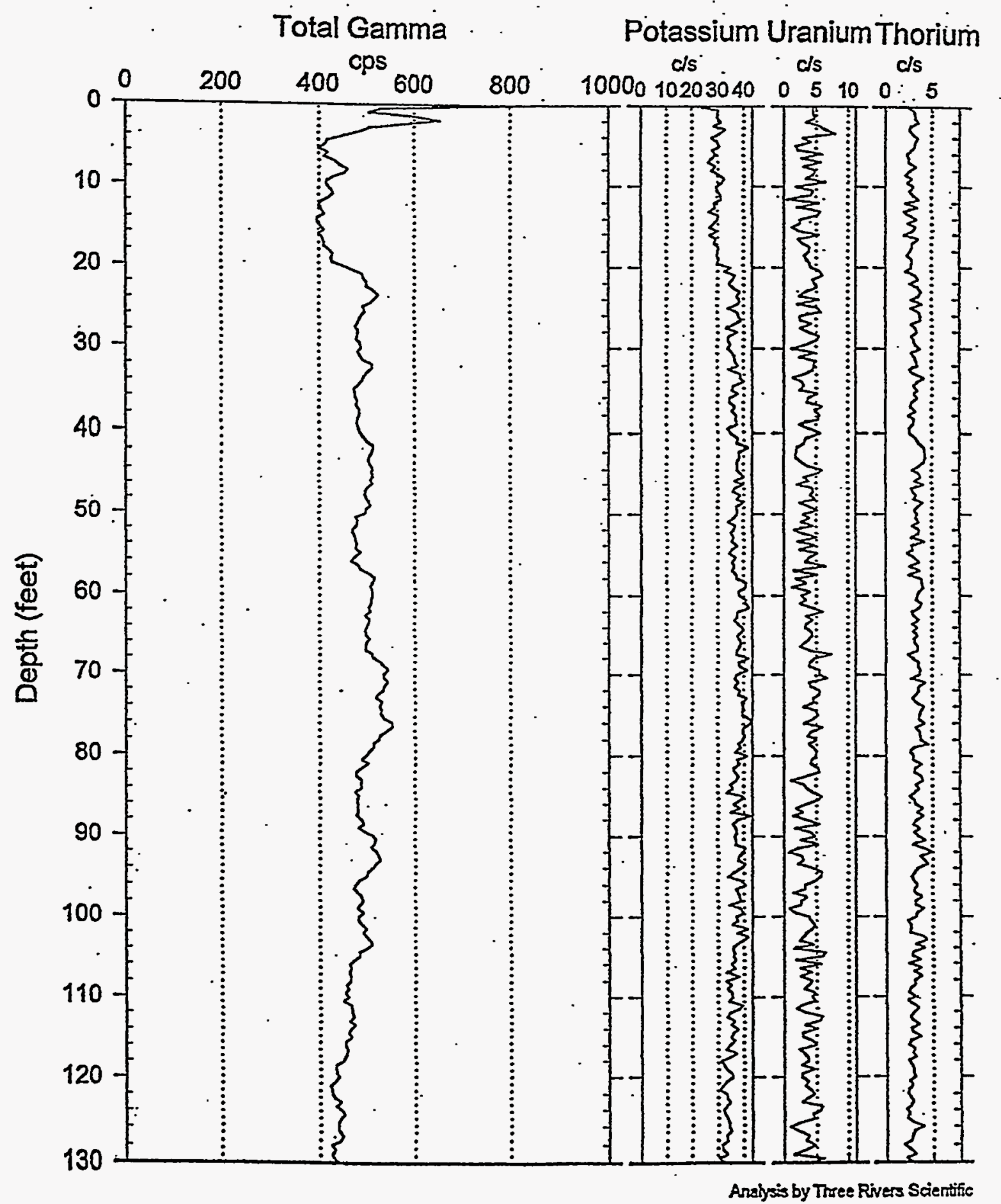




\section{RLS Nal Special Processed Data}

Waste Management Federal Services NW

\section{Project: RCRA Drilling}

Borehole: 299-E33-44
Log Date: Sep 23, 1998

KUT Processed for Counts/Second

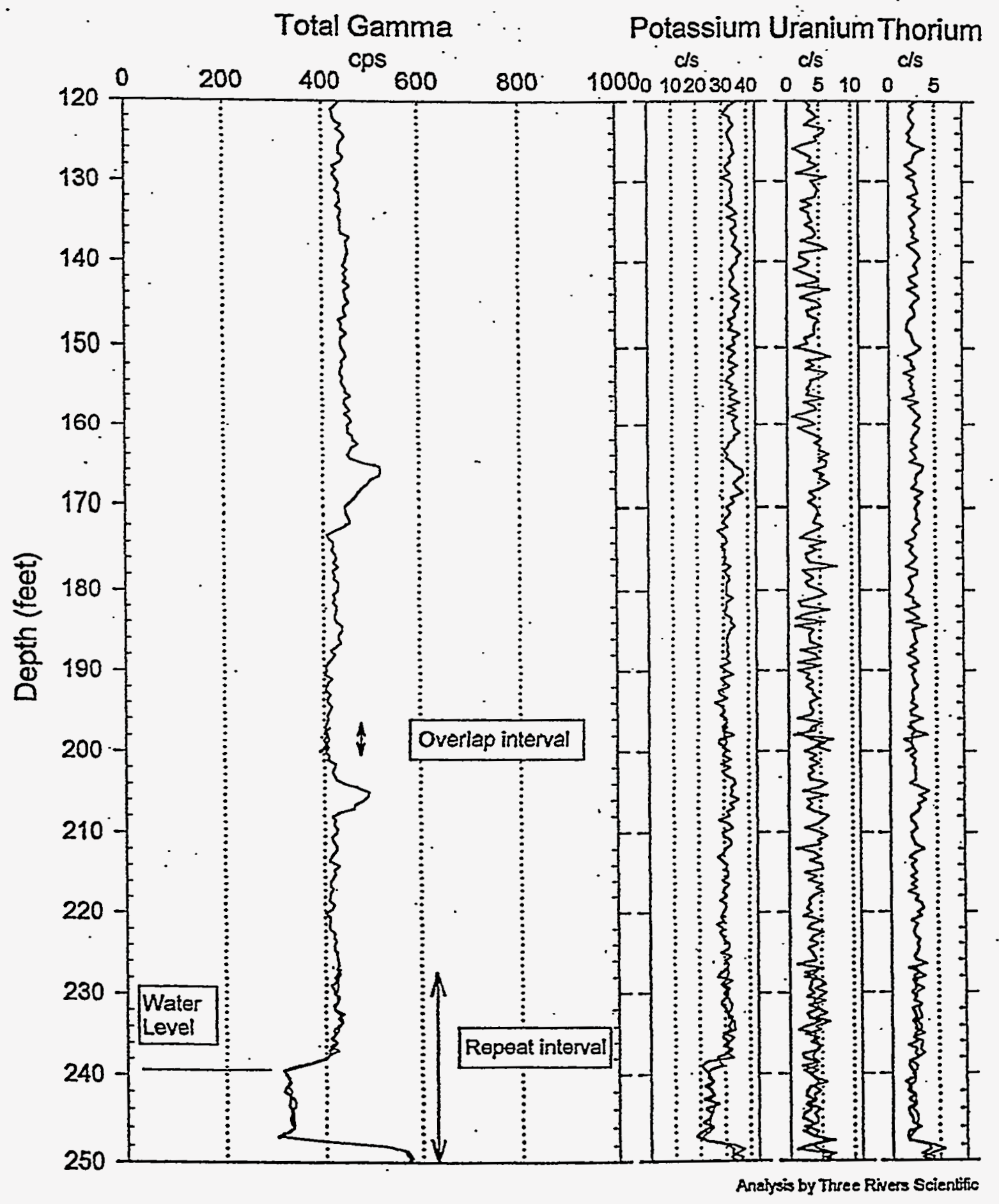




\section{RLS Nal Special Processed Data}

\section{Waste Management Federal Services NW}

Project: RCRA Drilling

Borehole: 299-E33-44
Log Date : Sep 23, 1998

KUT Processed for Counts/Second

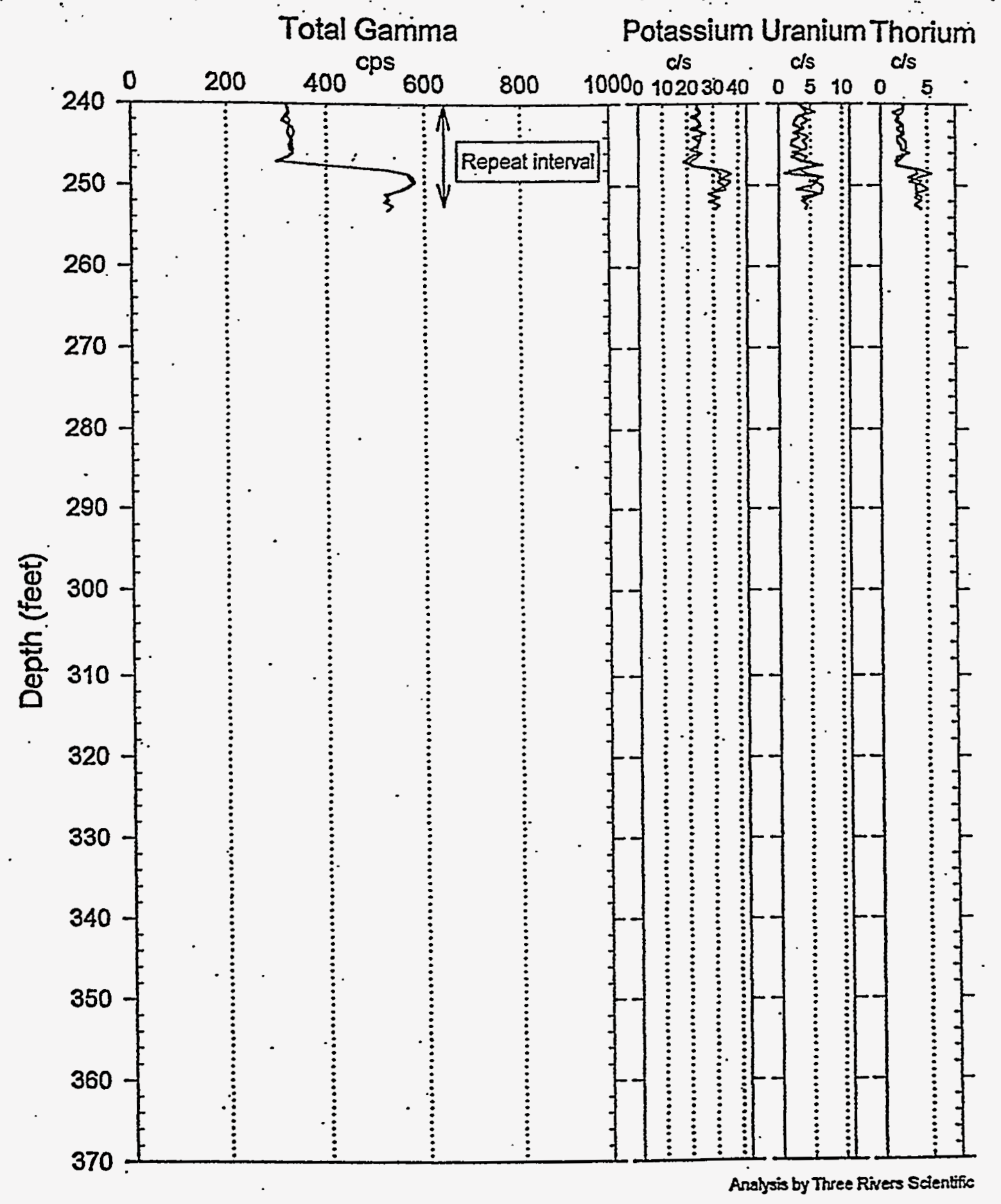




\section{RLS Spectral Gamma Ray Borehole Survey Acceptance QA Processing}

Project: RCRS Drilling Borehole: 299-E33-44
Log Date: SEp 23, 1998

Compare Main Log and Repeat

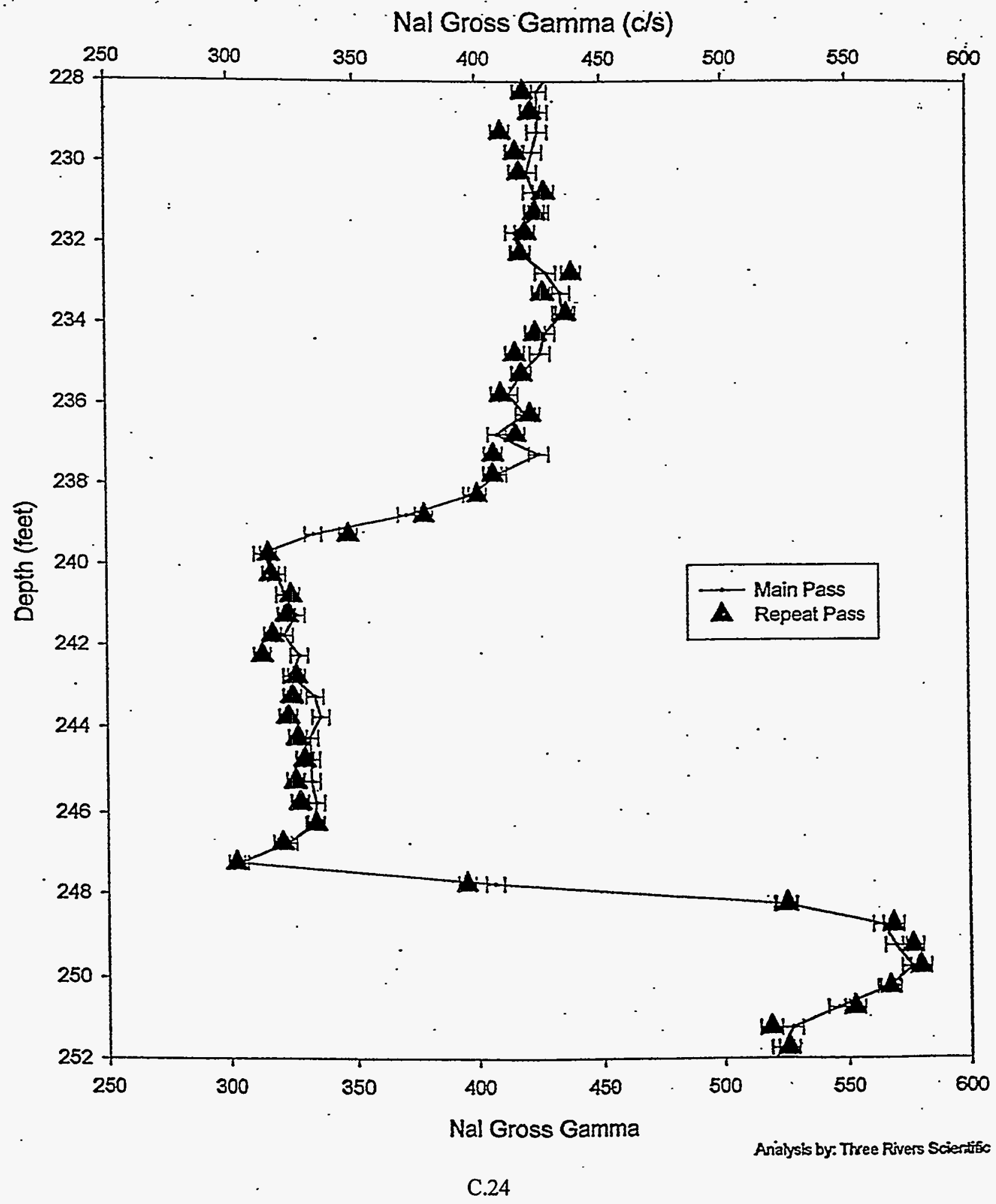




\section{RLS Spectral Gamma Ray Borehole Survey Special Counts/Second Processing \\ Waste Management Federal Services NW}

\section{Log Analysis Summary Report}

$\begin{array}{lllr}\text { Project: } & \text { RCRA Drilling } & \text { Well D: } & \text { 299-E33-44 } \\ \text { Log Type: } & \text { NaI Spectral Gamma Ray } & \text { Log Dates: } & \text { Sep 23, } 1998 \\ & & & \end{array}$

\section{Basic Response:}

Total gamma is, in general, a response of formation lithology, except for the near surface peak at 2 feet. Cs-137 is identified with the HPGe logging system in the peak at 2 feet. The Nal detector logging system is only calibrated for the natural radionuclides and not man-made radionuclides.

The potassium, uranium and thorium concentrations are not plotted, but the equilivent count per second are plotted. These count rates bave been casing and water corrected.

\section{Repeat Interval:}

The repeat interval, 228 to 252 feet, agrees with the main log within acceptable limits (refer to the Acceptance QA Processing plot).

\section{Environmental Corrections:}

The KUT count rates have been corrected for casing attenuation over the entire well. No casing correction was applied to the total gamma due to Compton downscatter interference. Water correction was applied to the KUT count rates over the intervals where water is present in the borehole.

There is no relative difference between the units of count rates and concentrations. This fact is due to the scaling factor relationship between the casing corrected count rates and the concentrations. The scales for the KUT plots have been adjusted to produce an exact overlay of the two sets of plots. The scales are specifically.

K: $0-43.3 \mathrm{c} / \mathrm{s}$

U: $0-11.3 \mathrm{ds}$

Th: $0-7.94 \mathrm{ds}$ 


\section{Methodology for Deriving KUT Net Count Rates for Nal Spectral Data \\ : Russel Randall, $\mathrm{PhD}$ \\ December 14, 1998}

\section{Background}

NaI spectral data collected by Waste Management Northwest (WM-NW) is presently processed to produce $\mathrm{K}, \mathrm{U}, \& \mathrm{Th}$ concentrations. The calibration ${ }^{2}$ produces a set of coefficients that are nsed to convert the count rate observed in selected energy windows to the $K, U, \&$ Th concentrations. This 3 by 3 matrix of coefficients does not produce the net effective $K, U, \&$ Th count rates.

A request was made to produce coumt rates for each of the radionuclides $K, U, \&$ Th from NaI logging data. In order to meet this request, a different calibration techrique is required, and then application of resulting coefficients to the log data yields net count rates. This document provides the description of the methodology used to derive the coefficients that can be applied to log data in order to generate the net count rates for $K, U, \&$ Th as a function of depth.

\section{Basic Spectral Response}

The detected signal for $\mathrm{NaI}$ logging data is observed events as a function of the energy of the pulse event detected. Figure 1 contains a representative spectra from the $K$ model and the $U$

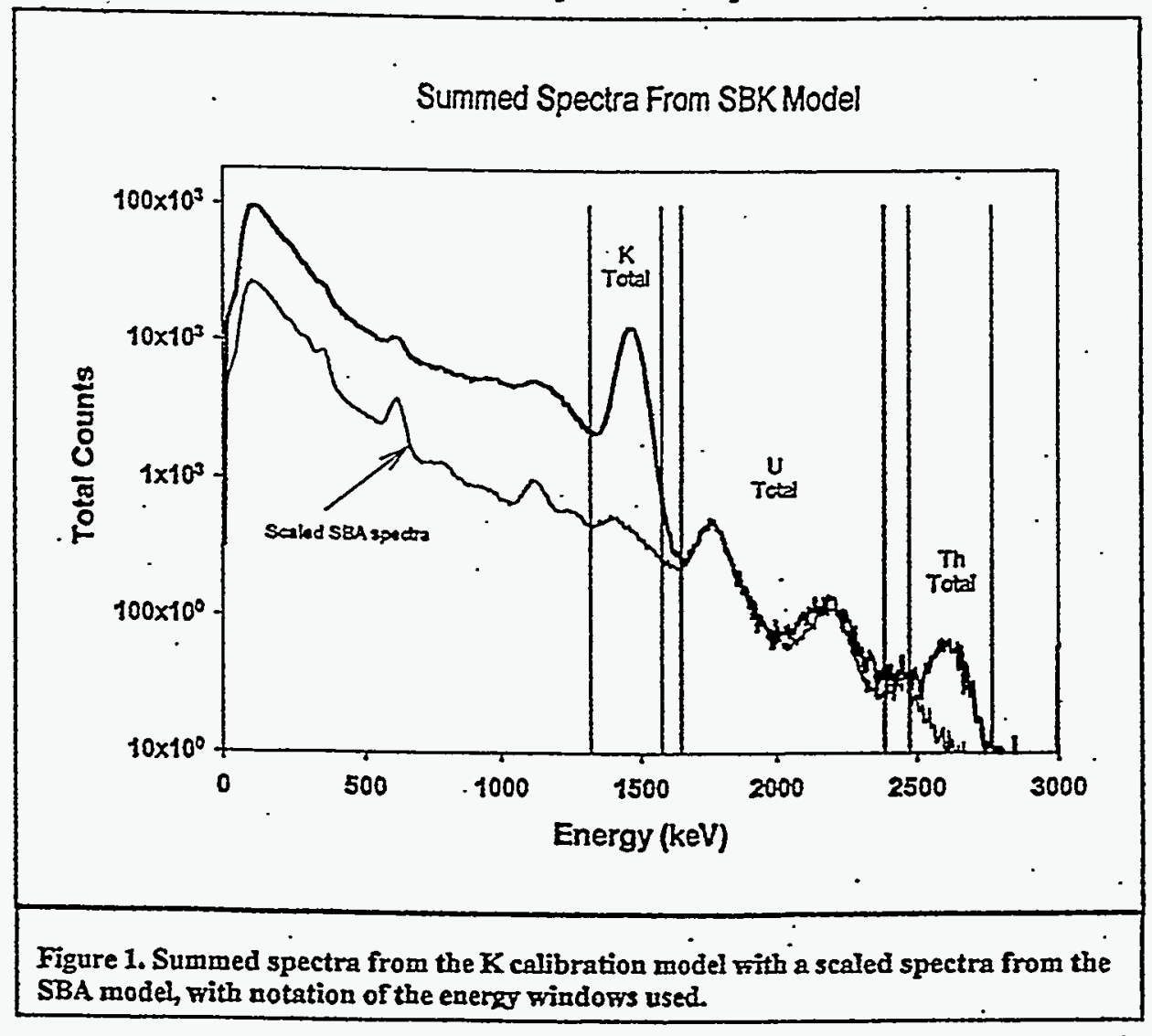


model at the Hanford site. The inherent energy resolution of the NaI crystal becomes an issue when there are cither multiple radionuclides or any given radionuclide has multiple photo peaks from several gamma rays. Both natural nranium, $U$, and thorium, $T$, have many photo peaks that are not clearly resolved. However, tinere are analysis techniques that can be applied to deconvolve the response of $\mathrm{K}, \mathrm{U}, \&$ Th gamma rays from a minimum of three observed counit rates from three energy windows (refer to fig 1 for the windows used by WM-NW analysis procedures).

Consider the energy window labeled K-Total. This energy window covers all the observed gamma events between 1320 and $1575 \mathrm{keV}$. Any observed gamma event recorded in this window can be from one of the following:

1. K photo peak

2. K Compton (on the lower portion of the window)

3. U photo peaks

4. U Compton

5. Th photo peaks

6. Th Compton

All of these 6 sources of observed gamma rays can be lumped into three terms; all event caused by the presence of $\mathrm{K}$, all events caused by the presence of $U$, and likewise, Th All events cause by $\mathrm{K}$ is defined as the net $\mathrm{K}$ count rate component of the events recorded in the total window. All events observed in the window due to the presence of $K$ is proportional to the $K$ concentrations, thus,

$$
K=\alpha_{K} \cdot C_{K}^{n e t} \quad \text { Eq. } 1
$$

where, $\mathrm{K}$ is the potasium concentration in $\mathrm{pCi} / \mathrm{g}, \alpha_{\mathrm{K}}$ is a calibration coefficient, and $\mathrm{C}_{\mathrm{X}}^{\text {ret }}$ is the net count rate in the $K$-total window that is due to the presence of $K$ On the basis of the above discussion for the sources of the recorded events, the net count rate from $K$ can be described by the following:

$$
C_{K}^{n e t}=C_{K}-a_{K U} \cdot U-a_{K T} \cdot T
$$

where, $C_{K}$ is the total observed count rate in the $\mathrm{K}$-total window, $U$ is the uranium concentration in the formation, $a_{k v}$ is a calibration coefficient to be determined, $T$ is the $T h$ concentration in the formation, $a_{k \pi}$ is a calibration coefficient to be determined. Substitution of this expression into the previous sesults in:

$$
\begin{aligned}
& K=\alpha_{K} \cdot C_{K}-\beta_{K U} \cdot U-\beta_{K T} \cdot T \\
& \beta_{K U} \equiv \alpha_{K} \cdot a_{K U} \\
& \beta_{K T} \equiv \alpha_{K} \cdot a_{K T}
\end{aligned}
$$

Therefore, the above equation has three unknowns and can be solved using a minimum of three measurements. The data collected in the SBK, SBA, and SBT models were used tọ solve for these three coefficients.

\section{Data Analysis}

The three model measurements can be used to solve Eq.3 for the coefficients, given the radionuclide concentrations of the models, and the observed K-total count rate of the instrument 
in each model. The derivation for $U$ and Th is similar, except there are only 2 gamma event contributors and therefore, only 2 coefficients to solve for. Therefore, the case of $U$ and $T h$ is . overdetermined, given three model measurements.

The calibration models at Hanford have known concentrations of $K, U, \& \mathrm{Th}^{2}$. Table 1 lists the standards for the models used in this formulation.

Table 1. Standard values for the Hanford calibration models

\begin{tabular}{|c|c|c|c|}
\hline Model & $\mathrm{K}-40(\mathrm{pCi} / \mathrm{g})$ & $\mathrm{U}(\mathrm{Ra}-226)(\mathrm{pCI} / \mathrm{g})$ & Thorinm (pCi/g) \\
\hline SBK & 53.5 & 1.16 & 0.11 \\
\hline SBA & $11^{2}$ & $63.0^{b}$ & $0.66^{2}$ \\
\hline SBT & 10.63 & 10.02 & 58.11 \\
\hline
\end{tabular}

The SBU model has more rigorously defined concentrations of all components over the SBA model, but the detector exceed the high count rate limit for the SBU model. The NaI crystal is designed to be very large and therefore sensitive in order to obtain the most lithologic information with the lowest cost of logging. Likewise, the SBM model exceeds the high count rate limitation for this instrument.

Calibration data were collected in the three models, SBT, SBK, and SBA. Each model was used to record ten spectra of 300 seconds each. Table 2 contains the average total count rate observed for each of the selected energy windows used in the KUT processing. Also included in table 2 are the energy ranges for each of the processing windows.

Table 2. Observed average count rates in the energy windows

\begin{tabular}{|c|c|c|c|}
\hline Model & $\begin{array}{c}\text { K-Total (c/s) } \\
1320-1575 \mathrm{keV}\end{array}$ & $\begin{array}{c}\text { U-Total (c/s) } \\
1650-2390 \mathrm{keV}\end{array}$ & $\begin{array}{c}\text { Th-Total (c/s) } \\
2475-2765 \mathrm{keV}\end{array}$ \\
\hline SBK & 83.58 & 6.98 & 0.637 \\
\hline SBA & 335.9 & 361.3 & 11.44 \\
\hline SBT & 297.2 & 563.4 & 231.7 \\
\hline
\end{tabular}

Appendix A contains the details of the calculations for the coefficients. The only coefficients of application are the coefficients of eq. 1 for each of the radionuclides, $K, U, \&$ Th. Table 3 contains the resulting values as determined from the calibration data and simultaneous solutions.

Table 3. Resulting conversion coefficients for the effective net count rates

\begin{tabular}{|c|c|}
\hline Radionuclide & Calibration Factor (pCi/g)/(c/s) \\
\hline K-40 & 0.692 \\
\hline Uranium (Ra-226) & 0.176 \\
\hline Thorium & 0.252 \\
\hline
\end{tabular}

\section{Verification}

It is possible to arrive at an independent estimate for the $\mathrm{K}$ net count rate since this radionuclide. has only one emitted gamma ray. This fact can be used to derive the net photo peak count rate by fitting a gaussian to observed spectra. This estimate will be somewhat less than the value derived 
and listed in table 3 , since only the photo peak net count rate can be measured using this technique, and the derived coefficients are a result of photo peak intensity and Compton background intensity due to the presence of $K$.

A gaussian plus linear background fit was performed for the $K$-model summed spectra for the dominart $1461 \mathrm{keV}$ gamma ray, refer to fig 1. Appendix B contains the details of this peak . fitting calculation. The resulting count rate for this photo peak intensity above background is $65.2 \mathrm{c} / \mathrm{s}$. This count rate is $14 \%$ lower than that derived on the basis of the coefficient listed in table 3. As can be seen in fig 1, some of the count recorded in the K-total window are due to both $\mathrm{K}-40$ and its Compton down scatter. Thus the $14 \%$ lower figure for just the photo peak is reasonable and substantiates the total "niet" count rates that can be arrived at by using the coefficients in table 3 .

\section{Results}

The methods have been described covering the derivation of the scaling factors that relate the net effective count rates for $K, U, \&$ Th from the elemental concentrations. This derivation has been verified using a peak fitting for the single photo peak of $\mathrm{K}-40$ in the SBK calibration model.

The application of this derivation to the production of $\log$ plots in "net" effective KUT count rates is then straight forward. If the normal log analysis has been performed, then the elemental concentrations have been casing and/or water level corrected and the count rates are simply a result of scaling the concentrations by division of the coefficients listed in table 3 . Note if the normal log analysis has not been performed, then apply this normal analysis and then proceed with the scaling to produce the net effective count rates. It must be pointed out that since the scaling factor is a constant, then there is no relative difference between the elemental concentrations and the net effective count rates.

\section{References}

1. R. Randall and D. Stromswold, "Procedures for Calibrating Scintillation Gamma-Ray- Well Logging Tools Using Hanford Formation Models, WHC-SD-EN-TI-293, Rev. 0, Westinghouse Hanford Company, Richland, WA 1995.

2. W.D. Steele and D.C. George, "Field Calibration Facilities for Environmental Measurement of Radium, Thorium and Potassium," US. Dept. of Energy report GJ/TMY C-01 ( $2^{\text {nd }}$ ed) UC70A, Bendix Fiela Eng., Grand Junction, CO, 1986. 


\section{Appendix A Computations for Coefficients}

Finding coefficient for Th coversion from count ratesito concentration...

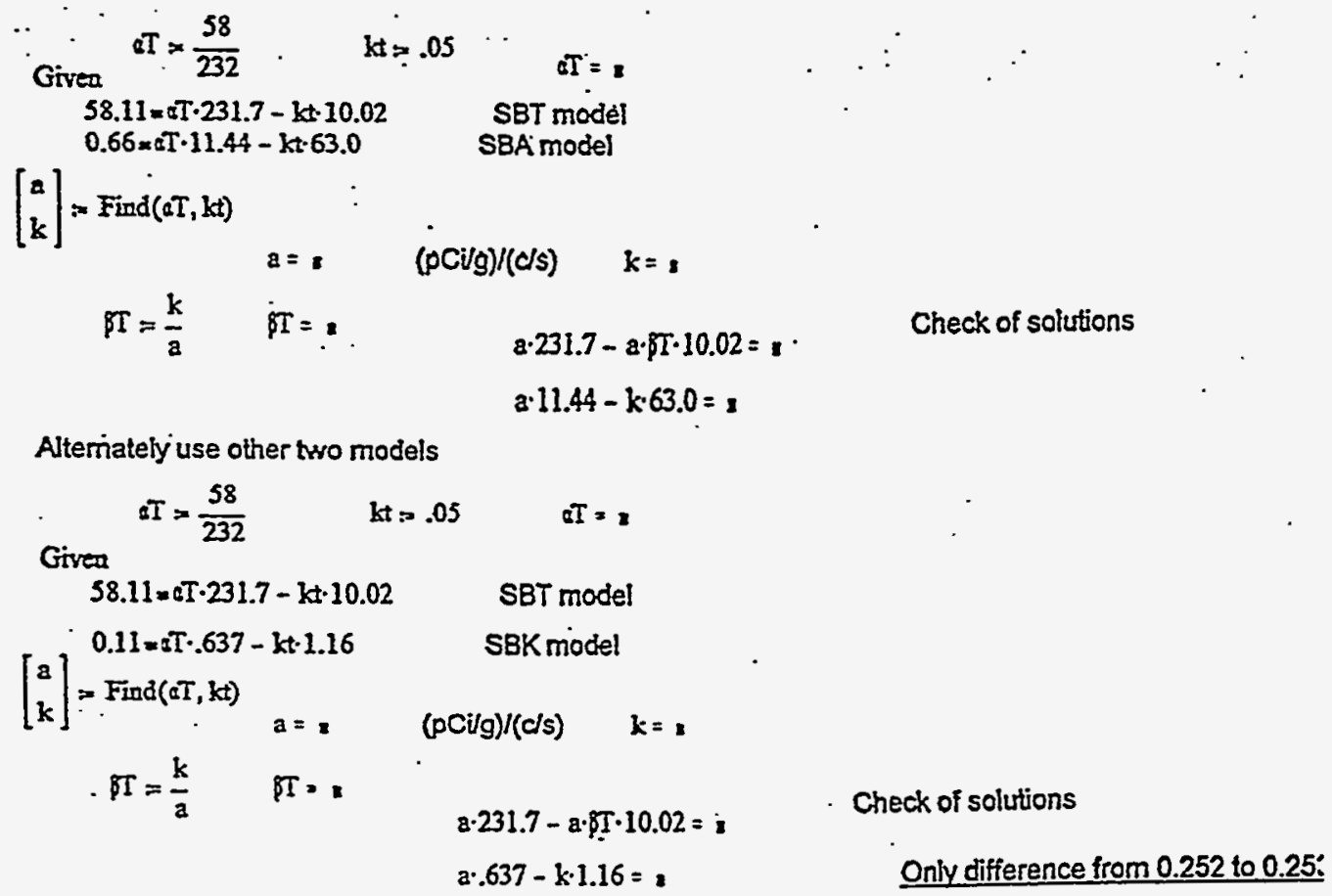

Alteriately use other two models

Process for U coefficient

$$
\begin{aligned}
& U=\alpha U^{*} G u-k 1^{*} K-k_{2}^{*} T \quad \text { for each model }
\end{aligned}
$$

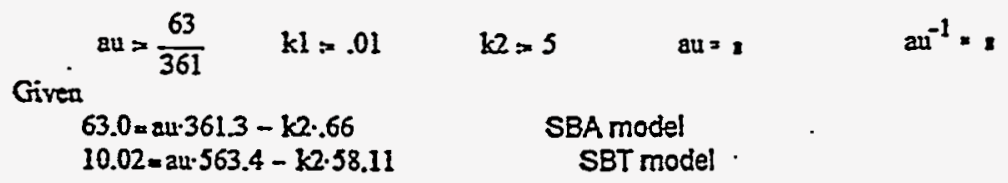

$$
\begin{aligned}
& {\left[\begin{array}{l}
a \\
d
\end{array}\right]=\text { Find }(a w, k 2)} \\
& \mathbf{a}=\mathbf{x} \\
& \text { Checking } \\
& \mathrm{a} \cdot 361.3-\mathrm{d} \cdot .66=\text {, } \\
& \text { a.563.4-d.58.11 = } \\
& \text { a.6.98 - d. } .11=
\end{aligned}
$$




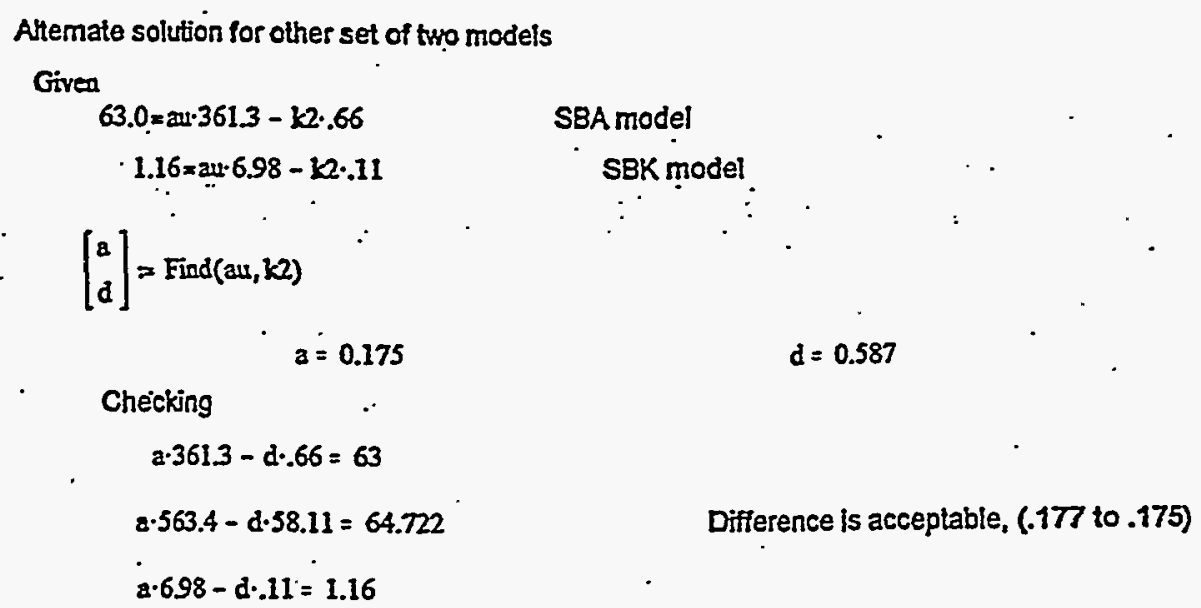

$$
\mathrm{d}=0.587
$$

Difference is acceptable, (.177 to .175)

Process for $K$ coefficient

$\begin{array}{llc}K=\alpha k^{*} G k-k 1^{*} U-k 2^{*} T & \text { for each model } \\ 2 k=\frac{53 \cdot 2}{83} & k 1=1.5 & k 2=2 \quad . k=0.128\end{array}$

Given

$11=2 k \cdot 335.9-k 1 \cdot 63.0-k 2 \cdot .66 \quad$ SBA model

$10.63 \times$ alk:297.2 $-k 1 \cdot 10.02-k 2 \cdot 58.13 \quad$ SBT model

$53.5=a k \cdot 83.58-k 1 \cdot 1.16-k 2 \cdot .11, \quad S 8 K$ model

$\left[\begin{array}{l}a \\ c \\ d\end{array}\right]=$ Find $(a k, k 1, k 2)$

$a=0.692 \quad c=3.487 \quad d=2.756$


Appendix B Calculations for peak and background fitting

K spectral stripping...

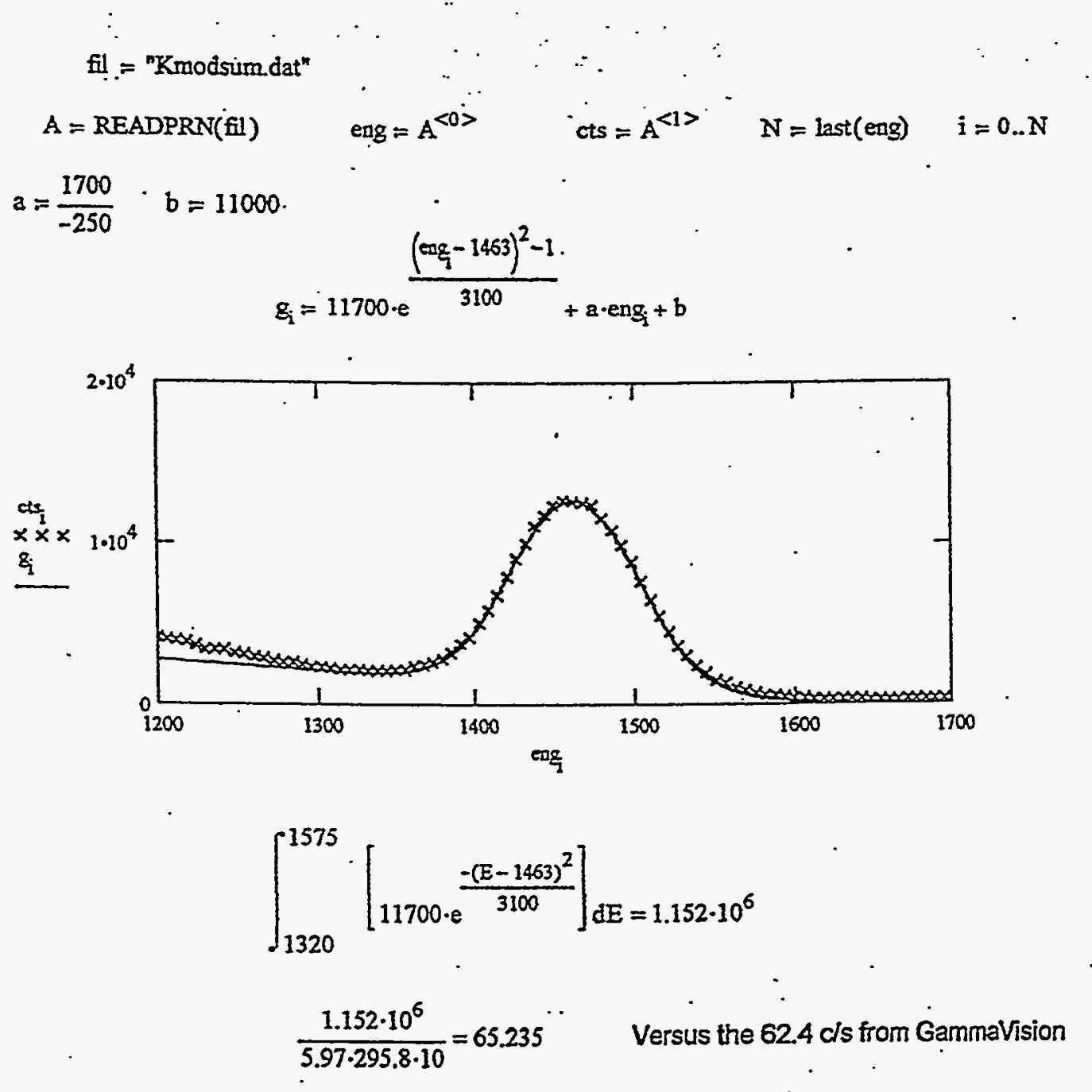

When I fit the exponential only the total curve, I get $14 \%$ higher count rate due mostly t. the Compton background on the low energy side. Thus the true stripped nel is low by: least $15 \%$ from the $K$ contribution in the energy window used. 


\section{RLS Moisture Borehole Survey \\ Waste Management Federal Services NW}

\section{Log Header}

Project: RCRA Drilling

Well: 299-E33-44

Log Type: Moisture

Borehole Information

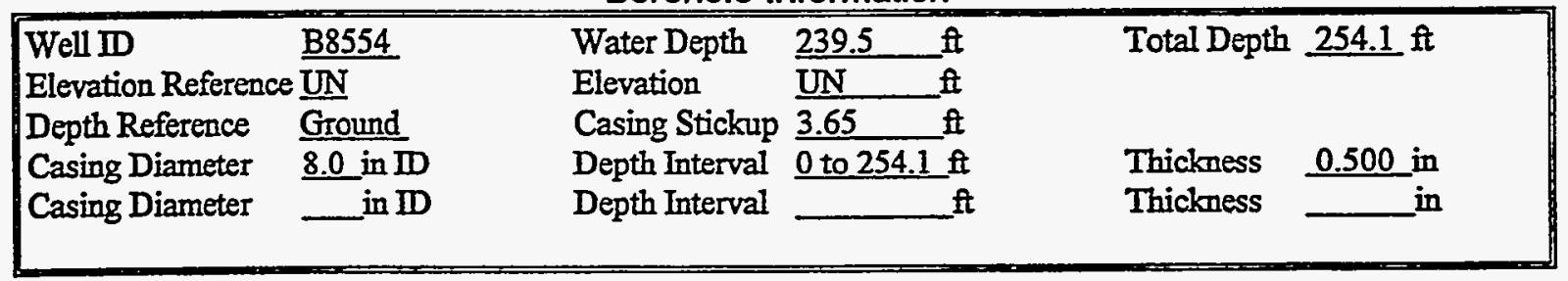

Logging Information

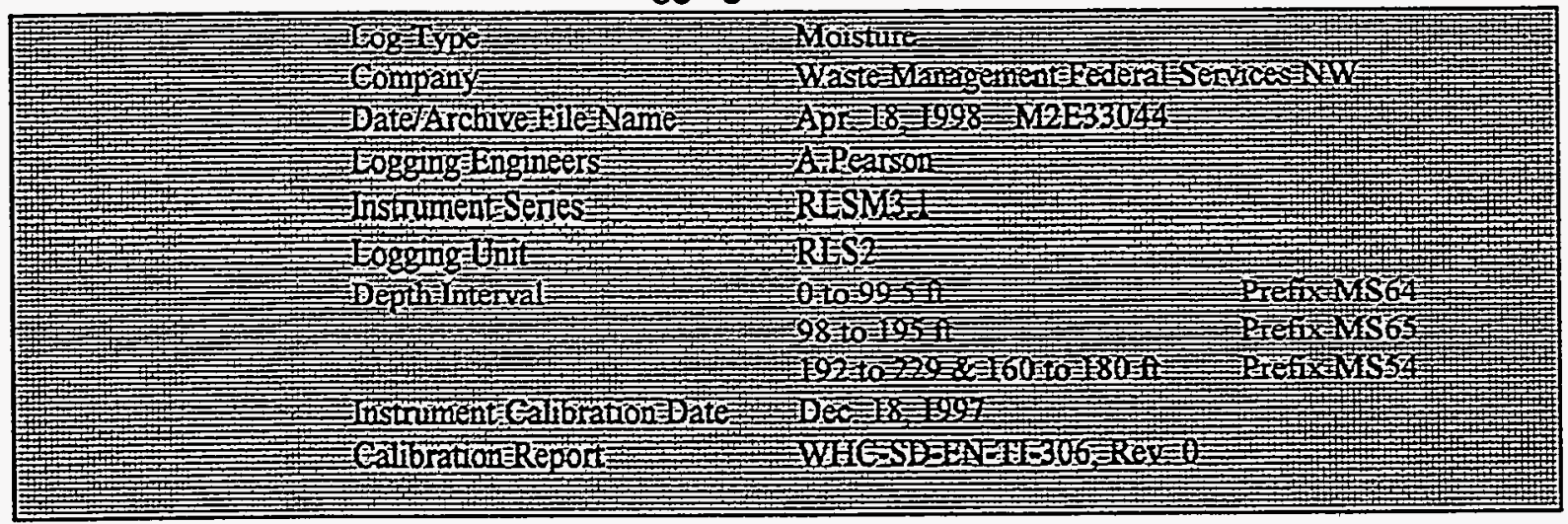

Analysis Information

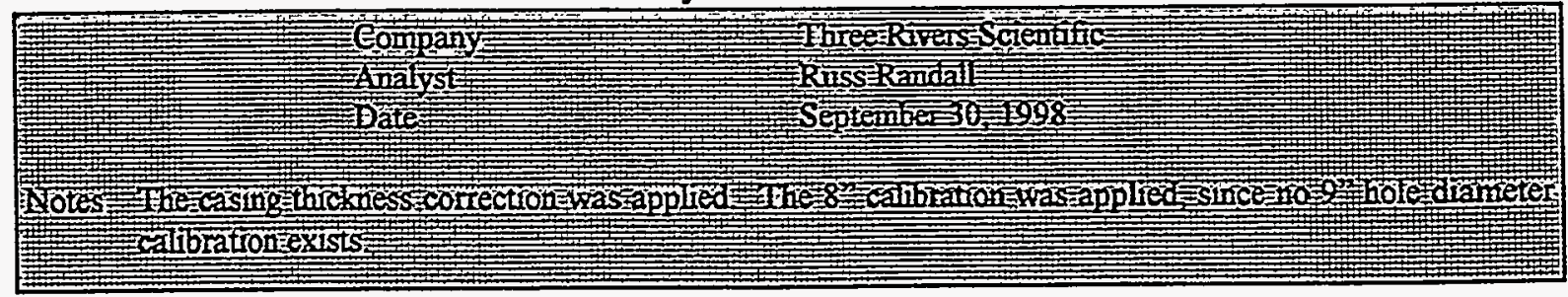




\section{RLS Moisture Processed Log Data.}

\section{Waste Management Federal Services NW}

\section{Project: RCRA Drilling}

Borehole: 299-E33-44

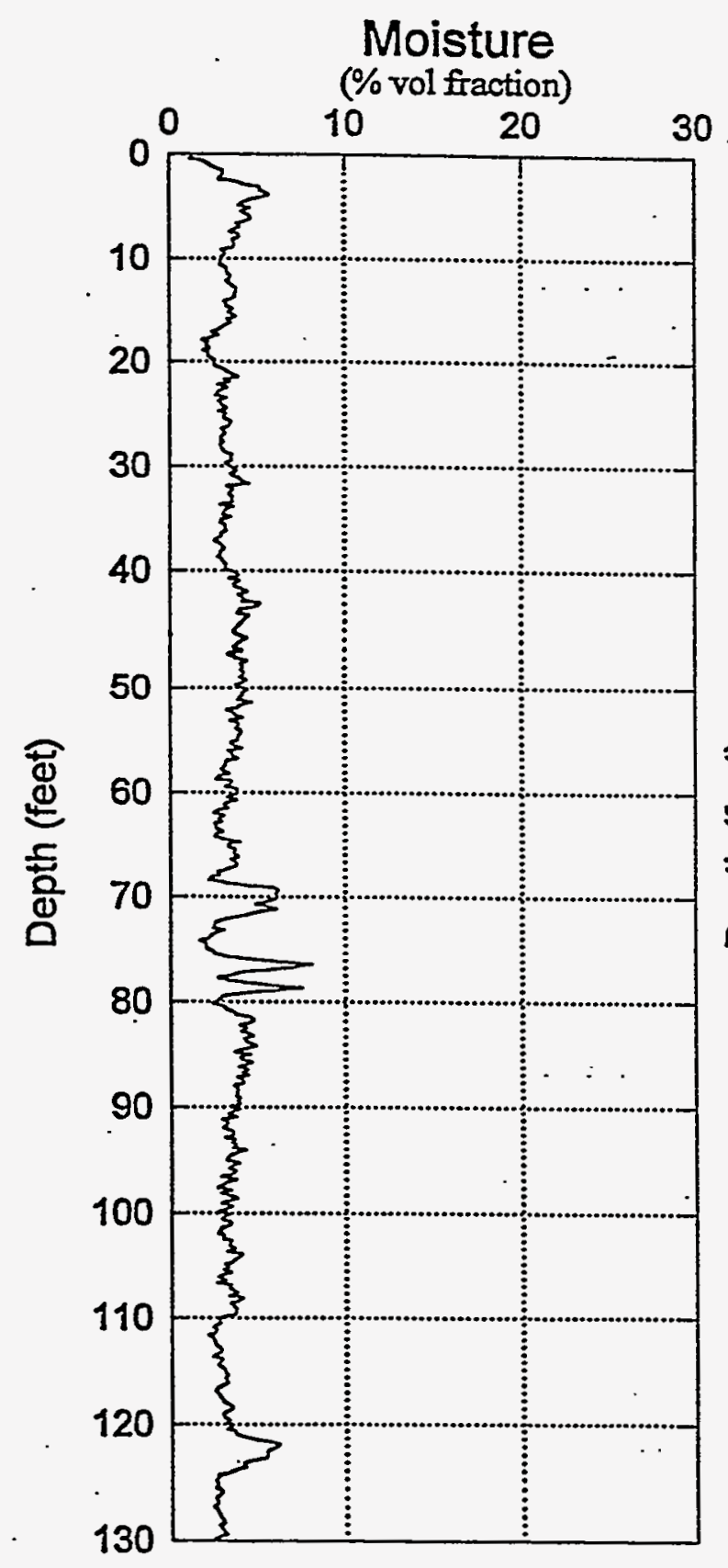

Analysis by. Three Rivers Scientific
Log Date Sep 23, 1998

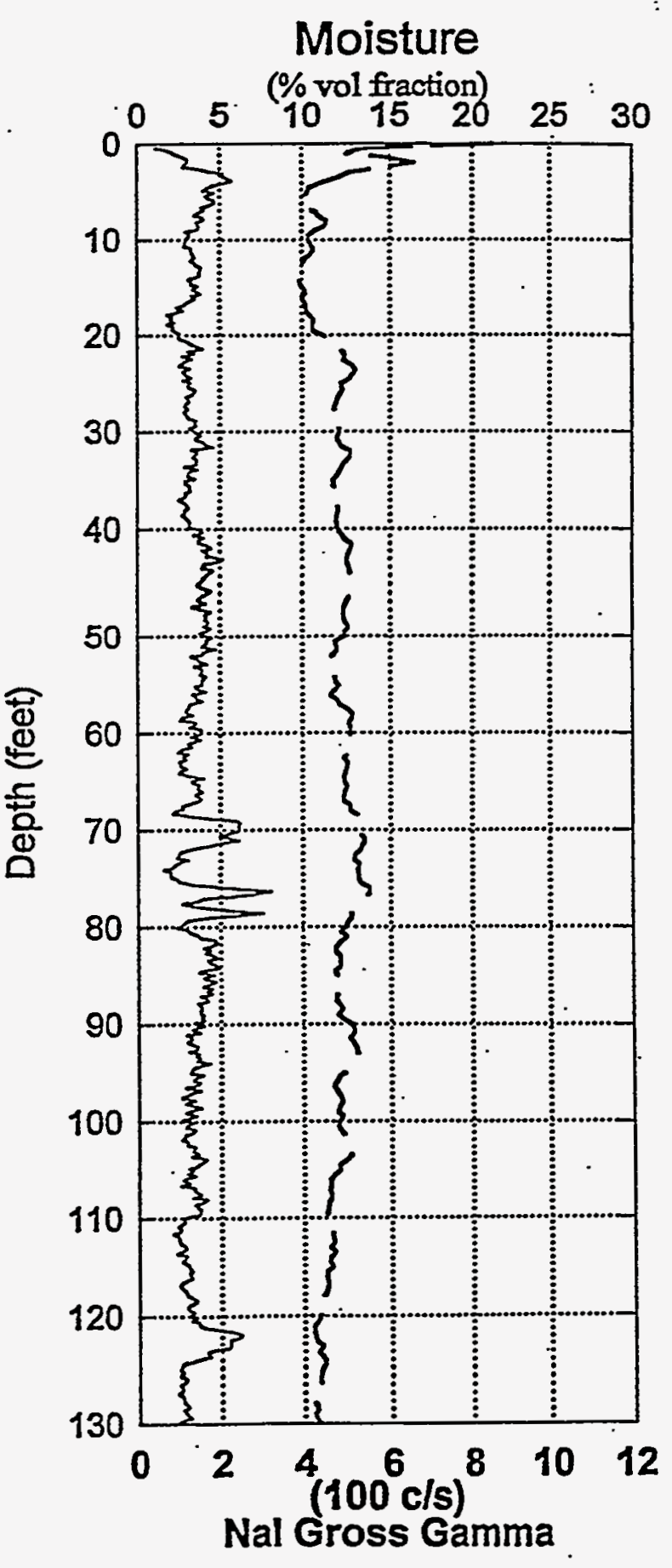




\section{RLS Moisture Processed Log Data Waste Management Federal Services NW}

\section{Project: RCRA Drilling}

Borehole: 299-E33-44

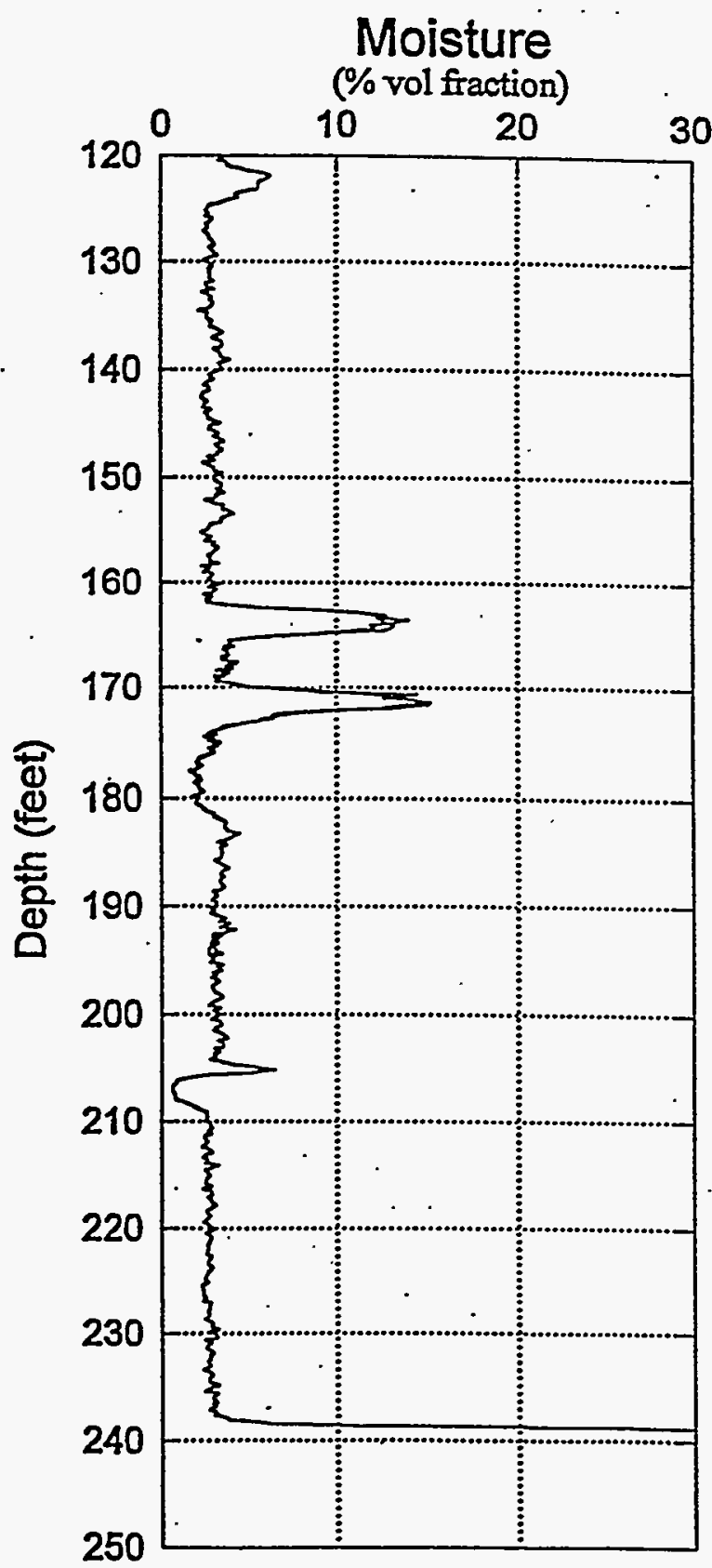

Analysts by. Three Rivers Scientific
Log Date. Sep 23, 1998

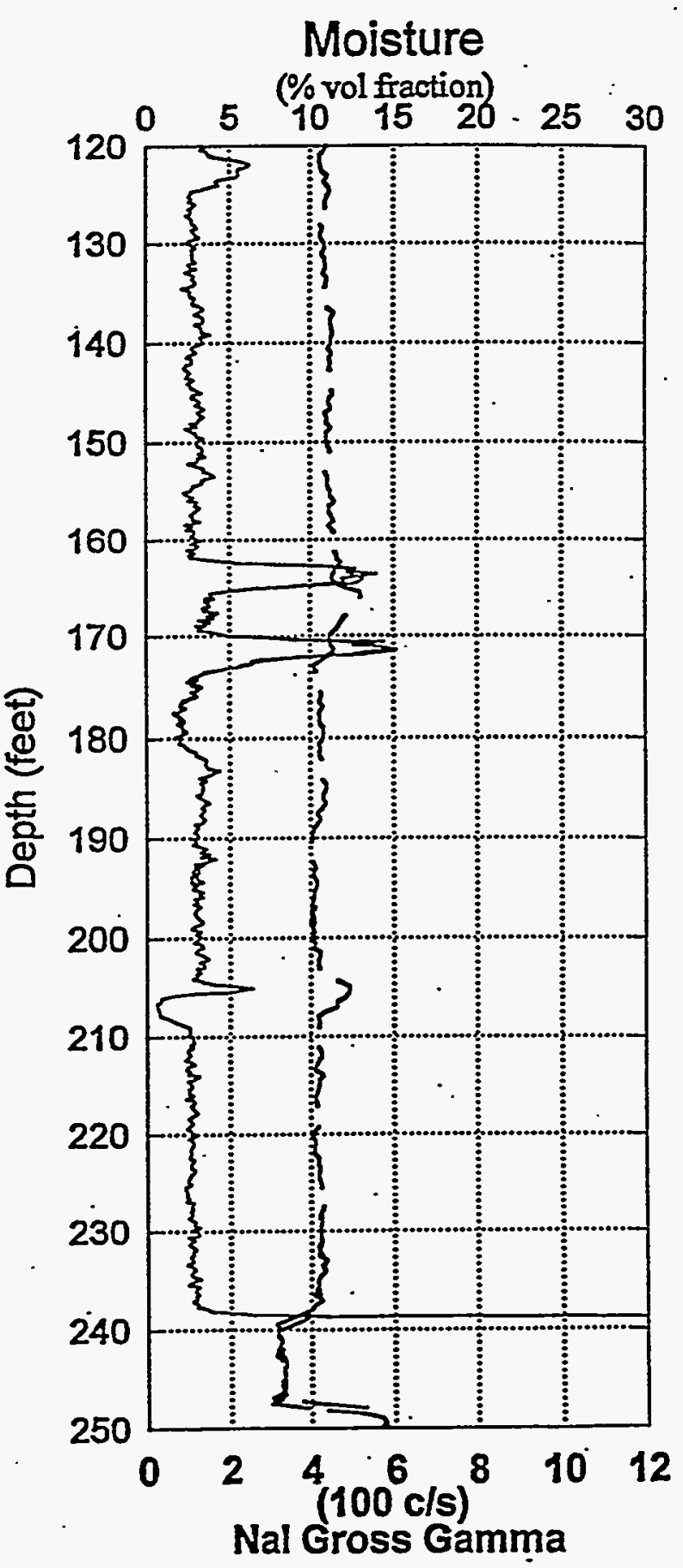




\section{RLS Moisture Borehole Survey \\ Acceptance QA Processing}

Project: RCRA Drilling

Borehole: 299-E33-44
Log Date: Sep 23, 1998

Compare Main Log and Repeat

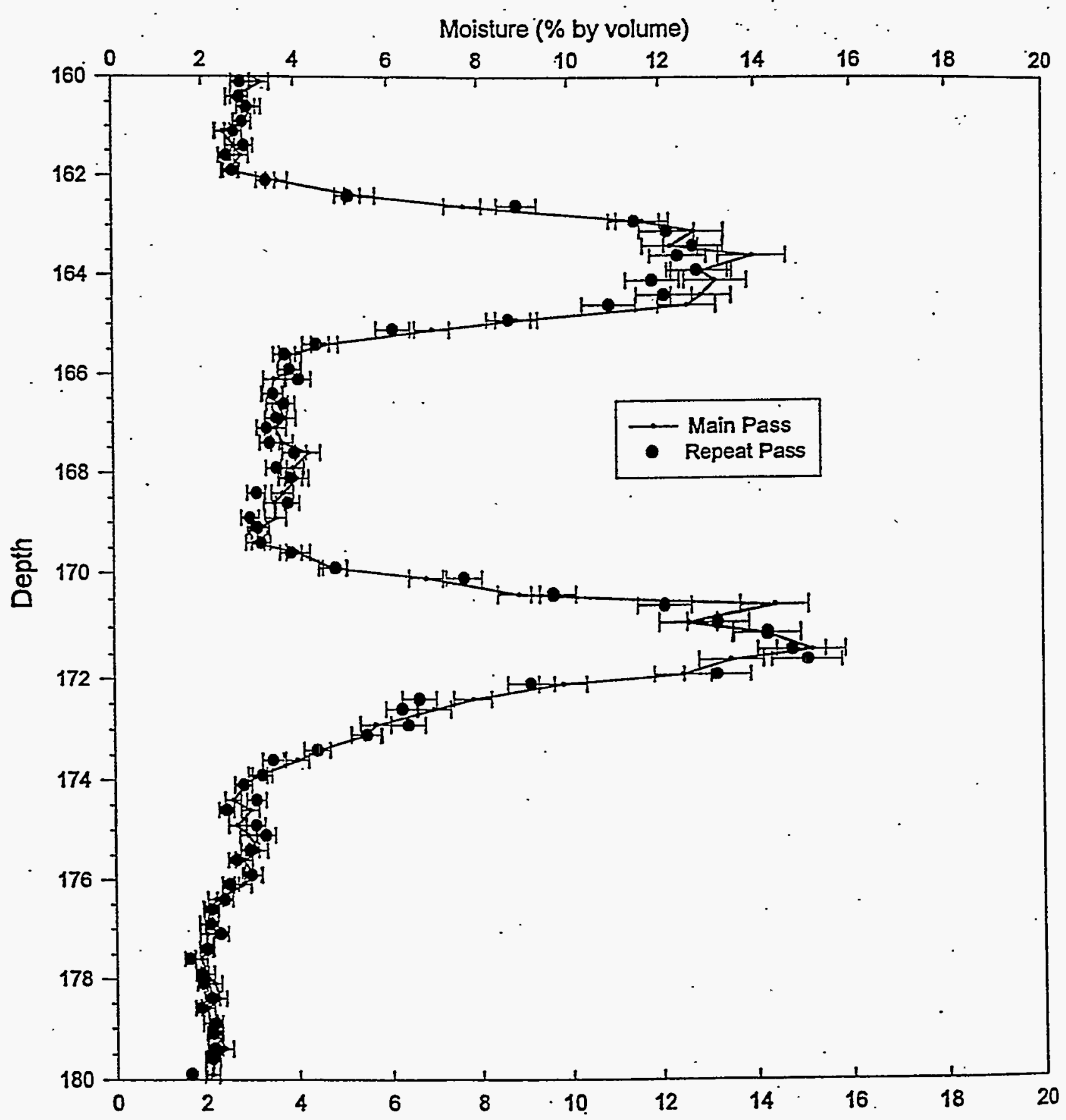




\title{
RLS Moisture Borehole Survey \\ Waste Management Federal Services NW
}

\section{Log Analysis Summary Report}

\author{
Project: RCRADrilling \\ Well ID: \\ 299-E33-44 \\ Log Type: Moisture \\ Log Dates: Sep 23, 1998
}

\section{General Notes:}

At these low moisture values for the earth surround the borehole, other parameters such as void space and formation density affect the instrument readings more than moisture, since so little moisture is present. There are tro elevated moisture zones at 164 and 172 feet. Also, the very low reading zone at 207 feet is most likely the presence of a void space behind the casing.

There does appear a small correlation between gross gamma ray (i.e. lithology) and the moistrre.

System Performance Verification: The pre- and post-log verification was performed using instrument carrier. The pre-log reading is $4 \%$ lower than the post-log reading, well within tolerance.

Repeat Interval: The repeat interval, 160 to 180 fet, agrees with the main log within acceptable limits (refer to the Acoptance QA Processing plot).

Environmental Corrections: The casing thickness, correction has been applied. A density correction was not applied.

The borehole diameter is a nominal 9 inch value and there is no calibration for this diameter. However, at these low moisture values, an extrapolation would not yield any significant change. 


\section{Distribution}

No. of

Copies

OFFSITE

T. Valero

Washington State Department of Ecology $13154^{\text {th }}$ Avenue

Kennewick, WA 99336

ONSITE

2 DOE Richland Operations Office

M. J. Furman (2)

$\mathrm{H} 0-12$

Bechtel Hanford Company

A. J. Knepp

H0-19

Fluor Daniel Northwest

F. M. Mann

H0-22
No. of

Copies

Lockheed Martin Hanford Company

E. A. Fredenburg

R1-04

18 Pacific Northwest National Laboratory

G. R. Holdren

K6-81

D. G. Horton (2)

K6-81

S. P. Luttrell

$\mathrm{K} 6-96$

L. F. Morasch

K6-86

S. M. Narbutovskih (5) K6-96

F. A. Spane, Jr.

K6-96

Information Release Office (7)

K1-06

Distr.1 